\title{
Inverse scattering transform for the nonlocal nonlinear Schrödinger equation with nonzero boundary conditions
}

Mark J. Ablowitz, Xu-Dan Luo, and Ziad H. Musslimani

Citation: Journal of Mathematical Physics 59, 011501 (2018); doi: 10.1063/1.5018294

View online: https://doi.org/10.1063/1.5018294

View Table of Contents: http://aip.scitation.org/toc/jmp/59/1

Published by the American Institute of Physics

\section{Articles you may be interested in}

Nonlocal nonlinear Schrödinger equations and their soliton solutions

Journal of Mathematical Physics 59, 051501 (2018); 10.1063/1.4997835

Almost global existence for the nonlinear Klein-Gordon equation in the nonrelativistic limit Journal of Mathematical Physics 59, 011502 (2018); 10.1063/1.4994969

Generalized delta functions and their use in quantum optics

Journal of Mathematical Physics 59, 012102 (2018); 10.1063/1.4985938

Integrability of the one dimensional Schrödinger equation

Journal of Mathematical Physics 59, 022105 (2018); 10.1063/1.5023242

Stability of standing wave for the fractional nonlinear Schrödinger equation Journal of Mathematical Physics 59, 011508 (2018); 10.1063/1.5021689

Scattering theory for multistate Schrödinger operators Journal of Mathematical Physics 59, 012101 (2018); 10.1063/1.4989733

\section{PHYSICS TODAY}

WHITEPAPERS
MANAGER'S GUIDE

Accelerate R\&D with

Multiphysics Simulation
READ NOW

PRESENTED BY

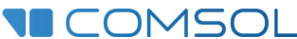




\title{
Inverse scattering transform for the nonlocal nonlinear Schrödinger equation with nonzero boundary conditions
}

\author{
Mark J. Ablowitz, ${ }^{1, a)}$ Xu-Dan Luo, ${ }^{2, b)}$ and Ziad H. Musslimani, $\left.{ }^{3, c}\right)$ \\ ${ }^{1}$ Department of Applied Mathematics, University of Colorado, Campus Box 526, Boulder, \\ Colorado 80309-0526, USA \\ ${ }^{2}$ Department of Mathematics, State University of New York at Buffalo, Buffalo, \\ New York 14260-2900, USA \\ ${ }^{3}$ Department of Mathematics, Florida State University, Tallahassee, Florida 32306-4510, USA
}

(Received 13 March 2017; accepted 4 December 2017; published online 9 January 2018)

In 2013, a new nonlocal symmetry reduction of the well-known AKNS (an integrable system of partial differential equations, introduced by and named after Mark J. Ablowitz, David J. Kaup, and Alan C. Newell et al. (1974)) scattering problem was found. It was shown to give rise to a new nonlocal $P T$ symmetric and integrable Hamiltonian nonlinear Schrödinger (NLS) equation. Subsequently, the inverse scattering transform was constructed for the case of rapidly decaying initial data and a family of spatially localized, time periodic one-soliton solutions was found. In this paper, the inverse scattering transform for the nonlocal NLS equation with nonzero boundary conditions at infinity is presented in four different cases when the data at infinity have constant amplitudes. The direct and inverse scattering problems are analyzed. Specifically, the direct problem is formulated, the analytic properties of the eigenfunctions and scattering data and their symmetries are obtained. The inverse scattering problem, which arises from a novel nonlocal system, is developed via a left-right Riemann-Hilbert problem in terms of a suitable uniformization variable and the time dependence of the scattering data is obtained. This leads to a method to linearize/solve the Cauchy problem. Pure soliton solutions are discussed, and explicit 1-soliton solution and two 2-soliton solutions are provided for three of the four different cases corresponding to two different signs of nonlinearity and two different values of the phase difference between plus and minus infinity. In another case, there are no solitons. Published by AIP Publishing. https://doi.org/10.1063/1.5018294

\section{INTRODUCTION}

Solitons are a unique type of nonlinear wave that arise as a solution to integrable infinite dimensional Hamiltonian dynamical systems. They were first discovered by Zabusky and Kruskal while conducting numerical experiments on the Korteweg-de Vries (KdV) equation. To their surprise, such solitons revealed an unusual particle-like behavior upon collisions despite the fact that they are inherently nonlinear "objects." Their results sparked intense research interest on two parallel fronts. One is related to the physics and applications of solitons (or solitary waves), while the other is focused on the mathematical structure of integrable evolution equations.

From the physics point of view, solitons, or solitary waves, represent finite energy spatially localized structures that generally form as a balance between dispersion and nonlinearity. They have been theoretically predicted and observed in laboratory experiments in various settings in the physical and optical sciences (see Refs. 16, 17, and 21 for extensive reviews).

In fluid mechanics, they have been shown to appear as isolated humps in shallow water, whereas in nonlinear optics, they occur as diffraction-free self guided nonlinear modes of a self-induced optical

\footnotetext{
a)E-mail: mark.ablowitz@ colorado.edu

b) E-mail: xudanluo@buffalo.edu

c)E-mail: muslimani@math.fsu.edu
} 
potential. Both disciplines provide exceptional situations where mathematical analysis, numerical simulations, mathematical modeling, and laboratory experiments go hand in hand.

Mathematically speaking, exactly solvable models play an essential role in the study of nonlinear wave propagation. There are many integrable equations that arise as important models in diverse physical phenomena. For example, the Korteweg-de Vries (KdV) and the Kadomtsev-Petviashvili (KP) equations describe weakly nonlinear shallow water waves ${ }^{7,18}$ propagating in one and two dimensions, respectively. The cubic nonlinear Schrödinger (NLS) equation is also a physically important integrable model. ${ }^{22}$ It describes the evolution of weakly nonlinear and quasi-monochromatic wave trains in media with cubic nonlinearities. ${ }^{8}$ Solitons appear as a special class of solutions to these models which are integrable in the sense that they admit an infinite number of conserved quantities. The KdV, KP, and NLS equations share the mathematical property that they all are exactly solvable evolution equations with many explicit solutions and linearizations known.

There are many other continuous and also discrete integrable evolution equations that are physically relevant. Applications are diverse and include problems in fluid mechanics, electromagnetics, gravitational waves, elasticity, fundamental physics, and lattice dynamics, to name but a few., 5,9

Recently, continuous and discrete integrable nonlinear nonlocal Schrödinger equations describing wave propagation in certain nonlinear PT (Parity-time) symmetric media ${ }^{20}$ were also found; remarkably, they have a very simple structure. ${ }^{1,2}$ Furthermore, reverse space-time and reverse time only nonlocal NLS equations have also been reported. ${ }^{4}$

Generally speaking, integrability is established once an infinite number of constants of motion or an infinite number of conservation laws are obtained. However, considerably more information about the solution can be obtained if the inverse scattering transform (IST) can be carried out. ${ }^{6}$

The inverse scattering transform (IST) to solve the initial-value problem with rapidly decaying data for the nonlocal NLS equation,

$$
i q_{t}(x, t)=q_{x x}(x, t)-2 \sigma q^{2}(x, t) q^{*}(-x, t),
$$

where $q^{*}(x, t)$ denotes the complex conjugate of $q(x, t), x \in \mathbb{R}, t \geq 0$ and $\sigma=\mp 1$, has been developed in Refs. 1 and 3. The direct and inverse scattering problems arise from a novel and interesting nonlocal second order system of differential equations; see system (2.1) below. Associated with this system are symmetry relations which relate analytic eigenfunctions as $x \rightarrow \infty$ to those as $x \rightarrow-\infty$. In turn, it is useful to employ Riemann-Hilbert (RH) problems from both the left and right in order to effectively develop the inverse scattering for both sets of eigenfunctions, i.e., those defined as $x \rightarrow \pm \infty$. We refer to this as left-right Riemann-Hilbert problems. This is different from the classical NLS equation where the inverse problem is carried out using a RH problem using corresponding symmetries at either infinity. ${ }^{9}$ It is important to carry out the inverse scattering analysis not only to be able to solve the nonlinear equation but also because inverse scattering is important in its own right. Equation (1.1) was derived based on physical intuition. Recently, this equation was derived in the physical context of magnetics. ${ }^{14}$

It is well-known that the IST procedure for rapidly decaying potentials must be substantially modified when one is interested in potentials that do not decay as $|x| \rightarrow \infty$. This class of potentials is also relevant for the nonlocal NLS equation since it admits soliton solutions with nonzero boundary conditions (NZBCs).

For the classical NLS equation, the first studies of NZBCs were done in Ref. 23. The method to carry out the inverse problem employed two Riemann surfaces associated with square root branch points in the eigenfunctions/scattering data. An improvement was made with the introduction of a uniformization variable. ${ }^{13}$ This transforms the inverse problem to the more standard inverse problem in the upper lower/half planes in the new variable. Subsequently, a number of researchers have also studied NLS problems in this manner, cf. Refs. 19, 11, 12, and 10.

In this paper, we consider Eq. (1.1) with the following nonzero boundary conditions:

$$
q(x, t) \rightarrow q_{ \pm}(t)=q_{0} e^{i \theta_{ \pm}(t)} \text {, as } x \rightarrow \pm \infty,
$$

where $q_{0}>0$ is a constant, $0 \leq \theta_{ \pm}<2 \pi$, and $\Delta \theta:=\theta_{+}-\theta_{-}$is either 0 or $\pi$. If $\Delta \theta:=\theta_{+}-\theta_{-} \neq$ $0, \pi$, then the amplitude $q_{ \pm}(t)$ is exponentially growing/decaying at one or the other infinity. We do not consider this situation. We consider four different cases: two different signs of $\sigma=\mp 1$ and two 
different values of $\Delta \theta=0, \pi$. First, we consider the case when $\sigma=-1, \Delta \theta=\pi$. We find the following 1 -soliton stationary in space, oscillating in time solution:

$$
q(x, t)=q_{0} \cdot e^{i\left(2 q_{0}^{2} t+\theta_{+}-\pi\right)} \cdot \tanh \left[q_{0} x-i \theta_{*}\right],
$$

where $\theta_{*}=\frac{1}{2}\left(\theta_{+}+\theta_{1}+\pi\right), \theta_{1}$ is a real constant related to the scattering data. This solution can be singular in the non-generic case when $x=0$ and $\theta_{+}+\theta_{1}=2 n \pi, n \in \mathbb{Z}$. Apart from a complex phase shift, the above solution is similar to the well known black soliton solution in the standard integrable NLS equation. Second, we consider the case when $\sigma=-1, \Delta \theta=0$. In this case, a single eigenvalue is found to be in the continuous spectrum; there is no "proper exponentially decaying" pure one-soliton solution. The simplest decaying pure reflectionless potential generates a 2-soliton standing wave solution. This is a new property of the nonlocal NLS equation (1.1) which does not appear in the classical NLS equation. Third, we consider the case when $\sigma=1, \Delta \theta=\pi$. Here all solitons must arise from an even number of eigenvalues: $2 N$. The simplest situation occurs when $N=1$ which leads to a 2-soliton traveling wave solution. Finally, the case when $\sigma=1, \Delta \theta=0$ is considered; in this case, we show that there are no eigenvalues/solitons.

We use the uniformization methodology mentioned earlier for the nonlocal NLS problem with the above NZBCs. We first introduce a two-sheeted Riemann surface and then introduce a suitable uniformization variable. There are a number of new features regarding the nonlocal NLS equation such as the introduction of important new symmetries which, when combined with a left-right RH problem allows us to construct the inverse scattering. There are situations when only an even number of solitons (eigenvalues) can be obtained and others in which there are no eigenvalues/solitons at all. In certain non-generic situations, the solitons can be singular. We also study "box"-like potentials and show that the eigenvalue spectrum is consistent with these results.

The outline of this paper is as follows. In Sec. II, some preliminaries are developed. The equation and compatible linear pair are given and the different nonzero boundary values at infinity that we will consider in this paper are presented. It is also shown that the only cases in which the amplitude at infinity are not exponentially growing/decaying are when $\sigma=\mp 1, \Delta \theta=0, \pi$. In Sec. III, the direct scattering theory is analyzed and the analytic structure of the eigenfunctions and associated scattering data are found. From the symmetry of the potentials, the corresponding symmetry of the eigenfunctions and scattering data are deduced. A suitable uniformization variable is introduced, the inverse scattering from both the right and left is developed, and pure reflectionless potentials and trace formulae are obtained. From the time dependence of the scattering data, the IST is constructed; pure soliton solutions are discussed and an explicit one-soliton solution is given.

The methodology in the other three cases follows along similar lines. However the details, due to the underlying multivalued/branching structure of the scattering data, are quite different in each case; the analysis is developed in all subsequent sections.

\section{PRELIMINARIES}

The nonlocal nonlinear Schrödinger (NLS) equation (1.1) is associated with the following $2 \times 2$ compatible system: ${ }^{1}$

$$
\begin{gathered}
v_{x}=X v=\left(\begin{array}{cc}
-i k & q(x, t) \\
\sigma q^{*}(-x, t) & i k
\end{array}\right) v, \\
v_{t}=T v=\left(\begin{array}{cc}
2 i k^{2}+i \sigma q(x, t) q^{*}(-x, t) & -2 k q(x, t)-i q_{x}(x, t) \\
-2 k \sigma q^{*}(-x, t)-i \sigma q_{x}^{*}(-x, t) & -2 i k^{2}-i \sigma q(x, t) q^{*}(-x, t)
\end{array}\right) v,
\end{gathered}
$$

where $q(x, t)$ is a complex-valued function of the real variables $x$ and $t$.

Alternatively, the space part of the compatible system may be written in the form

$$
v_{x}=(i k J+Q) v, \quad x \in \mathbb{R},
$$


where

$$
J=\left(\begin{array}{cc}
-1 & 0 \\
0 & 1
\end{array}\right), \quad Q=\left(\begin{array}{cc}
0 & q(x, t) \\
\sigma q^{*}(-x, t) & 0
\end{array}\right) .
$$

Here, $q(x, t)$ is called the potential and $k$ is a complex spectral parameter. In general, as $x \rightarrow \pm \infty, q$ $\rightarrow q_{ \pm}(t)$. Then, Eq. (1.1) simplifies to

$$
\begin{gathered}
i q_{+, t}=-2 \sigma q_{+}^{2} q_{-}^{*} \text { as } \quad x \rightarrow+\infty, \\
i q_{-, t}=-2 \sigma q_{-}^{2} q_{+}^{*} \text { as } \quad x \rightarrow-\infty .
\end{gathered}
$$

From the above equations, we find the conserved quantity

$$
q_{+} q_{-}^{*}=C_{0}, \quad C_{0} \text { is a constant. }
$$

The solutions to Eqs. (2.5) and (2.6) are then given by

$$
\begin{gathered}
q_{+}(t)=q_{0,+} e^{i \theta_{+}} e^{2 i \sigma C_{0} t} \quad \text { as } \quad x \rightarrow+\infty, \\
q_{-}(t)=q_{0,-} e^{i \theta_{-}} e^{2 i \sigma C_{0}^{*} t} \quad \text { as } \quad x \rightarrow-\infty,
\end{gathered}
$$

where $q_{0, \pm}>0$ are constant. Furthermore, since

$$
C_{0}=q_{0,+} q_{0,-} e^{i \Delta \theta}, \quad \text { where } \Delta \theta=\theta_{+}-\theta_{-}=\text {const. },
$$

then if $\Delta \theta=0$ or $\Delta \theta=\pi, C_{0}$ is real. Otherwise, it is complex and the background either grows or decays exponentially as $|t| \rightarrow \infty$. In this paper, we shall only consider the cases $\Delta \theta=0$ or $\pi$. For convenience, we will take $q_{0, \pm}=q_{0}$. There is no material difference in the analysis if we take $q_{0,+} \neq q_{0,-}$.

We also note that as $x \rightarrow \pm \infty$, the eigenfunctions of the scattering problem asymptotically satisfy

$$
\left(\begin{array}{l}
v_{1} \\
v_{2}
\end{array}\right)_{x}=\left(\begin{array}{cc}
-i k & q_{0} e^{\mp 2 \sigma q_{0}^{2} t \sin \Delta \theta} \cdot e^{i\left(2 \sigma q_{0}^{2} t \cos \Delta \theta+\theta_{ \pm}\right)} \\
\sigma q_{0} e^{ \pm 2 \sigma q_{0}^{2} t \sin \Delta \theta} \cdot e^{-i\left(2 \sigma q_{0}^{2} t \cos \Delta \theta+\theta_{\mp}\right)} & i k
\end{array}\right)\left(\begin{array}{l}
v_{1} \\
v_{2}
\end{array}\right),
$$

i.e.,

$$
v_{x}=\left(i k J+Q_{ \pm}(t)\right) v,
$$

$$
Q_{ \pm}(t)=\left(\begin{array}{cc}
0 & q_{0} e^{\mp 2 \sigma q_{0}^{2} t \sin \Delta \theta} \cdot e^{i\left(2 \sigma q_{0}^{2} t \cos \Delta \theta+\theta_{ \pm}\right)} \\
\sigma q_{0} e^{ \pm 2 \sigma q_{0}^{2} t \sin \Delta \theta} \cdot e^{-i\left(2 \sigma q_{0}^{2} t \cos \Delta \theta+\theta_{\mp}\right)} & 0
\end{array}\right),
$$

where

$$
q(x, t) \rightarrow q_{ \pm}(t)=q_{0} e^{\mp 2 \sigma q_{0}^{2} t \sin \Delta \theta} \cdot e^{i\left(2 \sigma q_{0}^{2} t \cos \Delta \theta+\theta_{ \pm}\right)} \text {, as } x \rightarrow \pm \infty .
$$

Here, $q_{0}>0,0 \leq \theta_{ \pm}<2 \pi$. We see that with the condition $\Delta \theta=0$ or $\Delta \theta=\pi$, the exponentially growing terms disappear. The exponential growth is one of the additional novel aspects exhibited by the nonlocal NLS equation (1.1).

\section{THE CASE OF $\sigma=-1$ WITH $\theta_{+}-\theta_{-}=\pi$}

\section{A. Direct scattering}

In this section, we consider the nonzero boundary conditions (NZBCs) given in (2.14) and $\sigma=-1, \Delta \theta:=\theta_{+}-\theta_{-}=\pi$. With this condition, Eq. (2.13) conveniently reduces to

$$
\frac{\partial^{2} v_{j}}{\partial x^{2}}=-\left(k^{2}+q_{0}^{2} e^{i \Delta \theta}\right) v_{j}=-\left(k^{2}-q_{0}^{2}\right) v_{j}, \quad j=1,2 .
$$

Each of the two equations has two linearly independent solutions $e^{i \lambda x}$ and $e^{-i \lambda x}$ as $|x| \rightarrow \infty$, where $\lambda=\sqrt{k^{2}-q_{0}^{2}}$. The variable $k$ is then thought of as belonging to a Riemann surface $\mathbb{K}$ consisting of 
two sheets $\mathbb{C}_{1}$ and $\mathbb{C}_{2}$ with the complex plane cut along $\left(-\infty,-q_{0}\right] \cup\left[q_{0},+\infty\right)$ with its edges glued in such a way that $\lambda(k)$ is continuous through the cut. We introduce the local polar coordinates

$$
k-q_{0}=r_{1} e^{i \theta_{1}}, \quad 0 \leq \theta_{1}<2 \pi, \quad k+q_{0}=r_{2} e^{i \theta_{2}}, \quad-\pi \leq \theta_{2}<\pi,
$$

where $r_{1}=\left|k-q_{0}\right|$ and $r_{2}=\left|k+q_{0}\right|$. Then, the function $\lambda(k)$ becomes single-valued on $\mathbb{K}$, i.e.,

$$
\lambda(k)=\left\{\begin{array}{l}
\lambda_{1}(k)=\left(r_{1} r_{2}\right)^{\frac{1}{2}} \cdot e^{i \frac{\theta_{1}+\theta_{2}}{2}}, k \in \mathbb{C}_{1}, \\
\lambda_{2}(k)=-\left(r_{1} r_{2}\right)^{\frac{1}{2}} \cdot e^{i \frac{\theta_{1}+\theta_{2}}{2}}, k \in \mathbb{C}_{2} .
\end{array}\right.
$$

Moreover, if $k \in \mathbb{C}_{1}$, then $\mathfrak{I} \lambda \geq 0$; and if $k \in \mathbb{C}_{2}$, then $\mathfrak{I} \lambda \leq 0$. Hence, the variable $\lambda$ is thought of as belonging to the complex plane consisting of the upper half plane, $U_{+}: \mathfrak{J} \lambda \geq 0$, and lower half plane, $U_{-}: \mathfrak{I} \lambda \leq 0$, glued together along the real axis; the transition occurs at $\mathfrak{I} \lambda=0$. The transformation $k \rightarrow \lambda$ maps $\mathbb{C}_{1}$ onto $U_{+}, \mathbb{C}_{2}$ onto $U_{-}$, the cut $\left(-\infty,-q_{0}\right] \cup\left[q_{0},+\infty\right)$ onto the real axis, and the points $\pm q_{0}$ to 0 ; see also Fig. 1 .

\section{B. Eigenfunctions}

It is natural to introduce the eigenfunctions defined by the following boundary conditions:

$$
\begin{gathered}
\phi(x, k) \sim w e^{-i \lambda x}, \quad \bar{\phi}(x, k) \sim \bar{w} e^{i \lambda x}, \quad \text { as } \quad x \rightarrow-\infty, \\
\psi(x, k) \sim v e^{i \lambda x}, \quad \bar{\psi}(x, k) \sim \bar{v} e^{-i \lambda x}, \quad \text { as } \quad x \rightarrow+\infty .
\end{gathered}
$$

We substitute the above conditions into (2.11), obtaining

$$
w=\left(\begin{array}{c}
\lambda+k \\
-i q_{+}^{*}
\end{array}\right), \quad \bar{w}=\left(\begin{array}{c}
-i q_{-} \\
\lambda+k
\end{array}\right), \quad v=\left(\begin{array}{c}
-i q_{+} \\
\lambda+k
\end{array}\right), \quad \bar{v}=\left(\begin{array}{c}
\lambda+k \\
-i q_{-}^{*}
\end{array}\right),
$$

which satisfy the boundary conditions, but they are not unique. In the following analysis, it is convenient to consider functions with constant boundary conditions. We define the bounded eigenfunctions as follows:

Sheet I: $\operatorname{Im} \lambda>0$

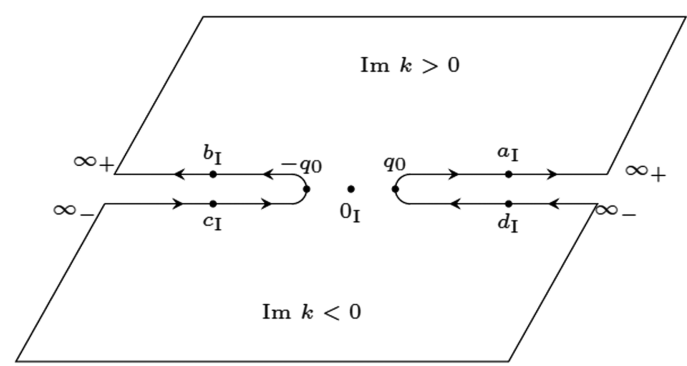

Sheet II: $\operatorname{Im} \lambda<0$

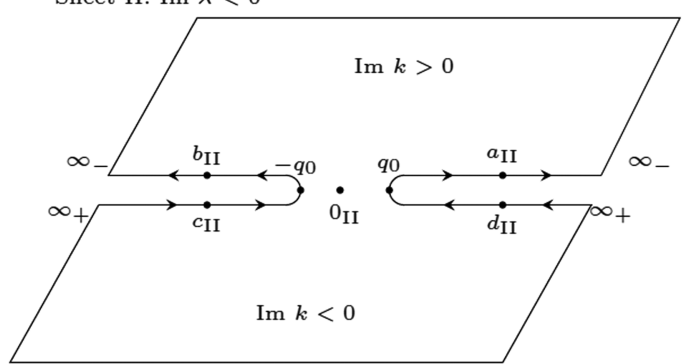

Sheet $\mathrm{I}: \operatorname{Im} \lambda>0 \quad(b)$

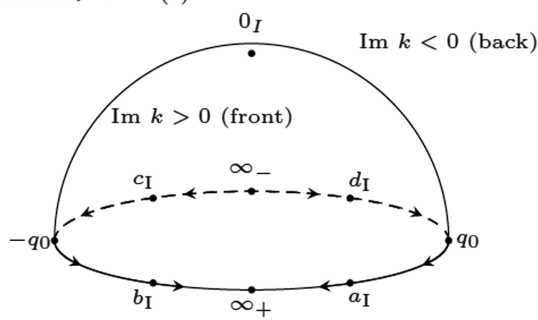

Sheet II: $\operatorname{Im} \lambda<0$

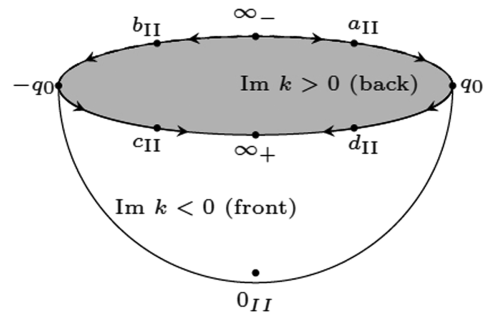

FIG. 1. (a) The two-sheeted Riemann surface $\mathbb{K}$. (b) The genus 0 surface is topologically equivalent to $\mathbb{K}$. 


$$
\begin{aligned}
& M(x, k)=e^{i \lambda x} \phi(x, k), \quad \bar{M}(x, k)=e^{-i \lambda x} \bar{\phi}(x, k), \\
& N(x, k)=e^{-i \lambda x} \psi(x, k), \quad \bar{N}(x, k)=e^{i \lambda x} \bar{\psi}(x, k) .
\end{aligned}
$$

The eigenfunctions can be represented by means of the following integral equations:

$$
\begin{aligned}
& M(x, k)=\left(\begin{array}{c}
\lambda+k \\
-i q_{+}^{*}
\end{array}\right)+\int_{-\infty}^{+\infty} G_{-}\left(x-x^{\prime}, k\right)\left(\left(Q-Q_{-}\right) M\right)\left(x^{\prime}, k\right) d x^{\prime}, \\
& \bar{M}(x, k)=\left(\begin{array}{c}
-i q_{-} \\
\lambda+k
\end{array}\right)+\int_{-\infty}^{+\infty} \bar{G}_{-}\left(x-x^{\prime}, k\right)\left(\left(Q-Q_{-}\right) \bar{M}\right)\left(x^{\prime}, k\right) d x^{\prime}, \\
& N(x, k)=\left(\begin{array}{c}
-i q_{+} \\
\lambda+k
\end{array}\right)+\int_{-\infty}^{+\infty} G_{+}\left(x-x^{\prime}, k\right)\left(\left(Q-Q_{+}\right) N\right)\left(x^{\prime}, k\right) d x^{\prime}, \\
& \bar{N}(x, k)=\left(\begin{array}{c}
\lambda+k \\
-i q_{-}^{*}
\end{array}\right)+\int_{-\infty}^{+\infty} \bar{G}_{+}\left(x-x^{\prime}, k\right)\left(\left(Q-Q_{+}\right) \bar{N}\right)\left(x^{\prime}, k\right) d x^{\prime} .
\end{aligned}
$$

Using the Fourier transform method, we get

$$
\begin{gathered}
G_{-}(x, k)=\frac{\theta(x)}{2 \lambda}\left[\left(1+e^{2 i \lambda x}\right) \lambda I-i\left(e^{2 i \lambda x}-1\right)\left(i k J+Q_{-}\right)\right], \\
\bar{G}_{-}(x, k)=\frac{\theta(x)}{2 \lambda}\left[\left(1+e^{-2 i \lambda x}\right) \lambda I+i\left(e^{-2 i \lambda x}-1\right)\left(i k J+Q_{-}\right)\right], \\
G_{+}(x, k)=-\frac{\theta(-x)}{2 \lambda}\left[\left(1+e^{-2 i \lambda x}\right) \lambda I+i\left(e^{-2 i \lambda x}-1\right)\left(i k J+Q_{+}\right)\right], \\
\bar{G}_{+}(x, k)=-\frac{\theta(-x)}{2 \lambda}\left[\left(1+e^{2 i \lambda x}\right) \lambda I-i\left(e^{2 i \lambda x}-1\right)\left(i k J+Q_{+}\right)\right],
\end{gathered}
$$

where $\theta(x)$ is the Heaviside function, i.e., $\theta(x)=1$ if $x>0$ and $\theta(x)=0$ if $x<0$.

Definition 3.1. We say $f \in L^{1}(\mathbb{R})$ if $\int_{-\infty}^{+\infty}|f(x)| d x<\infty$ and $f \in L^{1,2}(\mathbb{R})$ if $\int_{-\infty}^{+\infty}|f(x)| \cdot(1+|x|)^{2} d x$ $<\infty$.

Then we have the following result (see also Ref. 11).

Theorem 3.2. Suppose the entries of $Q-Q_{ \pm}$belong to $L^{1}(\mathbb{R})$, then for each $x \in \mathbb{R}$, the eigenfunctions $M(x, k)$ and $N(x, k)$ are continuous for $k \in \overline{\mathbb{C}}_{1} \backslash\left\{ \pm q_{0}\right\}$ and analytic for $k \in \mathbb{C}_{1}$, and $\bar{M}(x, k)$ and $\bar{N}(x, k)$ are continuous for $k \in \overline{\mathbb{C}}_{2} \backslash\left\{ \pm q_{0}\right\}$ and analytic for $k \in \mathbb{C}_{2}$. In addition, if the entries of $Q$ - $Q_{ \pm}$belong to $L^{1,2}(\mathbb{R})$, then for each $x \in \mathbb{R}$, the eigenfunctions $M(x, k)$ and $N(x, k)$ are continuous for $k \in \overline{\mathbb{C}}_{1}$ and analytic for $k \in \mathbb{C}_{1}, \bar{M}(x, k)$ and $\bar{N}(x, k)$ are continuous for $k \in \overline{\mathbb{C}}_{2}$ and analytic for $k \in \mathbb{C}_{2}$.

The proof of Theorem 3.2 employs the Neumann series; it is given in the Appendix.

Definition 3.3. The Schwartz space or space of rapidly decreasing functions on $\mathbb{R}^{n}$ is the function space

$$
S\left(\mathbb{R}^{n}\right):=\left\{f \in C^{\infty}\left(\mathbb{R}^{n}\right):\|f\|_{\alpha, \beta}<\infty, \forall \alpha, \beta \in \mathbb{Z}_{+}^{n}\right\},
$$

where $\alpha, \beta$ are multi-indices, $C^{\infty}\left(\mathbb{R}^{n}\right)$ is the set of smooth functions from $\mathbb{R}^{n}$ to $\mathbb{C}$, and $\|f\|_{\alpha, \beta}=$ $\sup _{x \in \mathbb{R}^{n}}\left|x^{\alpha} D^{\beta} f(x)\right|$. In particular, $x^{j} e^{-a|x|^{2}} \in S\left(\mathbb{R}^{n}\right)$, where $j$ is a multi-index and a is a positive real number.

Then we have the following result. 
Theorem 3.4. Suppose the entries of $Q-Q_{ \pm}$do not grow faster than $e^{-a x^{2}}$, where a is a positive real number, then for each $x \in \mathbb{R}$, the eigenfunctions $M(x, k), N(x, k), \bar{M}(x, k)$, and $\bar{N}(x, k)$ are analytic in the Riemann surface $\mathbb{K}$.

The proof of Theorem 3.4 is given in the Appendix.

\section{Scattering data}

The two eigenfunctions with fixed boundary conditions as $x \rightarrow-\infty$ are linearly independent, as are the two eigenfunctions with fixed boundary conditions as $x \rightarrow+\infty$. Indeed, if $u(x, k)=$ $\left(u_{1}(x, k), u_{2}(x, k)\right)^{T}$ and $v(x, k)=\left(v_{1}(x, k), v_{2}(x, k)\right)^{T}$ are any two solutions of (2.3), we have

$$
\frac{d}{d x} W(u, v)=0,
$$

where the Wronskian of $u$ and $v, W(u, v)$, is given by $W(u, v)=u_{1} v_{2}-u_{2} v_{1}$. From the asymptotics (3.4) and (3.5), it follows that

$$
\begin{aligned}
W(\phi, \bar{\phi}) & =\lim _{x \rightarrow-\infty} W(\phi(x, k), \bar{\phi}(x, k))=2 \lambda(\lambda+k), \\
W(\psi, \bar{\psi}) & =\lim _{x \rightarrow+\infty} W(\psi(x, k), \bar{\psi}(x, k))=-2 \lambda(\lambda+k),
\end{aligned}
$$

which proves that the functions $\phi(x, k)$ and $\bar{\phi}(x, k)$ are linearly independent, as are $\psi$ and $\bar{\psi}$, with only the branch points $\pm q_{0}$ being excluded. Hence, we can write $\phi(x, k)$ and $\bar{\phi}(x, k)$ as linear combinations of $\psi(x, k)$ and $\bar{\psi}(x, k)$, or vice versa. Thus, the relations

$$
\begin{aligned}
& \phi(x, k)=b(k) \psi(x, k)+a(k) \bar{\psi}(x, k), \\
& \bar{\phi}(x, k)=\bar{a}(k) \psi(x, k)+\bar{b}(k) \bar{\psi}(x, k)
\end{aligned}
$$

hold for any $k$ such that all four eigenfunctions exist. Combining (3.19) and (3.20), we can deduce that the scattering data satisfy the following characterization equation:

$$
a(k) \bar{a}(k)-b(k) \bar{b}(k)=1 .
$$

The scattering data can be represented in terms of Wronskians of the eigenfunctions, i.e.,

$$
\begin{gathered}
a(k)=\frac{W(\phi(x, k), \psi(x, k))}{W(\bar{\psi}(x, k), \psi(x, k))}=\frac{W(\phi(x, k), \psi(x, k))}{2 \lambda(\lambda+k)}, \\
\bar{a}(k)=-\frac{W(\bar{\phi}(x, k), \bar{\psi}(x, k))}{W(\bar{\psi}(x, k), \psi(x, k))}=-\frac{W(\bar{\phi}(x, k), \bar{\psi}(x, k))}{2 \lambda(\lambda+k)}, \\
b(k)=-\frac{W(\phi(x, k), \bar{\psi}(x, k))}{W(\bar{\psi}(x, k), \psi(x, k))}=-\frac{W(\phi(x, k), \bar{\psi}(x, k))}{2 \lambda(\lambda+k)}, \\
\bar{b}(k)=\frac{W(\bar{\phi}(x, k), \psi(x, k))}{W(\bar{\psi}(x, k), \psi(x, k))}=\frac{W(\bar{\phi}(x, k), \psi(x, k))}{2 \lambda(\lambda+k)} .
\end{gathered}
$$

Then from the analytic behavior of the eigenfunctions, we have the following theorem (see also Ref. 11).

Theorem 3.5. Suppose the entries of $Q-Q_{ \pm}$belong to $L^{1}(\mathbb{R})$, then a $(k)$ is continuous for $k \in \overline{\mathbb{C}}_{1} \backslash\left\{ \pm q_{0}\right\}$ and analytic for $k \in \mathbb{C}_{1}$, and $\bar{a}(k)$ is continuous for $k \in \overline{\mathbb{C}}_{2} \backslash\left\{ \pm q_{0}\right\}$ and analytic for $k \in \mathbb{C}_{2}$. Moreover, $b(k)$ and $\bar{b}(k)$ are continuous in $k \in\left(-\infty,-q_{0}\right) \cup\left(q_{0},+\infty\right)$. In addition, if the entries of $Q-Q_{ \pm}$belong to $L^{1,2}(\mathbb{R})$, then $a(k) \lambda(k)$ is continuous for $k \in \overline{\mathbb{C}}_{1}$ and analytic for $k \in \mathbb{C}_{1}$ and $\bar{a}(k) \lambda(k)$ is continuous for $k \in \overline{\mathbb{C}}_{2}$ and analytic for $k \in \mathbb{C}_{2}$. Moreover, $b(k) \lambda(k)$ and $\bar{b}(k) \lambda(k)$ are continuous for $k \in \mathbb{R}$. If the entries of $Q-Q_{ \pm}$do not grow faster than $e^{-a x^{2}}$, where $a$ is a positive real number, then $a(k) \lambda(k), \bar{a}(k) \lambda(k), b(k) \lambda(k)$, and $\bar{b}(k) \lambda(k)$ are analytic for $k \in \mathbb{K}$. 
Note that (3.21) and (3.22) can be written as

$$
\mu(x, k)=\rho(k) e^{2 i \lambda x} N(x, k)+\bar{N}(x, k), \quad \bar{\mu}(x, k)=N(x, k)+\bar{\rho}(k) e^{-2 i \lambda x} \bar{N}(x, k),
$$

where $\mu(x, k)=M(x, k) a^{-1}(k), \bar{\mu}(x, k)=\bar{M}(x, k) \bar{a}^{-1}(k), \rho(k)=b(k) a^{-1}(k)$, and $\bar{\rho}(k)=\bar{b}(k) \bar{a}^{-1}(k)$. Introducing the $2 \times 2$ matrices,

$$
m_{+}(x, k)=(\mu(x, k), N(x, k)), \quad m_{-}(x, k)=(\bar{N}(x, k), \bar{\mu}(x, k)),
$$

which are meromorphic in $\mathbb{C}_{1}$ and $\mathbb{C}_{2}$, respectively. Hence, we can write the Riemann-Hilbert problem or "jump" conditions in the $k$-plane as

$$
m_{+}(x, k)-m_{-}(x, k)=m_{-}(x, k)\left(\begin{array}{cc}
-\rho(k) \bar{\rho}(k) & -\bar{\rho}(k) e^{-2 i \lambda x} \\
\rho(k) e^{2 i \lambda x} & 0
\end{array}\right)
$$

on the contour $\Sigma: k \in\left(-\infty,-q_{0}\right] \cup\left[q_{0},+\infty\right)$.

Remark 3.6. For the Riemann-Hilbert problem

$$
F^{+}(\xi)-F^{-}(\xi)=F^{-}(\xi) g(\xi)
$$

on the contour $\Sigma$, where $g(\xi)$ is Hölder-continuous in $\Sigma$, we can consider the projection operators

$$
\left(P_{j}(f)\right)(k)=\frac{1}{2 \pi i} \int_{\Sigma} \frac{\lambda(k)+\lambda(\xi)}{2 \lambda(\xi)} \cdot \frac{f(\xi)}{\xi-k} d \xi, k \in \mathbb{C}_{j}, j=1,2,
$$

where $\frac{\lambda(k)+\lambda(\xi)}{2 \lambda(\xi)} \cdot \frac{d \xi}{\xi-k}$ is the Weierstrass kernel and $\int_{\Sigma}$ denotes the integral along the oriented contour in sheet I of Fig. 1. One can show that (Ref. 24)

$$
\frac{\lambda(k)+\lambda(\xi)}{2 \lambda(\xi)} \cdot \frac{d \xi}{\xi-k}=\frac{d \xi}{\xi-k}+\text { regular terms }
$$

for $(\xi, \lambda(\xi)) \rightarrow(k, \lambda(k))$. If $f_{j}(j=1,2)$ is sectionally analytic in $\mathbb{C}_{j}$ and rapidly decaying as $|k| \rightarrow \infty$ in the proper sheet, we have

$$
\begin{gathered}
\left(P_{j}\left(f_{j}\right)\right)(k)=(-1)^{j-1} f_{j}(k), \quad k \in \mathbb{C}_{j}, \\
\left(P_{j}\left(f_{l}\right)\right)(k)=0, \quad k \in \mathbb{C}_{j} .
\end{gathered}
$$

We obtain

$$
\begin{array}{ll}
F^{-}(k)=\frac{1}{2 \pi i} \int_{\Sigma} \frac{\lambda(k)+\lambda(\xi)}{2 \lambda(\xi)} \cdot \frac{F^{-}(\xi) g(\xi)}{\xi-k} d \xi, & k \in \mathbb{C}_{2}, \\
F^{+}(k)=\frac{1}{2 \pi i} \int_{\Sigma} \frac{\lambda(k)+\lambda(\xi)}{2 \lambda(\xi)} \cdot \frac{F^{-}(\xi) g(\xi)}{\xi-k} d \xi, & k \in \mathbb{C}_{1} .
\end{array}
$$

For $\xi \in \Sigma$, we can take limits from the proper sheet; they are connected by the analogue of PlemeljSokhotski formulas,

$$
\begin{aligned}
& F^{+}\left(\xi_{0}\right)=\lim _{k \rightarrow \xi_{0} \in \Sigma, k \in \mathbb{C}_{1}} \frac{1}{2 \pi i} \int_{\Sigma} \frac{\lambda(k)+\lambda(\xi)}{2 \lambda(\xi)} \cdot \frac{F^{-}(\xi) g(\xi)}{\xi-k} d \xi \\
& F^{-}\left(\xi_{0}\right)=\lim _{k \rightarrow \xi_{0} \in \Sigma, k \in \mathbb{C}_{2}} \frac{1}{2 \pi i} \int_{\Sigma} \frac{\lambda(k)+\lambda(\xi)}{2 \lambda(\xi)} \cdot \frac{F^{-}(\xi) g(\xi)}{\xi-k} d \xi
\end{aligned}
$$

\section{Symmetry reductions}

The symmetry in the potential induces a symmetry between the eigenfunctions. Indeed, if $v(x, k)=\left(v_{1}(x, k), v_{2}(x, k)\right)^{T}$ solves $(2.3)$, then $\left(v_{2}^{*}\left(-x,-k^{*}\right), v_{1}^{*}\left(-x,-k^{*}\right)\right)^{T}$ also solves (2.3). Moreover, if $k \rightarrow-k^{*}$, according to (3.3), we have $\theta_{1} \rightarrow \pi-\theta_{2}$ and $\theta_{2} \rightarrow \pi-\theta_{1}$. Hence, $\lambda_{j}^{*}\left(-k^{*}\right)=-\lambda_{j}(k)$, where $j=1,2$. Taking into account boundary conditions (3.6), we can obtain 


$$
\psi(x, k)=-\left(\begin{array}{ll}
0 & 1 \\
1 & 0
\end{array}\right) \phi^{*}\left(-x,-k^{*}\right), \quad \bar{\psi}(x, k)=-\left(\begin{array}{ll}
0 & 1 \\
1 & 0
\end{array}\right) \bar{\phi}^{*}\left(-x,-k^{*}\right) .
$$

By (3.7) and (3.8), we can get the symmetry relations of the eigenfunctions, i.e.,

$$
N(x, k)=-\left(\begin{array}{ll}
0 & 1 \\
1 & 0
\end{array}\right) M^{*}\left(-x,-k^{*}\right), \quad \bar{N}(x, k)=-\left(\begin{array}{ll}
0 & 1 \\
1 & 0
\end{array}\right) \bar{M}^{*}\left(-x,-k^{*}\right) .
$$

From the Wronskian representations for the scattering data and the above symmetry relations, we have

$$
a^{*}\left(-k^{*}\right)=a(k), \quad \bar{a}^{*}\left(-k^{*}\right)=\bar{a}(k), \quad b^{*}\left(-k^{*}\right)=-\bar{b}(k) .
$$

When using a particular single sheet for the Riemann surface of the function $\lambda^{2}=k^{2}-q_{0}^{2}$, the involution $(k, \lambda) \rightarrow(k,-\lambda)$ can only be considered across the cuts. The scattering data and eigenfunctions are defined by means of the corresponding values on the upper/lower edge of the cut; they are labeled with superscripts \pm as clarified below. Explicitly, one has

$$
\begin{array}{ll}
a^{ \pm}(k)=\frac{W\left(\phi^{ \pm}(x, k), \psi^{ \pm}(x, k)\right)}{2 \lambda^{ \pm}\left(\lambda^{ \pm}+k\right)}, & k \in\left(-\infty,-q_{0}\right] \cup\left[q_{0},+\infty\right), \\
\bar{a}^{ \pm}(k)=-\frac{W\left(\bar{\phi}^{ \pm}(x, k), \bar{\psi}^{ \pm}(x, k)\right)}{2 \lambda^{ \pm}\left(\lambda^{ \pm}+k\right)}, & k \in\left(-\infty,-q_{0}\right] \cup\left[q_{0},+\infty\right), \\
b^{ \pm}(k)=-\frac{W\left(\phi^{ \pm}(x, k), \bar{\psi}^{ \pm}(x, k)\right)}{2 \lambda^{ \pm}\left(\lambda^{ \pm}+k\right)}, & k \in\left(-\infty,-q_{0}\right] \cup\left[q_{0},+\infty\right), \\
\bar{b}^{ \pm}(k)=\frac{W\left(\bar{\phi}^{ \pm}(x, k), \psi^{ \pm}(x, k)\right)}{2 \lambda^{ \pm}\left(\lambda^{ \pm}+k\right)}, & k \in\left(-\infty,-q_{0}\right] \cup\left[q_{0},+\infty\right) .
\end{array}
$$

Using the notation $\lambda=\lambda^{+}=-\lambda^{-}$, we have the following symmetry:

$$
\phi^{\mp}(x, k)=\frac{\lambda^{\mp}+k}{-i q_{-}} \bar{\phi}^{ \pm}(x, k), \quad \psi^{\mp}(x, k)=\frac{\lambda^{\mp}+k}{-i q_{-}^{*}} \bar{\psi}^{ \pm}(x, k),
$$

for $k \in\left(-\infty,-q_{0}\right] \cup\left[q_{0},+\infty\right)$. Moreover,

$$
a^{ \pm}(k)=-\bar{a}^{\mp}(k), \quad b^{ \pm}(k)=-\frac{q_{0}^{2}}{q_{-} \cdot q_{+}} \cdot \bar{b}^{\mp}(k)
$$

for $k \in\left(-\infty,-q_{0}\right] \cup\left[q_{0},+\infty\right)$

\section{E. Uniformization coordinates}

Before discussing the properties of scattering data and solving the inverse problem, we introduce a uniformization variable $z$, defined by the conformal mapping: $z=z(k)=k+\lambda(k)$, where $\lambda=\sqrt{k^{2}-q_{0}^{2}}$, and the inverse mapping is given by $k=k(z)=\frac{1}{2}\left(z+\frac{q_{0}^{2}}{z}\right)$. Then $\lambda(z)=\frac{1}{2}\left(z-\frac{q_{0}^{2}}{z}\right)$. We observe that

(1) the upper sheet $\mathbb{C}_{1}$ and lower sheet $\mathbb{C}_{2}$ of the Riemann surface $\mathbb{K}$ are mapped onto the upper half plane $\mathbb{C}^{+}$and lower half plane $\mathbb{C}^{-}$of the complex $z$-plane, respectively;

(2) the cut $\left(-\infty,-q_{0}\right] \cup\left[q_{0},+\infty\right)$ on the Riemann surface is mapped onto the real $z$ axis;

(3) the segments $\left[-q_{0}, q_{0}\right]$ on $\mathbb{C}_{1}$ and $\mathbb{C}_{2}$ are mapped onto the upper and lower semicircles of radius $q_{0}$ and centered at the origin of the complex $z$-plane respectively.

From Theorem 3.2, the eigenfunctions $M(x, z)$ and $N(x, z)$ are analytic in the upper half $z$-plane, i.e., $z \in \mathbb{C}^{+}$, and $\bar{M}(x, z)$ and $\bar{N}(x, z)$ are analytic in the lower half $z$-plane, i.e., $z \in \mathbb{C}^{-}$. Moreover, by Theorem 3.5, we find that $a(z)$ is analytic in the upper half $z$-plane: $z \in \mathbb{C}^{+}$and $\bar{a}(z)$ is analytic in the lower half plane: $z \in \mathbb{C}^{-}$. 


\section{F. Symmetries via uniformization coordinates} Hence,

It is known that (1) when $z \rightarrow-z^{*}$, then $(k, \lambda) \rightarrow\left(-k^{*},-\lambda^{*}\right)$; (2) when $z \rightarrow \frac{q_{0}^{2}}{z}$, then $(k, \lambda) \rightarrow(k,-\lambda)$.

$$
\begin{gathered}
\psi(x, z)=-\left(\begin{array}{ll}
0 & 1 \\
1 & 0
\end{array}\right) \phi^{*}\left(-x,-z^{*}\right), \quad \bar{\psi}(x, z)=-\left(\begin{array}{ll}
0 & 1 \\
1 & 0
\end{array}\right) \bar{\phi}^{*}\left(-x,-z^{*}\right), \\
\phi\left(x, \frac{q_{0}^{2}}{z}\right)=\frac{\frac{q_{0}^{2}}{z}}{-i q_{-}} \cdot \bar{\phi}(x, z), \quad \psi\left(x, \frac{q_{0}^{2}}{z}\right)=\frac{-i q_{+}}{z} \cdot \bar{\psi}(x, z), \quad \mathfrak{J} z<0 .
\end{gathered}
$$

Similarly, we can get

$$
N(x, z)=-\left(\begin{array}{ll}
0 & 1 \\
1 & 0
\end{array}\right) M^{*}\left(-x,-z^{*}\right), \quad \bar{N}(x, z)=-\left(\begin{array}{ll}
0 & 1 \\
1 & 0
\end{array}\right) \bar{M}^{*}\left(-x,-z^{*}\right) .
$$

Moreover,

$$
\begin{gathered}
a^{*}\left(-z^{*}\right)=a(z), \quad \mathfrak{I} z>0, \quad \bar{a}^{*}\left(-z^{*}\right)=\bar{a}(z), \quad \mathfrak{I} z<0 ; \quad b^{*}\left(-z^{*}\right)=-\bar{b}(z), \\
a\left(\frac{q_{0}^{2}}{z}\right)=-\bar{a}(z), \quad \mathfrak{I} z<0 ; \quad b\left(\frac{q_{0}^{2}}{z}\right)=-\frac{q_{0}^{2}}{q_{-} \cdot q_{+}} \cdot \bar{b}(z) .
\end{gathered}
$$

We will assume that $a(z)$ and $\bar{a}(z)$ have simple zeros in the upper/lower half $z$-planes respectively. We assume that there are no multiple zeros and no zeros on $\mathfrak{J} z=0$.

\section{G. Asymptotic behavior of eigenfunctions and scattering data}

In order to solve the inverse problem, one has to determine the asymptotic behavior of both eigenfunctions and scattering data as $z \rightarrow \infty$ and as $z \rightarrow 0$. From the integral equations (in terms of Green's functions), we have

$$
\begin{gathered}
\bar{N}(x, z) \sim\left(\begin{array}{c}
z \\
-i q^{*}(-x)
\end{array}\right), \quad z \rightarrow \infty \bar{N}(x, z) \sim\left(\begin{array}{c}
z \cdot \frac{q(x)}{q_{+}} \\
-i q_{-}^{*}
\end{array}\right), \quad z \rightarrow 0, \\
a(z)=\left\{\begin{array}{ll}
1, & z \rightarrow \infty, \\
-1, & z \rightarrow 0,
\end{array} \quad \bar{a}(z)=\left\{\begin{array}{cc}
1, & z \rightarrow \infty, \\
-1, & z \rightarrow 0,
\end{array}\right.\right. \\
\lim _{z \rightarrow \infty} z b(z)=0, \quad \lim _{z \rightarrow 0} \frac{b(z)}{z^{2}}=0 .
\end{gathered}
$$

The asymptotic behavior of $M(x, z), N(x, z)$, and $\bar{M}(x, z)$ are given in the Appendix.

\section{H. Riemann-Hilbert problem via uniformization coordinates}

\section{Left scattering problem}

In order to take into account the behavior of the eigenfunctions, the "jump" conditions at the real $z$-axis can be written from the left end as

$$
\begin{aligned}
& \frac{M(x, z)}{z a(z)}-\frac{\bar{N}(x, z)}{z}=\rho(z) e^{i\left(z-\frac{q_{0}^{2}}{z}\right) x} \cdot \frac{N(x, z)}{z}, \\
& \frac{\bar{M}(x, z)}{z \bar{a}(z)}-\frac{N(x, z)}{z}=\bar{\rho}(z) e^{-i\left(z-\frac{q_{0}^{2}}{z}\right) x} \cdot \frac{\bar{N}(x, z)}{z}
\end{aligned}
$$


so that the functions will be bounded at infinity, though having an additional pole at $z=0$. Note that $M(x, z) / a(z)$, as a function of $z$, is defined in the upper half plane $\mathbb{C}^{+}$, where it has (by assumption) simple poles $z_{j}$, i.e., $a\left(z_{j}\right)=0$, and $\bar{M}(x, z) / \bar{a}(z)$ is defined in the lower half plane $\mathbb{C}^{-}$, where it has simple poles $\bar{z}_{j}$, i.e., $\bar{a}\left(\bar{z}_{j}\right)=0$. It follows that

$$
\begin{gathered}
M\left(x, z_{j}\right)=b\left(z_{j}\right) e^{i\left(z_{j}-\frac{q_{0}^{2}}{z_{j}}\right) x} \cdot N\left(x, z_{j}\right), \\
\bar{M}\left(x, \bar{z}_{j}\right)=\bar{b}\left(\bar{z}_{j}\right) e^{-i\left(\bar{z}_{j}-\frac{q_{0}^{2}}{\bar{z}_{j}}\right) x} \cdot \bar{N}\left(x, \bar{z}_{j}\right) .
\end{gathered}
$$

Then subtracting the values at infinity, the induced pole at the origin and the poles, assumed simple in the upper/lower half planes, respectively, at $a\left(z_{j}\right)=0, j=1,2, \ldots, J$ and $\bar{a}\left(\bar{z}_{j}\right), j=1,2, \ldots, \bar{J}$ (later we will see that $J=\bar{J}$ ) gives

$$
\begin{aligned}
& {\left[\frac{M(x, z)}{z a(z)}-\left(\begin{array}{l}
1 \\
0
\end{array}\right)-\frac{1}{z}\left(\begin{array}{c}
0 \\
-i q_{-}^{*}
\end{array}\right)-\sum_{j=1}^{J} \frac{M\left(x, z_{j}\right)}{\left(z-z_{j}\right) z_{j} a^{\prime}\left(z_{j}\right)}\right]} \\
& -\left[\frac{\bar{N}(x, z)}{z}-\left(\begin{array}{l}
1 \\
0
\end{array}\right)-\frac{1}{z}\left(\begin{array}{c}
0 \\
-i q_{-}^{*}
\end{array}\right)-\sum_{j=1}^{J} \frac{b\left(z_{j}\right) e^{i\left(z_{j}-\frac{q_{0}^{2}}{z_{j}}\right) x} \cdot N\left(x, z_{j}\right)}{\left(z-z_{j}\right) z_{j} a^{\prime}\left(z_{j}\right)}\right] \\
& =\rho(z) e^{i\left(z-\frac{q_{0}^{2}}{z}\right) x} \cdot \frac{N(x, z)}{z}, \\
& {\left[\frac{\bar{M}(x, z)}{z \bar{a}(z)}-\left(\begin{array}{l}
0 \\
1
\end{array}\right)-\frac{1}{z}\left(\begin{array}{c}
-i q_{+} \\
0
\end{array}\right)-\sum_{j=1}^{\bar{J}} \frac{\bar{M}\left(x, \bar{z}_{j}\right)}{\left(z-\bar{z}_{j}\right) \bar{z}_{j} a^{\prime}\left(\bar{z}_{j}\right)}\right]} \\
& -\left[\frac{N(x, z)}{z}-\left(\begin{array}{l}
0 \\
1
\end{array}\right)-\frac{1}{z}\left(\begin{array}{c}
-i q_{+} \\
0
\end{array}\right)-\sum_{j=1}^{\bar{J}} \frac{\bar{b}\left(\bar{z}_{j}\right) e^{-i\left(\bar{z}_{j}-\frac{q_{0}^{2}}{\bar{z}_{j}}\right) x} \cdot \bar{N}\left(x, \bar{z}_{j}\right)}{\left(z-\bar{z}_{j}\right) \bar{z}_{j} \bar{a}^{\prime}\left(\bar{z}_{j}\right)}\right] \\
& =\bar{\rho}(z) e^{-i\left(z-\frac{q_{0}^{2}}{z}\right) x} \cdot \frac{\bar{N}(x, z)}{z} .
\end{aligned}
$$

We now introduce the projection operators

$$
P_{ \pm}(f)(z)=\frac{1}{2 \pi i} \int_{-\infty}^{+\infty} \frac{f(\xi)}{\xi-(z \pm i 0)} d \xi
$$

which are well-defined for any function $f(\xi)$ that is integrable on the real axis. If $f_{ \pm}(\xi)$ is analytic in the upper/lower $z$-plane and $f_{ \pm}(\xi)$ is decaying at large $\xi$, then

$$
P_{ \pm}\left(f_{ \pm}\right)(z)= \pm f_{ \pm}(z), \quad P_{\mp}\left(f_{ \pm}\right)(z)=0 .
$$

Applying $P_{-}$to (3.60) and $P_{+}$to (3.61), we can obtain

$$
\begin{aligned}
& \bar{N}(x, z)=\left(\begin{array}{c}
z \\
-i q_{-}^{*}
\end{array}\right)+\sum_{j=1}^{J} \frac{z \cdot b\left(z_{j}\right) e^{i\left(z_{j}-\frac{q_{0}^{2}}{z_{j}}\right) x} \cdot N\left(x, z_{j}\right)}{\left(z-z_{j}\right) z_{j} a^{\prime}\left(z_{j}\right)}+\frac{z}{2 \pi i} \int_{-\infty}^{+\infty} \frac{\rho(\xi)}{\xi(\xi-z)} \cdot e^{i\left(\xi-\frac{q_{0}^{2}}{\xi}\right) x} \cdot N(x, \xi) d \xi \\
& N(x, z)=\left(\begin{array}{c}
-i q_{+} \\
z
\end{array}\right)+\sum_{j=1}^{\bar{J}} \frac{z \cdot \bar{b}\left(\bar{z}_{j}\right) e^{-i\left(\bar{z}_{j}-\frac{q_{0}^{2}}{\bar{z}_{j}}\right) x} \cdot \bar{N}\left(x, \bar{z}_{j}\right)}{\left(z-\bar{z}_{j}\right) \bar{z}_{j} \bar{a}^{\prime}\left(\bar{z}_{j}\right)} \frac{z}{2 \pi i} \int_{-\infty}^{+\infty} \frac{\bar{\rho}(\xi)}{\xi(\xi-z)} \cdot e^{-i\left(\xi-\frac{q_{0}^{2}}{\xi}\right) x} \cdot \bar{N}(x, \xi) d \xi
\end{aligned}
$$

Since the symmetries are between eigenfunctions defined at both $\pm \infty$, we proceed to obtain the inverse scattering integral equations defined from the right end. 


\section{Right scattering problem}

The right scattering problem can be written as

$$
\begin{aligned}
& \psi(x, z)=\alpha(z) \bar{\phi}(x, z)+\beta(z) \phi(x, z), \\
& \bar{\psi}(x, z)=\bar{\alpha}(z) \phi(x, z)+\bar{\beta}(z) \bar{\phi}(x, z),
\end{aligned}
$$

where $\alpha(z), \bar{\alpha}(z), \beta(z)$, and $\bar{\beta}(z)$ are the right scattering data. Moreover, we can get the right scattering data and left scattering data satisfy the following relations:

$$
\bar{\alpha}(z)=\bar{a}(z), \quad \alpha(z)=a(z), \quad \bar{\beta}(z)=-b(z), \quad \beta(z)=-\bar{b}(z) .
$$

Thus,

$$
\begin{aligned}
& N(x, z)=a(z) \bar{M}(x, z)-\bar{b}(z) M(x, z) e^{-i\left(z-\frac{q_{0}^{2}}{z}\right) x}, \\
& \bar{N}(x, z)=\bar{a}(z) M(x, z)-b(z) \bar{M}(x, z) e^{i\left(z-\frac{q_{0}^{2}}{z}\right) x} .
\end{aligned}
$$

The above two equations can be written as follows:

$$
\begin{aligned}
& \frac{N(x, z)}{z a(z)}-\frac{\bar{M}(x, z)}{z}=-\frac{\bar{b}(z)}{a(z)} \cdot e^{-i\left(z-\frac{q_{0}^{2}}{z}\right) x} \cdot \frac{M(x, z)}{z}, \\
& \frac{\bar{N}(x, z)}{z \bar{a}(z)}-\frac{M(x, z)}{z}=-\frac{b(z)}{\bar{a}(z)} \cdot e^{i\left(z-\frac{q_{0}^{2}}{z}\right) x} \cdot \frac{\bar{M}(x, z)}{z} .
\end{aligned}
$$

By the symmetry relations of scattering data, we have

$$
\begin{aligned}
& \frac{N(x, z)}{z a(z)}-\frac{\bar{M}(x, z)}{z}=\rho^{*}\left(-z^{*}\right) \cdot e^{-i\left(z-\frac{q_{0}^{2}}{z}\right) x} \cdot \frac{M(x, z)}{z}, \\
& \frac{\bar{N}(x, z)}{z \bar{a}(z)}-\frac{M(x, z)}{z}=\bar{\rho}^{*}\left(-z^{*}\right) \cdot e^{i\left(z-\frac{q_{0}^{2}}{z}\right) x} \cdot \frac{\bar{M}(x, z)}{z} .
\end{aligned}
$$

Using similar methods as solving the left scattering problem, we obtain

$$
\begin{gathered}
\bar{M}(x, z)=\left(\begin{array}{c}
-i q_{-} \\
z
\end{array}\right)+\sum_{j=1}^{J} \frac{-z \cdot \bar{b}\left(z_{j}\right) M\left(x, z_{j}\right) e^{-i\left(z_{j}-\frac{q_{0}^{2}}{z_{j}}\right) x}}{\left(z-z_{j}\right) z_{j} a^{\prime}\left(z_{j}\right)}+\frac{z}{2 \pi i} \int_{-\infty}^{+\infty} \frac{\rho^{*}(-\xi)}{\xi(\xi-z)} \cdot e^{-i\left(\xi-\frac{q_{0}^{2}}{\xi}\right) x} \cdot M(x, \xi) d \xi \\
M(x, z)=\left(\begin{array}{c}
z \\
-i q_{+}^{*}
\end{array}\right)+\sum_{j=1}^{\bar{J}} \frac{-z \cdot b\left(\bar{z}_{j}\right) \bar{M}\left(x, \bar{z}_{j}\right) e^{i\left(\bar{z}_{j}-\frac{q_{0}^{2}}{\bar{z}_{j}}\right) x}}{\left(z-\bar{z}_{j}\right) \bar{z}_{j} \bar{a}^{\prime}\left(\bar{z}_{j}\right)}-\frac{z}{2 \pi i} \int_{-\infty}^{+\infty} \frac{\bar{\rho}^{*}(-\xi)}{\xi(\xi-z)} \cdot e^{i\left(\xi-\frac{q_{0}^{2}}{\xi}\right) x} \cdot \bar{M}(x, \xi) d \xi
\end{gathered}
$$

\section{Recovery of the potentials}

In order to reconstruct the potential, we use asymptotics. For example, from Eq. (3.53), we have $\frac{\bar{N}_{1}(x, z)}{z} \sim \frac{q(x)}{q_{+}}$as $z \rightarrow 0$. By (3.64), we can get

$$
\frac{\bar{N}_{1}(x, z)}{z} \sim 1+\sum_{j=1}^{J} \frac{b\left(z_{j}\right) e^{i\left(z_{j}-\frac{q_{0}^{2}}{z_{j}}\right) x}}{-z_{j}^{2} a^{\prime}\left(z_{j}\right)} \cdot N_{1}\left(x, z_{j}\right)+\frac{1}{2 \pi i} \int_{-\infty}^{+\infty} \frac{\rho(\xi)}{\xi^{2}} \cdot e^{i\left(\xi-\frac{q_{0}^{2}}{\xi}\right) x} \cdot N_{1}(x, \xi) d \xi
$$

as $z \rightarrow 0$. Hence,

$$
q(x)=q_{+} \cdot\left[1+\sum_{j=1}^{J} \frac{b\left(z_{j}\right) e^{i\left(z_{j}-\frac{q_{0}^{2}}{z_{j}}\right) x}}{-z_{j}^{2} a^{\prime}\left(z_{j}\right)} \cdot N_{1}\left(x, z_{j}\right)+\frac{1}{2 \pi i} \int_{-\infty}^{+\infty} \frac{\rho(\xi)}{\xi^{2}} \cdot e^{i\left(\xi-\frac{q_{0}^{2}}{\xi}\right) x} \cdot N_{1}(x, \xi) d \xi\right] .
$$

Note that the rapidly varying phase makes the integrals well defined at $\xi=0$. 


\section{J. Closing the system}

We can find $J=\bar{J}$ from $a\left(\frac{q_{0}^{2}}{z}\right)=-\bar{a}(z)$. To close the system, by the symmetry relations between the eigenfunctions, we have

$$
\begin{aligned}
& \left(\begin{array}{c}
N_{1}(x, z) \\
N_{2}(x, z)
\end{array}\right)=\left(\begin{array}{c}
-i q_{+} \\
z
\end{array}\right)+\sum_{j=1}^{J} \frac{z \cdot \bar{b}\left(\bar{z}_{j}\right) e^{-i\left(\bar{z}_{j}-\frac{q_{0}^{2}}{\bar{z}_{j}}\right) x}}{\left(z-\bar{z}_{j}\right) \bar{z}_{j} \bar{a}^{\prime}\left(\bar{z}_{j}\right)} \cdot \\
& \left(\begin{array}{c}
\bar{z}_{j}+\sum_{l=1}^{J} \frac{\bar{z}_{j} \cdot b\left(z_{l}\right) e^{i\left(z_{l}-\frac{q_{0}^{2}}{z_{l}}\right) x}}{\left(\bar{z}_{j}-z_{l}\right) z_{l} a^{\prime}\left(z_{l}\right)} \cdot N_{1}\left(x, z_{l}\right)+\frac{\bar{z}_{j}}{2 \pi i} \int_{-\infty}^{+\infty} \frac{\rho(\xi)}{\xi\left(\xi-\bar{z}_{j}\right)} \cdot e^{i\left(\xi-\frac{q_{0}^{2}}{\xi}\right) x} \cdot N_{1}(x, \xi) d \xi \\
-i q_{-}^{*}+\sum_{l=1}^{J} \frac{\bar{z}_{j} \cdot b\left(z_{l}\right) e^{i\left(z_{l}-\frac{q_{0}^{2}}{z_{l}}\right) x}}{\left(\bar{z}_{j}-z_{l}\right) z_{l} a^{\prime}\left(z_{l}\right)} \cdot N_{2}\left(x, z_{l}\right)+\frac{\bar{z}_{j}}{2 \pi i} \int_{-\infty}^{+\infty} \frac{\rho(\xi)}{\xi\left(\xi-\bar{z}_{j}\right)} \cdot e^{i\left(\xi-\frac{q_{0}^{2}}{\xi}\right) x} \cdot N_{2}(x, \xi) d \xi
\end{array}\right) \\
& -\frac{z}{2 \pi i} \int_{-\infty}^{+\infty} \frac{\bar{\rho}(\xi)}{\xi(\xi-z)} \cdot e^{-i\left(\xi-\frac{q_{0}^{2}}{\xi}\right) x} \cdot \\
& \left(\begin{array}{c}
\xi+\sum_{l=1}^{J} \frac{\xi \cdot b\left(z_{l}\right) e^{i\left(z_{l}-\frac{q_{0}^{2}}{z_{l}}\right) x}}{\left(\xi-z_{l}\right) z_{l} a^{\prime}\left(z_{l}\right)} \cdot N_{1}\left(x, z_{l}\right)+\frac{\xi}{2 \pi i} \int_{-\infty}^{+\infty} \frac{\rho(\eta)}{\eta(\eta-\xi)} \cdot e^{i\left(\eta-\frac{q_{0}^{2}}{\eta}\right) x} \cdot N_{1}(x, \eta) d \eta \\
-i q_{-}^{*}+\sum_{l=1}^{J} \frac{\xi \cdot b\left(z_{l}\right) e^{i\left(z_{l}-\frac{q_{0}^{2}}{z_{l}}\right) x}}{\left(\xi-z_{l}\right) z_{l} a^{\prime}\left(z_{l}\right)} \cdot N_{2}\left(x, z_{l}\right)+\frac{\xi}{2 \pi i} \int_{-\infty}^{+\infty} \frac{\rho(\eta)}{\eta(\eta-\xi)} \cdot e^{i\left(\eta-\frac{q_{0}^{2}}{\eta}\right) x} \cdot N_{2}(x, \eta) d \eta
\end{array}\right) d \xi .
\end{aligned}
$$

We note that from Eq. (3.78), $q(x)$ is given in terms of the component $N_{1}$. We can use only the first component of Eq. (3.79) to find this function and hence $q(x)$. Hence to complete the inverse scattering, we reduce the problem to solving an integral equation in terms of the component $N_{1}$ only.

\section{K. Trace formula}

$a(z)$ and $\bar{a}(z)$ are analytic in the upper and lower $z$-planes, respectively. As mentioned above, we assume that $a(z)$ has simple zeros, which we call $\widetilde{z}_{i}$ and $z_{j}$, where $\mathfrak{R} \widetilde{z}_{i}=0$ and $\mathfrak{R}_{z_{j}} \neq 0$, then $-z_{j}^{*}$ is also a simple zero of $a(z)$ by $a^{*}\left(-z^{*}\right)=a(z)$. By the symmetry relation $a\left(\frac{q_{0}^{2}}{z}\right)=-\bar{a}(z)$, we can deduce that $\bar{a}(z)$ has simple zeros $\frac{q_{0}^{2}}{z_{j}},-\frac{q_{0}^{2}}{z_{j}^{*}}$, and $\frac{q_{0}^{2}}{\bar{z}_{i}}$. We define

$\gamma(z)=a(z) \cdot \prod_{j=1}^{J_{1}} \frac{z-\frac{q_{0}^{2}}{z_{j}}}{z-z_{j}} \cdot \frac{z+\frac{q_{0}^{2}}{z_{j}^{*}}}{z+z_{j}^{*}} \cdot \prod_{i=1}^{J_{2}} \frac{z-\frac{q_{0}^{2}}{\widetilde{z}_{i}}}{z-\widetilde{z}_{i}}, \quad \bar{\gamma}(z)=\bar{a}(z) \cdot \prod_{j=1}^{J_{1}} \frac{z-z_{j}}{z-\frac{q_{0}^{2}}{z_{j}}} \cdot \frac{z+z_{j}^{*}}{z+\frac{q_{0}^{2}}{z_{j}^{*}}} \cdot \prod_{i=1}^{J_{2}} \frac{z-\widetilde{z}_{i}}{z-\frac{q_{0}^{2}}{\widetilde{z}_{i}}}$,

where $2 J_{1}+J_{2}=J$. Then $\gamma(z)$ and $\bar{\gamma}(z)$ are analytic in the upper and lower $z$-planes, respectively, and have no zeros in their respective half planes. We can get

$$
\begin{gathered}
\log \gamma(z)=\frac{1}{2 \pi i} \int_{-\infty}^{+\infty} \frac{\log \gamma(\xi)}{\xi-z} d \xi, \quad \frac{1}{2 \pi i} \int_{-\infty}^{+\infty} \frac{\log \bar{\gamma}(\xi)}{\xi-z} d \xi=0, \quad \mathfrak{I} z>0, \\
\log \bar{\gamma}(z)=-\frac{1}{2 \pi i} \int_{-\infty}^{+\infty} \frac{\log \bar{\gamma}(\xi)}{\xi-z} d \xi, \quad \frac{1}{2 \pi i} \int_{-\infty}^{+\infty} \frac{\log \gamma(\xi)}{\xi-z} d \xi=0, \quad \mathfrak{I} z<0 .
\end{gathered}
$$

Adding or subtracting the above equations in each half plane, respectively, yields

$$
\log \gamma(z)=\frac{1}{2 \pi i} \int_{-\infty}^{+\infty} \frac{\log \gamma(\xi) \bar{\gamma}(\xi)}{\xi-z} d \xi, \quad \mathfrak{I} z>0, \quad \log \bar{\gamma}(z)=-\frac{1}{2 \pi i} \int_{-\infty}^{+\infty} \frac{\log \gamma(\xi) \bar{\gamma}(\xi)}{\xi-z} d \xi, \quad \mathfrak{J} z<0 .
$$


Hence,

$$
\begin{aligned}
& \log a(z)=\log \left(\prod_{j=1}^{J_{1}} \frac{z-z_{j}}{z-\frac{q_{0}^{2}}{z_{j}}} \cdot \frac{z+z_{j}^{*}}{z+\frac{q_{0}^{2}}{z_{j}^{*}}} \cdot \prod_{i=1}^{J_{2}} \frac{z-\widetilde{z}_{i}}{z-\frac{q_{0}^{2}}{\bar{z}_{i}}}\right)+\frac{1}{2 \pi i} \int_{-\infty}^{+\infty} \frac{\log \gamma(\xi) \bar{\gamma}(\xi)}{\xi-z} d \xi, \quad \mathfrak{J} z>0, \\
& \log \bar{a}(z)=\log \left(\prod_{j=1}^{J_{1}} \frac{z-\frac{q_{0}^{2}}{z_{j}}}{z-z_{j}} \cdot \frac{z+\frac{q_{0}^{2}}{z_{j}^{*}}}{z+z_{j}^{*}} \cdot \prod_{i=1}^{J_{2}} \frac{z-\frac{q_{0}^{2}}{\bar{z}_{i}}}{z-\widetilde{z}_{i}}\right)-\frac{1}{2 \pi i} \int_{-\infty}^{+\infty} \frac{\log \gamma(\xi) \bar{\gamma}(\xi)}{\xi-z} d \xi, \quad \mathfrak{J} z<0 .
\end{aligned}
$$

Note that $\gamma(z) \bar{\gamma}(z)=a(z) \bar{a}(z)$, and from the unitarity condition $a(z) \bar{a}(z)-b(z) \bar{b}(z)=1$ and the symmetry $b^{*}\left(-z^{*}\right)=-\bar{b}(z)$, we can obtain

$$
\begin{aligned}
& \log a(z)=\log \left(\prod_{j=1}^{J_{1}} \frac{z-z_{j}}{z-\frac{q_{0}^{2}}{z_{j}}} \cdot \frac{z+z_{j}^{*}}{z+\frac{q_{0}^{2}}{z_{j}^{*}}} \cdot \prod_{i=1}^{J_{2}} \frac{z-\widetilde{z}_{i}}{z-\frac{q_{0}^{2}}{\widetilde{z}_{i}}}\right)+\frac{1}{2 \pi i} \int_{-\infty}^{+\infty} \frac{\log \left(1-b(\xi) b^{*}\left(-\xi^{*}\right)\right)}{\xi-z} d \xi, \quad \mathfrak{J} z>0, \\
& \log \bar{a}(z)=\log \left(\prod_{j=1}^{J_{1}} \frac{z-\frac{q_{0}^{2}}{z_{j}}}{z-z_{j}} \cdot \frac{z+\frac{q_{0}^{2}}{z_{j}^{*}}}{z+z_{j}^{*}} \cdot \prod_{i=1}^{J_{2}} \frac{z-\frac{q_{0}^{2}}{\tilde{z}_{i}}}{z-\widetilde{z}_{i}}\right)-\frac{1}{2 \pi i} \int_{-\infty}^{+\infty} \frac{\log \left(1-b(\xi) b^{*}\left(-\xi^{*}\right)\right)}{\xi-z} d \xi, \quad \mathfrak{J} z<0 .
\end{aligned}
$$

Thus we can reconstruct $a(k), \bar{a}(k)$ in terms of the eigenvalues (zeros) and only one function $b(k)$.

\section{Discrete scattering data and their symmetries}

In order to find reflectionless potentials/solitons, we need to be able to calculate the relevant scattering data: $b\left(z_{j}\right), \bar{b}\left(\bar{z}_{j}\right), a^{\prime}\left(z_{j}\right), \bar{a}^{\prime}\left(\bar{z}_{j}\right), j=1,2, \ldots, J$. The latter functions can be calculated via the trace formulae. So we concentrate on the former. Since

$$
\begin{gathered}
N_{1}(x, z)=-M_{2}^{*}\left(-x,-z^{*}\right), \quad N_{2}(x, z)=-M_{1}^{*}\left(-x,-z^{*}\right), \\
M_{1}\left(x, z_{j}\right)=b\left(z_{j}\right) e^{i\left(z_{j}-\frac{q_{0}^{2}}{z_{j}}\right) x} \cdot N_{1}\left(x, z_{j}\right), \quad M_{2}\left(x, z_{j}\right)=b\left(z_{j}\right) e^{i\left(z_{j}-\frac{q_{0}^{2}}{z_{j}}\right) x} \cdot N_{2}\left(x, z_{j}\right),
\end{gathered}
$$

we have

$$
\begin{aligned}
& N_{1}\left(x, z_{j}\right)=-b^{*}\left(-z_{j}^{*}\right) \cdot e^{-i\left(z_{j}-\frac{q_{0}^{2}}{z_{j}}\right) x} \cdot N_{2}^{*}\left(-x,-z_{j}^{*}\right), \\
& N_{2}\left(x, z_{j}\right)=-b^{*}\left(-z_{j}^{*}\right) \cdot e^{-\left(z_{j}-\frac{q_{0}^{2}}{z_{j}}\right) x} \cdot N_{1}^{*}\left(-x,-z_{j}^{*}\right) .
\end{aligned}
$$

By rewriting (3.91), we obtain

$$
N_{2}^{*}\left(-x,-z_{j}^{*}\right)=-b\left(z_{j}\right) \cdot e^{\left(z_{j}-\frac{q_{0}^{2}}{z_{j}}\right) x} \cdot N_{1}\left(x, z_{j}\right) .
$$

Combining (3.90), we can deduce the following symmetry condition on the discrete data $b\left(z_{j}\right)$ :

$$
b\left(z_{j}\right) b^{*}\left(-z_{j}^{*}\right)=1 .
$$

Similar analysis shows that $\bar{b}\left(\bar{z}_{j}\right)$ satisfies an analogous equation $\bar{b}\left(\bar{z}_{j}\right) \bar{b}^{*}\left(-\bar{z}_{j}^{*}\right)=1$.

Also, since $a(z) \sim-1$ as $z \rightarrow 0$, from the trace formula on the real axis, we have the general symmetry constraint

$$
\prod_{j=1}^{J_{1}} \frac{\left|z_{j}\right|^{4}}{q_{0}^{4}} \cdot \prod_{i=1}^{J_{2}} \frac{\left|z_{i}\right|^{2}}{q_{0}^{2}} e^{\frac{1}{2 \pi i} \int_{-\infty}^{\infty} \log \left(1+b(\xi) b^{*}(-\xi)\right) / \xi d \xi}=1,
$$

where $2 J_{1}+J_{2}=J$. 


\section{Reflectioness potentials and soliton solutions}

Reflectioness potentials and soliton solutions, when time dependence is added, correspond to zero reflection coefficients, i.e., $\rho(\xi)=0$ and $\bar{\rho}(\xi)=0$ for all real $\xi$. We also note that from the symmetry relation $\bar{b}(z)=-b^{*}\left(-z^{*}\right)$, it follows that the reflection coefficients $\rho(z)=b(z) / a(z), \bar{\rho}(z)=\bar{b}(z) / \bar{a}(z)$ will both vanish when $b(z)=0$ for $z$ on the real axis. By substituting $z=z_{l}$ in (3.79), the system (3.79) reduces to an algebraic equation that determines the functional form of these special potentials. When time dependence is added, the reflectionless potentials correspond to soliton solutions. The reduced equations take the form

$$
\left(\begin{array}{c}
N_{1}\left(x, z_{l}\right) \\
N_{2}\left(x, z_{l}\right)
\end{array}\right)=\left(\begin{array}{c}
-i q_{+} \\
z_{l}
\end{array}\right)+\sum_{j=1}^{J} \frac{z_{l} \cdot \bar{b}\left(\bar{z}_{j}\right) e^{-i\left(\bar{z}_{j}-\frac{q_{0}^{2}}{\bar{z}_{j}}\right) x}}{\left(z_{l}-\bar{z}_{j}\right) \bar{z}_{j} \bar{a}^{\prime}\left(\bar{z}_{j}\right)} \cdot\left(\begin{array}{c}
\bar{z}_{j}+\sum_{l=1}^{J} \frac{\bar{z}_{j} \cdot b\left(z_{l} l\right) e^{i\left(z_{l}-\frac{q_{0}^{2}}{z_{l}}\right)_{x}}}{\left(\bar{z}_{j}-z_{l}\right) z_{l} a^{\prime}\left(z_{l}\right)} \cdot N_{1}\left(x, z_{l}\right) \\
-i q_{-}^{*}+\sum_{l=1}^{J} \frac{\bar{z}_{j} \cdot b\left(z_{l}\right) e^{i\left(z_{l}-\frac{q_{0}^{2}}{z_{l}}\right)_{x}}}{\left(\bar{z}_{j}-z_{l}\right) z_{l} l^{\prime}\left(z_{l}\right)} \cdot N_{2}\left(x, z_{l}\right)
\end{array}\right) .
$$

The above equation is an algebraic system to solve for $N\left(x, z_{l}\right), l=1,2, \ldots, J$. The potentials are reconstructed from Eq. (3.78) with $\rho(\xi)=0, \bar{\rho}(\xi)=0$, i.e.,

$$
q(x)=q_{+} \cdot\left[1+\sum_{j=1}^{J} \frac{b\left(z_{j}\right) e^{i\left(z_{j}-\frac{q_{0}^{2}}{z_{j}}\right) x}}{-z_{j}^{2} a^{\prime}\left(z_{j}\right)} \cdot N_{1}\left(x, z_{j}\right)\right] .
$$

As before, since $a(z) \sim-1$ as $z \rightarrow 0$, by the trace formula when $b(\xi)=0$ in the real axis, we have the following symmetry constraint for the reflectionless potentials:

$$
\prod_{j=1}^{J_{1}} \frac{\left|z_{j}\right|^{4}}{q_{0}^{4}} \cdot \prod_{i=1}^{J_{2}} \frac{\left|z_{i}\right|^{2}}{q_{0}^{2}}=1
$$

where $2 J_{1}+J_{2}=J$.

\section{N. Reflectionless potential solution: 1-Eigenvalue}

In this subsection, we show an explicit form for the 1-eigenvalue/1-soliton solution without time dependence, by setting $J=1$. Then $J_{1}=0$ and $J_{2}=1$. Now let $\widetilde{z}_{1}=i v_{1}$, we have $\overline{\bar{z}}_{1}=-i \frac{q_{0}^{2}}{v_{1}}:=-i \bar{v}_{1}$, where $v_{1}$ is real and positive. Hence, we have

$$
N_{1}\left(x, i v_{1}\right)=\frac{-i q_{+}+\frac{v_{1} \bar{b}\left(-i \bar{v}_{1}\right) e^{-\left(\bar{v}_{1}+\frac{q_{0}^{2}}{\bar{v}_{1}}\right)_{x}}}{\left(v_{1}+\bar{v}_{1}\right) \bar{a}^{\prime}\left(-i \bar{v}_{1}\right)}}{1-\frac{\bar{b}\left(-i \bar{v}_{1}\right) b\left(i v_{1}\right) e^{-\left(v_{1}+\bar{v}_{1}+\frac{q_{0}^{2}}{v_{1}}+\frac{q_{0}^{2}}{\bar{v}_{1}}\right) x}}{\left(v_{1}+\bar{v}_{1}\right)^{2} \bar{a}^{\prime}\left(-i \bar{v}_{1}\right) a^{\prime}\left(i v_{1}\right)}} .
$$

Therefore,

$$
q(x)=q_{+} \cdot\left[1+\frac{b\left(i v_{1}\right) e^{-\left(v_{1}+\frac{q_{0}^{2}}{v_{1}}\right) x}}{v_{1}^{2} \cdot a^{\prime}\left(i v_{1}\right)} \cdot N_{1}\left(x, i v_{1}\right)\right] .
$$

By the trace formula when $b(\xi)=0$ in the real axis, we can get

$$
a^{\prime}\left(i v_{1}\right)=\frac{1}{i\left(v_{1}+\bar{v}_{1}\right)}, \quad \bar{a}^{\prime}\left(-i \bar{v}_{1}\right)=\frac{i}{v_{1}+\bar{v}_{1}} .
$$

From (3.97), we can deduce $v_{1}=\bar{v}_{1}=q_{0}$. Hence,

$$
a^{\prime}\left(i v_{1}\right)=a^{\prime}\left(i q_{0}\right)=\frac{1}{2 i q_{0}}, \quad \bar{a}^{\prime}\left(-i \bar{v}_{1}\right)=\bar{a}^{\prime}\left(-i q_{0}\right)=\frac{i}{2 q_{0}} .
$$

By choosing $\widetilde{z}_{1}=i q_{0}$ in (3.93), we can get $\left|b\left(i q_{0}\right)\right|^{2}=1$. Similarly, we have $\left|\bar{b}\left(-i q_{0}\right)\right|^{2}=1$. Thus, we write $b\left(i q_{0}\right)=e^{i \theta_{1}}$ and $\bar{b}\left(-i q_{0}\right)=e^{i \bar{\theta}_{1}}$, where both $\theta_{1}$ and $\bar{\theta}_{1}$ are real. Moreover, we can obtain $\bar{b}\left(i q_{0}\right)=-e^{-i \theta_{1}}$ and $b\left(-i q_{0}\right)=-e^{-i \bar{\theta}_{1}}$. Moreover, from (3.52), we can also get 


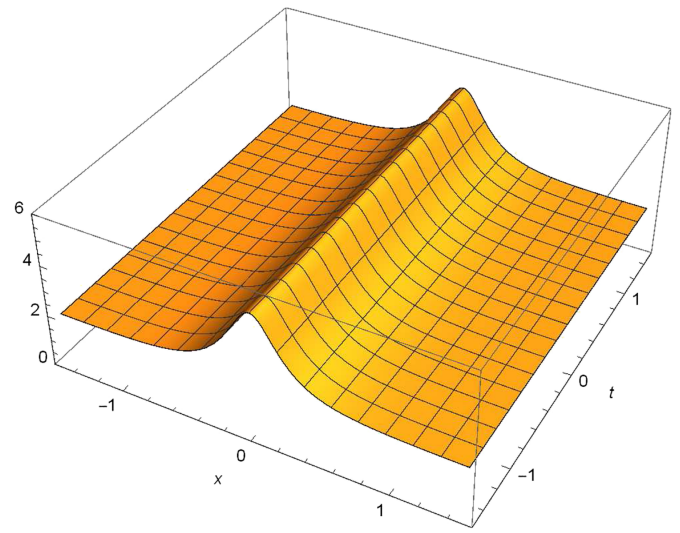

(a)

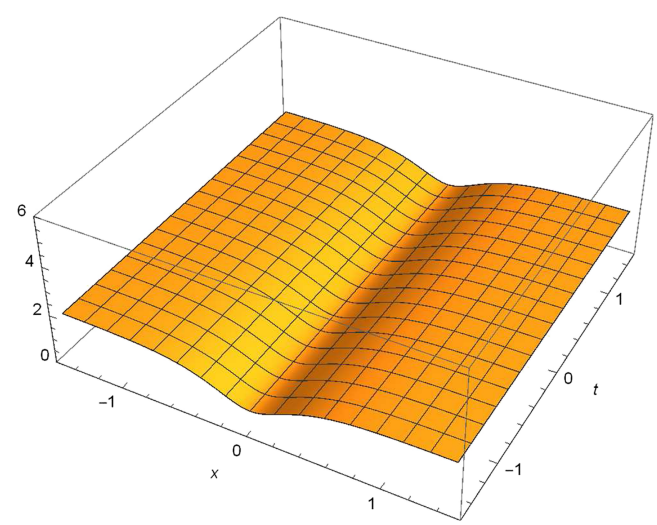

(b)

FIG. 2. (a) The amplitude of $q(x, t)$ with $\theta_{+}=\frac{\pi}{4}, \theta_{1}=0$, and $q_{0}=2$. (b) The amplitude of $q(x, t)$ with $\theta_{+}=\frac{5 \pi}{4}, \theta_{1}=0$, and $q_{0}=2$.

$$
e^{i \theta_{1}}=b\left(i q_{0}\right)=b\left(\frac{q_{0}^{2}}{-i q_{0}}\right)=-\frac{\bar{b}\left(-i q_{0}\right)}{e^{i\left(\theta_{+}+\theta_{-}\right)}}=\frac{e^{i \bar{\theta}_{1}}}{e^{2 i \theta_{+}}},
$$

i.e., $\bar{\theta}_{1}=\theta_{1}+2 \theta_{+}+2 n \pi$, where $n \in \mathbb{Z}$. Thus, we have the reflectionless potential corresponding to one eigenvalue,

$$
q(x)=q_{0} e^{i \theta_{+}} \cdot\left[1+\frac{2 e^{i \theta_{1}-2 q_{0} x} \cdot\left(e^{i \theta_{+}}+e^{i\left(\theta_{1}+2 \theta_{+}\right)-2 q_{0} x}\right)}{1-e^{2 i\left(\theta_{1}+\theta_{+}\right)-4 q_{0} x}}\right] .
$$

To find the corresponding soliton solution, we need the time evolution of the data which we derive next.

\section{O. Time evolution}

We deduce that both $a(t)$ and $\bar{a}(t)$ are time independent, and

$$
\begin{gathered}
b\left(i q_{0}, t\right)=b\left(i q_{0}, 0\right) e^{-2 i\left(q_{0}^{2}+2 \lambda k\right) t}=e^{i \theta_{1}} \cdot e^{-2 i q_{0}^{2} t}, \\
\bar{b}\left(-i q_{0}, t\right)=\bar{b}\left(-i q_{0}, 0\right) e^{2 i\left(q_{0}^{2}+2 \lambda k\right) t}=e^{i \bar{\theta}_{1}} \cdot e^{2 i q_{0}^{2} t}=e^{i\left(\theta_{1}+2 \theta_{+}\right)} \cdot e^{2 i q_{0}^{2} t} .
\end{gathered}
$$

The details are shown in the Appendix. Putting all the above into the formula we had for the reflectionless potential (3.103), we obtain the following one-soliton solution:

$$
q(x, t)=q_{0} \cdot e^{i\left(2 q_{0}^{2} t+\theta_{+}-\pi\right)} \cdot \tanh \left[q_{0} x-i \theta_{*}\right],
$$

where $\theta_{*}=\frac{1}{2}\left(\theta_{+}+\theta_{1}+\pi\right)$. This is similar to the well-known black soliton of the standard integrable NLS equation with only a complex phase shift difference. From this solution, we see that there is a singularity only when $\theta_{+}+\theta_{1}=0, \pm 2 \pi$ which puts a restriction on the otherwise arbitrary phases $\theta_{+}, \theta_{1}$. The magnitude of the solution is stationary. In Fig. 2 we give typical one-soliton solutions. We see in Fig. 2(a) that the magnitude rises from a constant background, whereas in Fig. 2(b), the magnitude dips from the constant background.

\section{THE CASE OF $\sigma=-1$ WITH $\theta_{+}-\theta_{-}=0$}

In this section, we consider the nonzero boundary conditions (NZBCs) given in (2.14) with $\sigma=$ $-1, \theta_{+}=\theta_{-}:=\theta$,

$$
q(x, t) \rightarrow q_{ \pm}(t):=\widetilde{q}(t)=q_{0} e^{-2 i q_{0}^{2} t+i \theta} \text {, as } x \rightarrow \pm \infty,
$$

where $q_{0}>0,0 \leq \theta<2 \pi$ 


\section{A. Direct scattering}

With this condition, Eq. (2.13) conveniently reduces to

$$
\frac{\partial^{2} v_{j}}{\partial x^{2}}=-\left(k^{2}+q_{0}^{2}\right) v_{j}, \quad j=1,2
$$

Each of the two equations has two linearly independent solutions $e^{i \lambda x}$ and $e^{-i \lambda x}$ as $|x| \rightarrow \infty$, where $\lambda=\sqrt{k^{2}+q_{0}^{2}}$. We introduce the local polar coordinates

$$
k-i q_{0}=r_{1} e^{i \theta_{1}}, \quad-\frac{\pi}{2} \leq \theta_{1}<\frac{3 \pi}{2}, \quad k+i q_{0}=r_{2} e^{i \theta_{2}}, \quad-\frac{\pi}{2} \leq \theta_{2}<\frac{3 \pi}{2},
$$

where $r_{1}=\left|k-i q_{0}\right|$ and $r_{2}=\left|k+i q_{0}\right|$. One can write $\lambda(k)=\left(r_{1} r_{2}\right)^{\frac{1}{2}} \cdot e^{i \cdot \frac{\theta_{1}+\theta_{2}}{2}+i m \pi}, m=0,1$, respectively, on sheets I $\left(\mathbb{K}_{1}\right)$ and II $\left(\mathbb{K}_{2}\right)$. The variable $k$ is then thought of as belonging to a Riemann surface $\mathbb{K}$ consisting of sheets I and II with both coinciding with the complex plane cut along $\Sigma:=\left[-i q_{0}\right.$, $i q_{0}$ ] with its edges glued in such a way that $\lambda(k)$ is continuous through the cut. Along the real $k$ axis, we have $\lambda(k)= \pm \operatorname{sign}(k) \sqrt{k^{2}+q_{0}^{2}}$, where the plus/minus signs apply, respectively, on sheet I and sheet II of the Riemann surface, and where the square root sign denotes the principal branch of the real-valued square root function. We denote $\mathbb{C}^{ \pm}$as the open upper/lower complex half planes, and we denote $\mathbb{K}^{ \pm}$as the open upper/lower complex half planes cut along $\Sigma$. Then $\lambda$ provides one-to-one correspondences between the following sets:

1. $k \in \mathbb{K}^{+}=\mathbb{C}^{+} \backslash\left(0, i q_{0}\right]$ and $\lambda \in \mathbb{C}^{+}$,

2. $k \in \partial \mathbb{K}^{+}=\mathbb{R} \cup\left\{i s-0^{+}: 0<s<q_{0}\right\} \cup\left\{i q_{0}\right\} \cup\left\{i s+0^{+}: 0<s<q_{0}\right\}$ and $\lambda \in \mathbb{R}$,

3. $k \in \mathbb{K}^{-}=\mathbb{C}^{-} \backslash\left[-i q_{0}, 0\right)$ and $\lambda \in \mathbb{C}^{-}$,

4. $k \in \partial \mathbb{K}^{-}=\mathbb{R} \cup\left\{i s-0^{+}:-q_{0}<s<0\right\} \cup\left\{-i q_{0}\right\} \cup\left\{i s+0^{+}:-q_{0}<s<0\right\}$ and $\lambda \in \mathbb{R}$.

The two sheets of the Riemann surface $\mathbb{K}$ are shown in Fig. 3.

Moreover, $\lambda^{ \pm}(k)$ will denote the boundary values taken by $\lambda(k)$ for $k \in \Sigma$ from the right/left edge of the cut, with $\lambda^{ \pm}(k)= \pm \sqrt{q_{0}^{2}-|k|^{2}}, k=i s \pm 0^{+},|s|<q_{0}$ on the right/left edge of the cut.

See also Fig. 3. The contours along the real axis encircling $\pm i q_{0}$ are used in a Riemann-Hilbert formulation.

Remark 4.1. Topologically, the Riemann surface $\mathbb{K}$ is equivalent to a surface with genus 0 .

Sheet I: (a)

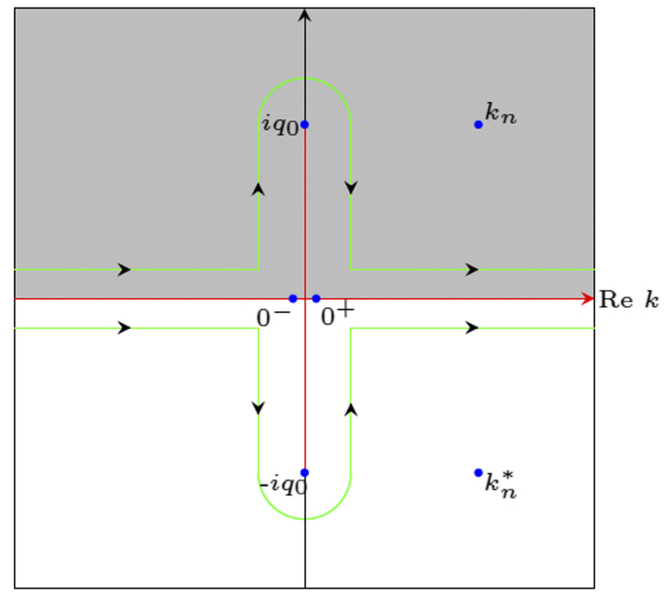

Sheet II: (b) $\quad \operatorname{Im} k$

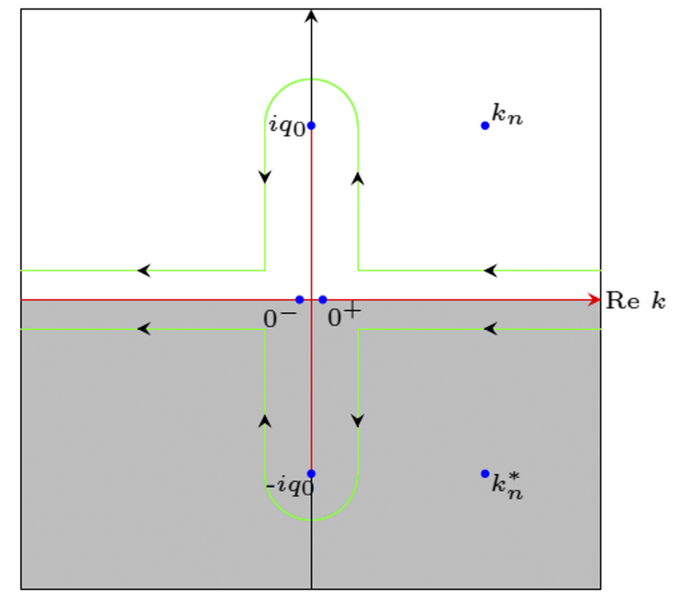

FIG. 3. (a) The first sheet of the Riemann surface, showing the branch cut (red), the contour (green), and the regions when $\mathfrak{J} \lambda>0$ (gray) and $\mathfrak{J} \lambda<0$ (white), where $\lambda(k)=\left(r_{1} r_{2}\right)^{\frac{1}{2}} e^{i \frac{\theta_{1}+\theta_{2}}{2}}$. (b) The second sheet of the Riemann surface, showing the branch cut (red), the contour (green), and the regions when $\mathfrak{J} \lambda<0$ (gray) and $\mathfrak{J} \lambda>0$ (white), where $\lambda(k)=-\left(r_{1} r_{2}\right)^{\frac{1}{2}} e^{i \frac{\theta_{1}+\theta_{2}}{2}}$. 
The eigenfunctions are defined by the following boundary conditions:

$$
\begin{gathered}
\phi(x, k) \sim w e^{-i \lambda x}, \quad \bar{\phi}(x, k) \sim \bar{w} e^{i \lambda x} \text { as } x \rightarrow-\infty, \\
\psi(x, k) \sim v e^{i \lambda x}, \quad \bar{\psi}(x, k) \sim \bar{v} e^{-i \lambda x} \text { as } \quad x \rightarrow+\infty,
\end{gathered}
$$

where

$$
w=\left(\begin{array}{c}
\lambda+k \\
-\tilde{q}^{*}
\end{array}\right), \quad \bar{w}=\left(\begin{array}{c}
-\tilde{q} \widetilde{q} \\
\lambda+k
\end{array}\right), \quad v=\left(\begin{array}{c}
-\tilde{q} \widetilde{q} \\
\lambda+k
\end{array}\right), \quad \bar{v}=\left(\begin{array}{c}
\lambda+k \\
-i \widetilde{q}^{*}
\end{array}\right)
$$

satisfy the boundary conditions, but they are not unique.

As in Sec. III, we consider functions with constant boundary conditions and define the same bounded eigenfunctions $M(x, k), N(x, k), \bar{M}(x, k), \bar{N}(x, k)$ except we use the boundary conditions defined in (4.6).

The bounded eigenfunctions can be represented by means of integral equations, the formulae are the same as (3.9)-(3.12) except for the constant terms, which are defined in (4.6).

Definition 4.2. We say $f \in L^{1,1}(\mathbb{R})$ if $\int_{-\infty}^{+\infty}|f(x)| \cdot(1+|x|) d x<\infty$.

Then, using similar methods as in the prior case $(\sigma=-1, \Delta \theta=\pi)$, we find the following result (see also Ref. 12)

Theorem 4.3. Suppose the entries of $Q-Q_{ \pm}$belong to $L^{1,1}(\mathbb{R})$, then for each $x \in \mathbb{R}$, the eigenfunctions $M(x, k)$ and $N(x, k)$ are continuous for $k \in \overline{\mathbb{K}^{+}} \cup \partial \overline{\mathbb{K}^{-}}$and analytic for $k \in \mathbb{K}^{+}$, and $\bar{M}(x, k)$ and $\bar{N}(x, k)$ are continuous for $k \in \overline{\mathbb{K}^{-}} \cup \partial \overline{\mathbb{K}^{+}}$and analytic for $k \in \mathbb{K}^{-}$.

If we assume that the entries of $Q-Q_{ \pm}$do not grow faster than $e^{-a x^{2}}$, where $a$ is a positive real number, by similar methods as in case 1 , we have the following result.

Theorem 4.4. Suppose the entries of $Q-Q_{ \pm}$do not grow faster than $e^{-a x^{2}}$, where a is a positive real number, then for each $x \in \mathbb{R}$, the eigenfunctions $M(x, k), N(x, k), \bar{M}(x, k)$, and $\bar{N}(x, k)$ are analytic in the Riemann surface $\mathbb{K}$.

\section{Scattering data}

As in Sec. III C, we can define the scattering data $a(k), \bar{a}(k), b(k)$, and $\bar{b}(k)$ in the same way and find the same representations in terms of eigenfunctions. When $k \in\left(-i q_{0}, i q_{0}\right)$, the scattering data and eigenfunctions are defined by means of the corresponding values on the right/left edge of the cut, are labeled with superscripts \pm as clarified below. Explicitly, one has the same formulae as in (3.42)-(3.45). Then from the analytic behavior of the eigenfunctions, we have the following theorem.

Theorem 4.5. Suppose the entries of $Q-Q_{ \pm}$belong to $L^{1,1}(\mathbb{R})$, then a $(k)$ is continuous for $k \in \overline{\mathbb{K}^{+}} \cup \partial \overline{\mathbb{K}^{-}} \backslash\left\{ \pm i q_{0}\right\}$ and analytic for $k \in \mathbb{K}^{+}$, and $\bar{a}(k)$ is continuous for $k \in \overline{\mathbb{K}^{-}} \cup \partial \overline{\mathbb{K}^{+}} \backslash\left\{ \pm i q_{0}\right\}$ and analytic for $k \in \mathbb{K}^{-}$. Moreover, $b(k)$ and $\bar{b}(k)$ are continuous in $k \in \mathbb{R} \cup\left(-i q_{0}, i q_{0}\right)$. In addition, if the entries of $Q-Q_{ \pm}$do not grow faster than $e^{-a x^{2}}$, where a is a positive real number, then $a(k) \lambda(k)$, $\bar{a}(k) \lambda(k), b(k) \lambda(k)$, and $\bar{b}(k) \lambda(k)$ are analytic for $k \in \mathbb{K}$.

\section{B. Symmetry reductions}

The symmetry in the potential induces a symmetry between the eigenfunctions. We can obtain the same symmetry relations as in (3.39) because we consider the same sign of $\sigma$ in Sec. III and IV. Moreover, the symmetry between scattering data is also the same as in (3.41).

When using a particular single sheet for the Riemann surface of the function $\lambda^{2}=k^{2}+q_{0}^{2}$, the involution $(k, \lambda) \rightarrow(k,-\lambda)$ leads to relationships between eigenfunctions across the cut; namely, it relates the values of eigenfunctions and scattering data for the same value of $k$ from either side of the cut. One has

$$
\phi^{\mp}(x, k)=\frac{-\lambda^{ \pm}+k}{-\widetilde{q}} \cdot \bar{\phi}^{ \pm}(x, k), \quad \psi^{\mp}(x, k)=\frac{-\lambda^{ \pm}+k}{-i \widetilde{q}^{*}} \cdot \bar{\psi}^{ \pm}(x, k)
$$


for $k \in\left[-i q_{0}, i q_{0}\right]$. Similarly,

$$
M^{\mp}(x, k)=\frac{-\lambda^{ \pm}+k}{-i \widetilde{q}} \cdot \bar{M}^{ \pm}(x, k), \quad N^{\mp}(x, k)=\frac{-\lambda^{ \pm}+k}{-i \widetilde{q}^{*}} \cdot \bar{N}^{ \pm}(x, k),
$$

for $k \in\left[-i q_{0}, i q_{0}\right]$. Then,

$$
a^{ \pm}(k)=\bar{a}^{\mp}(k), \quad b^{ \pm}(k)=\frac{\widetilde{q}^{*}}{\widetilde{q}} \cdot \bar{b}^{\mp}(k)
$$

for $k \in\left[-i q_{0}, i q_{0}\right]$.

\section{Riemann-Hilbert problem}

We will develop the Riemann-Hilbert problem across $\partial \mathbb{K}^{+} \cup \partial \mathbb{K}^{-}$.

(1) Riemann-Hilbert problem across the real axis [see Fig. 4(a)]. Note that (3.21) and (3.22) can be written as

$$
\mu(x, k)=\rho(k) e^{2 i \lambda x} N(x, k)+\bar{N}(x, k), \quad \bar{\mu}(x, k)=N(x, k)+\bar{\rho}(k) e^{-2 i \lambda x} \bar{N}(x, k),
$$

where $\mu(x, k)=M(x, k) a^{-1}(k), \bar{\mu}(x, k)=\bar{M}(x, k) \bar{a}^{-1}(k), \rho(k)=b(k) a^{-1}(k)$, and $\bar{\rho}(k)=\bar{b}(k) \bar{a}^{-1}(k)$. Introducing the $2 \times 2$ matrices,

$$
m_{+}(x, k)=(\mu(x, k), N(x, k)), \quad m_{-}(x, k)=(\bar{N}(x, k), \bar{\mu}(x, k)) .
$$

Hence, we can write the "jump" conditions as

$$
m_{+}(x, k)-m_{-}(x, k)=m_{-}(x, k)\left(\begin{array}{cc}
-\rho(k) \bar{\rho}(k) & -\bar{\rho}(k) e^{-2 i \lambda x} \\
\rho(k) e^{2 i \lambda x} & 0
\end{array}\right)
$$

for $k \in \mathbb{R}$.

(2) Riemann-Hilbert problem across [0, iq $q_{0}$ [see Fig. 4(b)]. By (3.21) and (3.22), the notation $\lambda$ $=\lambda^{+}=-\lambda^{-}$and the symmetry relations of Jost functions and scattering data, we have

$$
\frac{M^{+}(x, k)}{a^{+}(k)}=\frac{b^{+}(k)}{a^{+}(k)} \cdot N^{+}(x, k) \cdot e^{2 i \lambda x}+\frac{-i \widetilde{q}^{*}}{\lambda^{-}+k} \cdot N^{-}(x, k),
$$
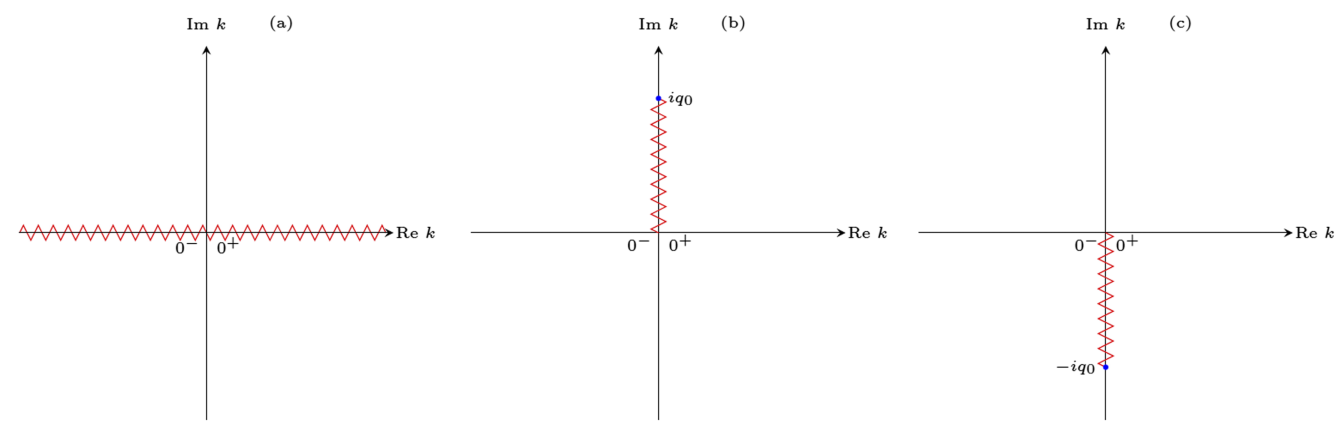

FIG. 4. (a) Riemann-Hilbert problem across the real axis. (b) Riemann-Hilbert problem across $\left[0, i q_{0}\right]$. (c) Riemann-Hilbert problem across $\left[-i q_{0}, 0\right)$. 


$$
\frac{-i q_{-}}{\lambda^{-}+k} \cdot \frac{M^{-}(x, k)}{a^{-}(k)}=N^{+}(x, k)+\frac{\widetilde{q}}{\widetilde{q}^{*}} \cdot \frac{b^{-}(k)}{a^{-}(k)} \cdot \frac{-i \widetilde{q}^{*}}{\lambda^{-}+k} \cdot N^{-}(x, k) \cdot e^{-2 i \lambda x} .
$$

Introducing the $2 \times 2$ matrices

$$
m^{+}(x, k)=\left(\mu^{+}(x, k), N^{+}(x, k)\right), \quad m^{-}(x, k)=\left(\frac{-i \widetilde{q}^{*}}{\lambda^{-}+k} N^{-}(x, k), \frac{-i \widetilde{q}}{\lambda^{-}+k} \cdot \mu^{-}(x, k)\right),
$$

where $\mu^{+}(x, k)=M^{+}(x, k) a^{+}(k)^{-1}, \mu^{-}(x, k)=M^{-}(x, k) a^{-}(k)^{-1}, \rho^{+}(k)=b^{+}(k) a^{+}(k)^{-1}$, and $\rho^{-}(k)=$ $b^{-}(k) a^{-}(k)^{-1}$. Then,

$$
m^{+}(x, k)-m^{-}(x, k)=m^{-}(x, k)\left(\begin{array}{cc}
-\frac{\widetilde{q}}{\widetilde{q}^{*}} \cdot \rho^{+}(k) \rho^{-}(k) & -\frac{\widetilde{q}}{\widetilde{q}^{*}} \cdot \rho^{-}(k) e^{-2 i \lambda x} \\
\rho^{+}(k) e^{2 i \lambda x} & 0
\end{array}\right)
$$

for $k \in \mathbb{C}^{+}$

(3) Riemann-Hilbert problem across [-iq 0 , 0) [see Fig. 4(c)]. By (3.21) and (3.22), the notation $\lambda$ $=\lambda^{+}=-\lambda^{-}$, and the symmetry relations of Jost functions and scattering data, we can get

$$
\begin{gathered}
\frac{-\lambda^{-}+k}{-i \widetilde{q}} \cdot \frac{\bar{M}^{-}(x, k)}{\bar{a}^{-}(x, k)}=\frac{\widetilde{q}^{*}}{\widetilde{q}} \cdot \frac{\bar{b}^{-}(k)}{\bar{a}^{-}(k)} \cdot \frac{-\lambda^{-}+k}{-i \widetilde{q}^{*}} \cdot \bar{N}^{-}(x, k) \cdot e^{2 i \lambda x}+\bar{N}^{+}(x, k), \\
\frac{\bar{M}^{+}(x, k)}{\bar{a}^{+}(k)}=\frac{-\lambda^{-}+k}{-i \widetilde{q}^{*}} \cdot \bar{N}^{-}(x, k)+\frac{\bar{b}^{+}(k)}{\bar{a}^{+}(k)} \cdot \bar{N}^{+}(x, k) \cdot e^{-2 i \lambda x} .
\end{gathered}
$$

Introducing the $2 \times 2$ matrices,

$$
\bar{m}^{+}(x, k)=\left(\bar{\mu}^{+}(x, k), \bar{N}^{+}(x, k)\right), \quad \bar{m}^{-}(x, k)=\left(\frac{-\lambda^{-}+k}{-i \widetilde{q}^{*}} \cdot \bar{N}^{-}(x, k), \frac{-\lambda^{-}+k}{-i \widetilde{q}} \cdot \bar{\mu}^{-}(k)\right),
$$

where $\bar{\mu}^{+}(x, k)=\bar{M}^{+}(x, k) \bar{a}^{+}(k)^{-1}, \bar{\mu}^{-}(x, k)=\bar{M}^{-}(x, k) \bar{a}^{-}(k)^{-1}, \bar{\rho}^{+}(k)=\bar{b}^{+}(k) \bar{a}^{+}(k)^{-1}$, and $\bar{\rho}^{-}(k)=$ $\bar{b}^{-}(k) \bar{a}^{-}(k)^{-1}$. Then,

$$
\bar{m}^{+}(x, k)-\bar{m}^{-}(x, k)=\bar{m}^{-}(x, k)\left(\begin{array}{cc}
-\frac{\widetilde{q}^{*}}{\widetilde{q}} \cdot \bar{\rho}^{+}(k) \bar{\rho}^{-}(k) & -\frac{\widetilde{q}^{*}}{\widetilde{q}} \cdot \bar{\rho}^{-}(k) \cdot e^{2 i \lambda x} \\
\bar{\rho}^{+}(k) e^{-2 i \lambda x} & 0
\end{array}\right)
$$

for $k \in \mathbb{C}^{-}$.

Remark 4.6. For the Riemann-Hilbert problem

$$
F^{+}(\xi)-F^{-}(\xi)=F^{-}(\xi) g(\xi)
$$

on the contour $\widetilde{\Sigma}:=\Sigma_{1} \cup \Sigma_{2} \cup \Sigma_{3}$, where $\Sigma_{1}:=\mathbb{R}, \Sigma_{2}:=\left[0, i q_{0}\right], \Sigma_{3}:=\left[-i q_{0}, 0\right), g(\xi)$ is Höldercontinuous in $\widetilde{\Sigma}$, and $g(\xi)=g_{m}(\xi)$ is chosen depending on which piece of the contour is considered, where $m=1,2,3$. We can consider the projection operators

$$
\left(P_{j}(f)\right)(k)=\frac{1}{2 \pi i} \int_{\widetilde{\Sigma}} \frac{\lambda(k)+\lambda(\xi)}{2 \lambda(\xi)} \cdot \frac{f(\xi)}{\xi-k} d \xi, \quad k \in \mathbb{C}_{j}, j=1,2,
$$

where $\frac{\lambda(k)+\lambda(\xi)}{2 \lambda(\xi)} \cdot \frac{d \xi}{\xi-k}$ is the Weierstrass kernel, $\int_{\widetilde{\Sigma}}:=\int_{\Sigma_{1} \cup \Sigma_{2}}+\int_{\Sigma_{1} \cup \Sigma_{3}}$ denote the integrals along the oriented contours in Fig. 3(a). One can show that (Ref. 24)

$$
\frac{\lambda(k)+\lambda(\xi)}{2 \lambda(\xi)} \cdot \frac{d \xi}{\xi-k}=\frac{d \xi}{\xi-k}+\text { regular terms }
$$

for $(\xi, \lambda(\xi)) \rightarrow(k, \lambda(k))$. If $f_{j}(j=1,2)$ is sectionally analytic in $\mathbb{C}_{j}$ and rapidly decaying as $|k| \rightarrow \infty$ in the proper sheet, we have 


$$
\begin{gathered}
\left(P_{j}\left(f_{j}\right)\right)(k)=(-1)^{j-1} f_{j}(k), \quad k \in \mathbb{C}_{j}, \\
\left(P_{j}\left(f_{l}\right)\right)(k)=0, \quad k \in \mathbb{C}_{j} .
\end{gathered}
$$

We obtain

$$
\begin{array}{ll}
F^{-}(k)=\frac{1}{2 \pi i} \int_{\widetilde{\Sigma}} \frac{\lambda(k)+\lambda(\xi)}{2 \lambda(\xi)} \cdot \frac{F^{-}(\xi) g(\xi)}{\xi-k} d \xi, & k \in \mathbb{C}_{2}, \\
F^{+}(k)=\frac{1}{2 \pi i} \int_{\widetilde{\Sigma}} \frac{\lambda(k)+\lambda(\xi)}{2 \lambda(\xi)} \cdot \frac{F^{-}(\xi) g(\xi)}{\xi-k} d \xi, \quad k \in \mathbb{C}_{1} .
\end{array}
$$

For $\xi \in \widetilde{\Sigma}$, we can take limits from the proper sheet and connect by the analogue of Plemelj-Sokhotski formulas

$$
\begin{aligned}
& F^{+}\left(\xi_{0}\right)=\lim _{k \rightarrow \xi_{0} \in \widetilde{\Sigma}, k \in \mathbb{C}_{1}} \frac{1}{2 \pi i} \int_{\widetilde{\Sigma}} \frac{\lambda(k)+\lambda(\xi)}{2 \lambda(\xi)} \cdot \frac{F^{-}(\xi) g(\xi)}{\xi-k} d \xi \\
& F^{-}\left(\xi_{0}\right)=\lim _{k \rightarrow \xi_{0} \in \widetilde{\Sigma}, k \in \mathbb{C}_{2}} \frac{1}{2 \pi i} \int_{\widetilde{\Sigma}} \frac{\lambda(k)+\lambda(\xi)}{2 \lambda(\xi)} \cdot \frac{F^{-}(\xi) g(\xi)}{\xi-k} d \xi .
\end{aligned}
$$

In principle, the above $\mathrm{RH}$ problem can be analyzed for the two-sheeted problem. But a uniformizing coordinate makes the problem considerably more straight forward. This is discussed next.

\section{Uniformization coordinates}

Before discussing the properties of scattering data and solving the inverse problem, we introduce a uniformization variable $z$, see also Ref. 10, defined by the conformal mapping: $z=z(k)=k+$ $\lambda(k)$, where $\lambda=\sqrt{k^{2}+q_{0}^{2}}$ and the inverse mapping is given by $k=k(z)=\frac{1}{2}\left(z-\frac{q_{0}^{2}}{z}\right)$. Then $\lambda(z)=$ $\frac{1}{2}\left(z+\frac{q_{0}^{2}}{z}\right)$. We let $C_{0}$ be the circle of radius $q_{0}$ centered at the origin in the $z$-plane. We observe the following:

(1) The branch cut on either sheet is mapped onto $C_{0}$. In particular, $z\left( \pm i q_{0}\right)= \pm i q_{0}$ from either sheet, $z\left(0_{I}^{ \pm}\right)= \pm q_{0}$, and $z\left(0_{I I}^{ \pm}\right)=\mp q_{0}$.

(2) $\mathbb{K}_{1}$ is mapped onto the exterior of $C_{0}$ and $\mathbb{K}_{2}$ is mapped onto the interior of $C_{0}$. In particular, $z\left(\infty_{I}\right)=\infty$ and $z\left(\infty_{I I}\right)=0$; the first/second quadrants of $\mathbb{K}_{1}$ are mapped into the first/second quadrants outside $C_{0}$, respectively; the first/second quadrants of $\mathbb{K}_{2}$ are mapped into the second/first quadrants inside $C_{0}$, respectively; $z_{I} z_{I I}=q_{0}^{2}$.

(3) The regions in the $k$-plane such that $\mathfrak{J} \lambda>0$ and $\mathfrak{J} \lambda<0$ are mapped onto $D^{+}=\left\{z \in \mathbb{C}:\left(|z|^{2}-\right.\right.$ $\left.\left.q_{0}^{2}\right) \cdot \mathfrak{J} z>0\right\}$ and $D^{-}=\left\{z \in \mathbb{C}:\left(|z|^{2}-q_{0}^{2}\right) \cdot \mathfrak{I} z<0\right\}$, respectively.

From Theorem 4.3 and the definition of the uniformization variable $z$, we have that the eigenfunctions $M, N$ are analytic for $z \in D^{+}$and the eigenfunctions $\bar{M}, \bar{N}$ are analytic for in $z \in D^{-}$. See Figs. 5 and 6.

The contours (green) in two sheets [Figs. 3(a) and 3(b)] are mapped into Figs. 6(a) and 6(b), respectively, via the uniformization coordinate.

\section{E. Symmetries via uniformization coordinates}

It is found that $(1)$ when $z \rightarrow-z^{*}$, then $(k, \lambda) \rightarrow\left(-k^{*},-\lambda^{*}\right) ;(2)$ when $z \rightarrow-\frac{q_{0}^{2}}{z}$, then $(k, \lambda) \rightarrow(k,-\lambda)$. Hence, we have the same conclusions as in (3.48), and 


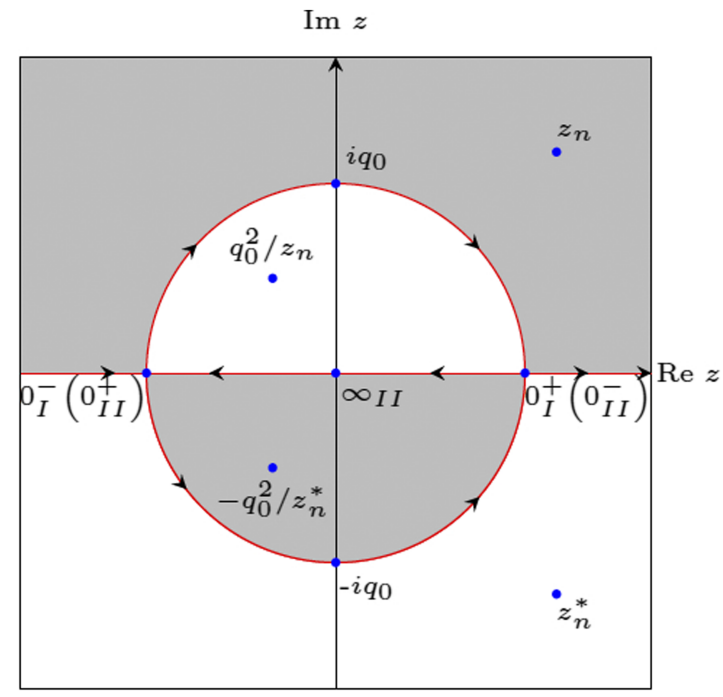

FIG. 5. The complex $z$-plane, showing the branch cut (red), the regions $D^{ \pm}$where $\mathfrak{I} \lambda>0$ (gray) and $\mathfrak{J} \lambda<0$ (white), respectively. In particular, the first sheet is mapped onto the region outside the circle, and the second sheet is mapped onto the region inside the circle. Importantly, the eigenfunctions $M, N$ are analytic for $z \in D^{+}$and the eigenfunctions $\bar{M}, \bar{N}$ are analytic for $z$ $\in D^{-}$

$$
\phi\left(x,-\frac{q_{0}^{2}}{z}\right)=\frac{\frac{q_{0}^{2}}{z}}{i \widetilde{q}} \cdot \bar{\phi}(x, z), \quad \psi\left(x,-\frac{q_{0}^{2}}{z}\right)=\frac{-i \widetilde{q}}{z} \cdot \bar{\psi}(x, z), \quad z \in D^{-} .
$$

Moreover,

$$
\begin{gathered}
a^{*}\left(-z^{*}\right)=a(z), \quad z \in D^{+}, \quad \bar{a}^{*}\left(-z^{*}\right)=\bar{a}(z), \quad z \in D^{-}, \quad b^{*}\left(-z^{*}\right)=-\bar{b}(z), \\
a\left(-\frac{q_{0}^{2}}{z}\right)=\bar{a}(z), \quad z \in D^{-}, \quad b\left(-\frac{q_{0}^{2}}{z}\right)=\frac{\widetilde{q}^{*}}{\widetilde{q}} \cdot \bar{b}(z) .
\end{gathered}
$$

Sheet I: (a)

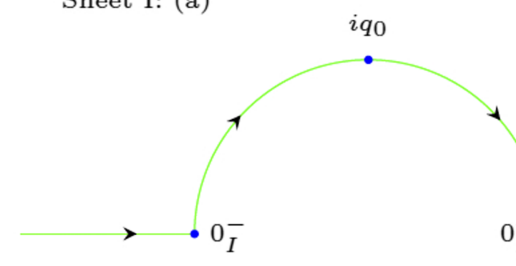

Sheet II: (b)

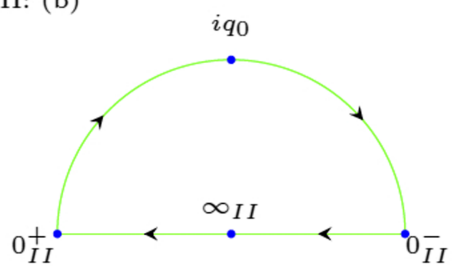

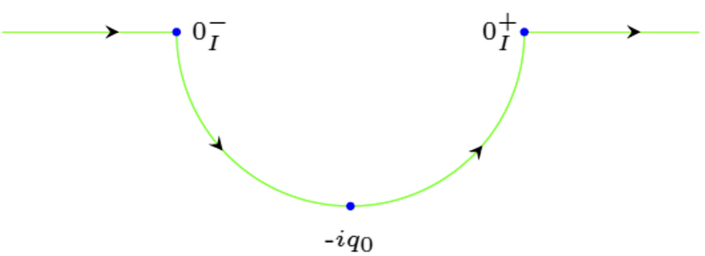

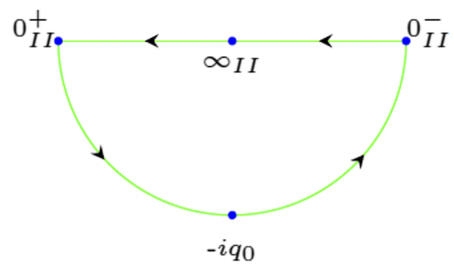

FIG. 6. (a) The contour (green) in the first sheet is mapped into the above curve in the $z$-plane. (b) The contour (green) in the second sheet is mapped into the above curve in the $z$-plane. 


\section{F. Asymptotic behavior of eigenfunctions and scattering data}

In order to solve the inverse problem, one has to determine the asymptotic behavior of eigenfunctions and scattering data both as $z \rightarrow \infty$ in $\mathbb{K}_{1}$ and as $z \rightarrow 0$ in $\mathbb{K}_{2}$. We have

$$
\begin{gathered}
N(x, z) \sim\left(\begin{array}{c}
-i q(x) \\
z
\end{array}\right), \quad z \rightarrow \infty, \quad N(x, z) \sim\left(\begin{array}{c}
-i \widetilde{q} \\
z \cdot \frac{q^{*}(-x)}{\widetilde{q}^{*}}
\end{array}\right), \quad z \rightarrow 0, \\
a(z)=\left\{\begin{array}{ll}
1, & z \rightarrow \infty, \\
1, & z \rightarrow 0,
\end{array} \quad \bar{a}(z)= \begin{cases}1, & z \rightarrow \infty, \\
1, & z \rightarrow 0,\end{cases} \right. \\
\lim _{z \rightarrow \infty} z b(z)=0, \quad \lim _{z \rightarrow 0} \frac{b(z)}{z^{2}}=0 .
\end{gathered}
$$

\section{G. Riemann-Hilbert problem via uniformization coordinates}

\section{Left scattering problem}

In order to take into account the behavior of the eigenfunctions, the "jump" conditions at $\Sigma$, where $\Sigma:=\left(-\infty,-q_{0}\right) \cup\left(q_{0},+\infty\right) \cup \overrightarrow{\left(q_{0},-q_{0}\right)} \cup\left\{q_{0} e^{i \theta}, \pi \leq \theta \leq 2 \pi\right\}_{\text {clockwise, upper circle }} \cup\left\{q_{0} e^{i \theta},-\pi \leq \theta \leq\right.$ $0\}_{\text {anticlockwise, lower circle }}$, can be written as

$$
\frac{M(x, z)}{z a(z)}-\frac{\bar{N}(x, z)}{z}=\rho(z) e^{i\left(z+\frac{q_{0}^{2}}{z}\right) x} \cdot \frac{N(x, z)}{z}, \quad \frac{\bar{M}(x, z)}{z \bar{a}(z)}-\frac{N(x, z)}{z}=\bar{\rho}(z) e^{-i\left(z+\frac{q_{0}^{2}}{z}\right) x} \cdot \frac{\bar{N}(x, z)}{z}
$$

so that the functions will be bounded at infinity, though having an additional pole at $z=0$. Note that $M(x, z) / a(z)$, as a function of $z$, is defined in $D^{+}$, where it has simple poles $z_{j}$, i.e., $a\left(z_{j}\right)=0$, and $\bar{M}(x, z) / \bar{a}(z)$ is defined in $D^{-}$, where it has simple poles $\bar{z}_{j}$, i.e., $\bar{a}\left(\bar{z}_{j}\right)=0$. It follows that

$$
M\left(x, z_{j}\right)=b\left(z_{j}\right) e^{i\left(z_{j}+\frac{q_{0}^{2}}{z_{j}}\right) x} \cdot N\left(x, z_{j}\right), \quad \bar{M}\left(x, \bar{z}_{j}\right)=\bar{b}\left(\bar{z}_{j}\right) e^{-i\left(\bar{z}_{j}+\frac{q_{0}^{2}}{\bar{z}_{j}}\right) x} \cdot \bar{N}\left(x, \bar{z}_{j}\right) .
$$

Then subtracting the values at infinity, the induced pole at the origin and the poles, assumed simple in $D^{+} / D^{-}$, respectively, at $a\left(z_{j}\right)=0, j=1,2, \ldots, J$ and $\bar{a}\left(\bar{z}_{j}\right), j=1,2, \ldots, \bar{J}$ give

$$
\begin{aligned}
& {\left[\frac{M(x, z)}{z a(z)}-\left(\begin{array}{l}
1 \\
0
\end{array}\right)-\frac{1}{z}\left(\begin{array}{c}
0 \\
-i \widetilde{q}^{*}
\end{array}\right)-\sum_{j=1}^{J} \frac{M\left(x, z_{j}\right)}{\left(z-z_{j}\right) z_{j} a^{\prime}\left(z_{j}\right)}\right]} \\
& -\left[\frac{\bar{N}(x, z)}{z}-\left(\begin{array}{l}
1 \\
0
\end{array}\right)-\frac{1}{z}\left(\begin{array}{c}
0 \\
-i \widetilde{q}^{*}
\end{array}\right)-\sum_{j=1}^{J} \frac{b\left(z_{j}\right) e^{i\left(z_{j}+\frac{q_{0}^{2}}{z_{j}}\right) x} \cdot N\left(x, z_{j}\right)}{\left(z-z_{j}\right) z_{j} a^{\prime}\left(z_{j}\right)}\right] \\
& =\rho(z) e^{i\left(z+\frac{q_{0}^{2}}{z}\right) x} \cdot \frac{N(x, z)}{z}, \\
& {\left[\frac{\bar{M}(x, z)}{z \bar{a}(z)}-\left(\begin{array}{l}
0 \\
1
\end{array}\right)-\frac{1}{z}\left(\begin{array}{c}
-\tilde{q} \widetilde{q} \\
0
\end{array}\right)-\sum_{j=1}^{\bar{J}} \frac{\bar{M}\left(x, \bar{z}_{j}\right)}{\left(z-\bar{z}_{j}\right) \bar{z}_{j} a^{\prime}\left(\bar{z}_{j}\right)}\right]} \\
& -\left[\frac{N(x, z)}{z}-\left(\begin{array}{l}
0 \\
1
\end{array}\right)-\frac{1}{z}\left(\begin{array}{c}
-i \widetilde{q} \\
0
\end{array}\right)-\sum_{j=1}^{\bar{J}} \frac{\bar{b}\left(\bar{z}_{j}\right) e^{-i\left(\bar{z}_{j}+\frac{q_{0}^{2}}{\bar{z}_{j}}\right) x} \cdot \bar{N}\left(x, \bar{z}_{j}\right)}{\left(z-\bar{z}_{j}\right) \bar{z}_{j} \bar{a}^{\prime}\left(\bar{z}_{j}\right)}\right] \\
& =\bar{\rho}(z) e^{-i\left(z+\frac{q_{0}^{2}}{z}\right) x} \cdot \frac{\bar{N}(x, z)}{z} \text {. }
\end{aligned}
$$


We now introduce the projection operators along $\Sigma$

$$
P_{ \pm}(f)(z)=\frac{1}{2 \pi i} \int_{\Sigma} \frac{f(\xi)}{\xi-(z \pm i 0)} d \xi,
$$

where $z$ lies in the \pm regions and $\Sigma:=\left(-\infty,-q_{0}\right) \cup\left(q_{0},+\infty\right) \cup \overrightarrow{\left(q_{0},-q_{0}\right)} \cup\left\{q_{0} e^{i \theta}, \pi \leq \theta\right.$ $\leq 2 \pi\}_{\text {clockwise, upper circle }} \cup\left\{q_{0} e^{i \theta},-\pi \leq \theta \leq 0\right\}_{\text {anticlockwise,lower circle }}$ indicated by the curve in Fig. 5 [red arrows with black heads].

If $f_{ \pm}(\xi)$ is analytic in $D^{ \pm}$and $f_{ \pm}(\xi)$ is decaying at large $\xi$, then

$$
P_{ \pm}\left(f_{ \pm}\right)(z)= \pm f_{ \pm}(z), \quad P_{\mp}\left(f_{ \pm}\right)(z)=0 .
$$

Applying $P_{-}$to (4.37) and $P_{+}$to (4.38), we can obtain

$$
\begin{aligned}
& \bar{N}(x, z)=\left(\begin{array}{c}
z \\
-i \widetilde{q}^{*}
\end{array}\right)+\sum_{j=1}^{J} \frac{z \cdot b\left(z_{j}\right) e^{i\left(z_{j}+\frac{q_{0}^{2}}{z_{j}}\right) x} \cdot N\left(x, z_{j}\right)}{\left(z-z_{j}\right) z_{j} a^{\prime}\left(z_{j}\right)}+\frac{z}{2 \pi i} \int_{\Sigma} \frac{\rho(\xi)}{\xi(\xi-z)} \cdot e^{i\left(\xi+\frac{q_{0}^{2}}{\xi}\right) x} \cdot N(x, \xi) d \xi, \\
& N(x, z)=\left(\begin{array}{c}
-i \widetilde{q} \\
z
\end{array}\right)+\sum_{j=1}^{\bar{J}} \frac{z \cdot \bar{b}\left(\bar{z}_{j}\right) e^{-i\left(\bar{z}_{j}+\frac{q_{0}^{2}}{\bar{z}_{j}}\right) x} \cdot \bar{N}\left(x, \bar{z}_{j}\right)}{\left(z-\bar{z}_{j}\right) \bar{z}_{j} \bar{a}^{\prime}\left(\bar{z}_{j}\right)}-\frac{z}{2 \pi i} \int_{\Sigma} \frac{\bar{\rho}(\xi)}{\xi(\xi-z)} \cdot e^{-i\left(\xi+\frac{q_{0}^{2}}{\xi}\right) x} \cdot \bar{N}(x, \xi) d \xi .
\end{aligned}
$$

\section{Right scattering problem}

As in Sec. III H 2, we can obtain $M(x, z)$ and $\bar{M}(x, z)$ by the right scattering problem,

$$
\begin{aligned}
& \bar{M}(x, z)=\left(\begin{array}{c}
-\widetilde{q} \\
z
\end{array}\right)+\sum_{j=1}^{J} \frac{-z \cdot \bar{b}\left(z_{j}\right) M\left(x, z_{j}\right) e^{-i\left(z_{j}+\frac{q_{0}^{2}}{z_{j}}\right) x}}{\left(z-z_{j}\right) z_{j} a^{\prime}\left(z_{j}\right)}+\frac{z}{2 \pi i} \int_{\Sigma} \frac{\rho^{*}\left(-\xi^{*}\right)}{\xi(\xi-z)} \cdot e^{-i\left(\xi+\frac{q_{0}^{2}}{\xi}\right) x} \cdot M(x, \xi) d \xi \\
& M(x, z)=\left(\begin{array}{c}
z \\
-\widetilde{q}^{*}
\end{array}\right)+\sum_{j=1}^{\bar{J}} \frac{-z \cdot b\left(\bar{z}_{j}\right) \bar{M}\left(x, \bar{z}_{j}\right) e^{i\left(\bar{z}_{j}+\frac{q_{0}^{2}}{\bar{z}_{j}}\right) x}}{\left(z-\bar{z}_{j}\right) \bar{z}_{j} \bar{a}^{\prime}\left(\bar{z}_{j}\right)}-\frac{z}{2 \pi i} \int_{\Sigma} \frac{\bar{\rho}^{*}\left(-\xi^{*}\right)}{\xi(\xi-z)} \cdot e^{i\left(\xi+\frac{q_{0}^{2}}{\xi}\right) x} \cdot \bar{M}(x, \xi) d \xi .
\end{aligned}
$$

\section{H. Recovery of the potentials}

Note that $N_{1}(x, z) \sim-i q(x)$ as $z \rightarrow \infty$ and

$$
N_{1}(x, z) \sim-\tilde{i q}+\sum_{j=1}^{\bar{J}} \frac{\bar{b}\left(\bar{z}_{j}\right) e^{-i\left(\bar{z}_{j}+\frac{q_{0}^{2}}{\bar{z}_{j}}\right) x} \cdot \bar{N}_{1}\left(x, \bar{z}_{j}\right)}{\bar{z}_{j} \bar{a}^{\prime}\left(\bar{z}_{j}\right)}+\frac{1}{2 \pi i} \int_{\Sigma} \frac{\bar{\rho}(\xi)}{\xi} \cdot e^{-i\left(\xi+\frac{q_{0}^{2}}{\xi}\right) x} \cdot \bar{N}_{1}(x, \xi) d \xi,
$$

we have

$$
q(x)=\widetilde{q}+i \sum_{j=1}^{\bar{J}} \frac{\bar{b}\left(\bar{z}_{j}\right) e^{-i\left(\bar{z}_{j}+\frac{q_{0}^{2}}{\bar{z}_{j}}\right) x} \cdot \bar{N}_{1}\left(x, \bar{z}_{j}\right)}{\bar{z}_{j} \bar{a}^{\prime}\left(\bar{z}_{j}\right)}+\frac{1}{2 \pi} \int_{\Sigma} \frac{\bar{\rho}(\xi)}{\xi} \cdot e^{-i\left(\xi+\frac{q_{0}^{2}}{\xi}\right) x} \cdot \bar{N}_{1}(x, \xi) d \xi .
$$

\section{Closing the system}

Similarly, we can get $J=\bar{J}$ from $a\left(-\frac{q_{0}^{2}}{z}\right)=\bar{a}(z)$. To close the system, by the symmetry relations between the eigenfunctions, we have 


$$
\begin{aligned}
& \left(\begin{array}{c}
\bar{M}_{1}(x, z) \\
\bar{M}_{2}(x, z)
\end{array}\right)=\left(\begin{array}{c}
-i \widetilde{q} \\
z
\end{array}\right)+\sum_{j=1}^{J} \frac{-z \cdot \bar{b}\left(z_{j}\right) e^{-i\left(z_{j}+\frac{q_{0}^{2}}{z_{j}}\right) x}}{\left(z-z_{j}\right) z_{j} a^{\prime}\left(z_{j}\right)} . \\
& \left(\begin{array}{c}
z_{j}+\sum_{l=1}^{J} \frac{-z_{j} \cdot b\left(\bar{z}_{l}\right) e^{i\left(\bar{z}_{l}+\frac{q_{0}^{2}}{\bar{z}_{l}}\right) x}}{\left(z_{j}-\bar{z}_{l}\right) \bar{z}_{l} \bar{a}^{\prime}\left(\bar{z}_{l}\right)} \cdot \bar{M}_{1}\left(x, \bar{z}_{l}\right)-\frac{z_{j}}{2 \pi i} \int_{\Sigma} \frac{\bar{\rho}^{*}\left(-\xi^{*}\right)}{\xi\left(\xi-z_{j}\right)} \cdot e^{i\left(\xi+\frac{q_{0}^{2}}{\xi}\right) x} \cdot \bar{M}_{1}(x, \xi) d \xi \\
-i \widetilde{q}^{*}+\sum_{l=1}^{J} \frac{-z_{j} \cdot b\left(\bar{z}_{l}\right) e^{i\left(\bar{z}_{l}+\frac{q_{0}^{2}}{\bar{z}_{l}}\right) x}}{\left(z_{j}-\bar{z}_{l}\right) \bar{z}_{l} \bar{a}^{\prime}\left(\bar{z}_{l}\right)} \cdot \bar{M}_{2}\left(x, \bar{z}_{l}\right)-\frac{z_{j}}{2 \pi i} \int_{\Sigma} \frac{\bar{\rho}^{*}\left(-\xi^{*}\right)}{\xi\left(\xi-z_{j}\right)} \cdot e^{i\left(\xi+\frac{q_{0}^{2}}{\xi}\right) x} \cdot \bar{M}_{2}(x, \xi) d \xi
\end{array}\right) \\
& +\frac{z}{2 \pi i} \int_{\Sigma} \frac{\rho^{*}\left(-\xi^{*}\right)}{\xi(\xi-z)} \cdot e^{-i\left(\xi+\frac{q_{0}^{2}}{\xi}\right) x} \\
& \left(\begin{array}{c}
\xi+\sum_{l=1}^{J} \frac{-\xi \cdot b\left(\bar{z}_{l}\right) e^{i\left(\bar{z}_{l}+\frac{q_{0}^{2}}{\bar{z}_{l}}\right) x}}{\left(\xi-\bar{z}_{l}\right) \bar{z}_{l} \bar{a}^{\prime}\left(\bar{z}_{l}\right)} \cdot \bar{M}_{1}\left(x, \bar{z}_{l}\right)-\frac{\xi}{2 \pi i} \int_{\Sigma} \frac{\bar{\rho}^{*}\left(-\eta^{*}\right)}{\eta(\eta-\xi)} \cdot e^{i\left(\eta+\frac{q_{0}^{2}}{\eta}\right) x} \cdot \bar{M}_{1}(x, \eta) d \eta \\
-i \widetilde{q}^{*}+\sum_{l=1}^{J} \frac{-\xi \cdot b\left(\bar{z}_{l}\right) e^{i\left(\bar{z}_{l}+\frac{q_{0}^{2}}{\bar{z}_{l}}\right) x}}{\left(\xi-\bar{z}_{l}\right) \bar{z}_{l} \bar{a}^{\prime}\left(\bar{z}_{l}\right)} \cdot \bar{M}_{2}\left(x, \bar{z}_{l}\right)-\frac{\xi}{2 \pi i} \int_{\Sigma} \frac{\bar{\rho}^{*}\left(-\eta^{*}\right)}{\eta(\eta-\xi)} \cdot e^{i\left(\eta+\frac{q_{0}^{2}}{\eta}\right) x} \cdot \bar{M}_{2}(x, \eta) d \eta
\end{array}\right) d \xi .
\end{aligned}
$$

We note that from Eq. (4.46), $q(x)$ is given only in terms of the component $\bar{N}_{1}$. We can use only the second component of Eq. (4.47) to find $\bar{M}_{2}$ which via the symmetry relation (3.50) yields this function and hence $q(x)$. Hence to complete the inverse scattering, we reduce the problem to solving an integral equation in terms of the component $\bar{M}_{2}$ only.

\section{J. Trace formula}

In a similar manner to Sec. III K, we can get the trace formula as follows:

$$
\begin{aligned}
& \log a(z)=\log \left(\prod_{j=1}^{J_{1}} \frac{z-z_{j}}{z+\frac{q_{0}^{2}}{z_{j}}} \cdot \frac{z+z_{j}^{*}}{z-\frac{q_{0}^{2}}{z_{j}^{*}}} \cdot \prod_{i=1}^{J_{2}} \frac{z-\widetilde{z}_{i}}{z+\frac{q_{0}^{2}}{\widetilde{z}_{i}}}\right)+\frac{1}{2 \pi i} \int_{\Sigma} \frac{\log \left(1-b(\xi) b^{*}\left(-\xi^{*}\right)\right)}{\xi-z} d \xi, \quad z \in D^{+}, \\
& \log \bar{a}(z)=\log \left(\prod_{j=1}^{J_{1}} \frac{z+\frac{q_{0}^{2}}{z_{j}}}{z-z_{j}} \cdot \frac{z-\frac{q_{0}^{2}}{z_{j}^{*}}}{z+z_{j}^{*}} \cdot \prod_{i=1}^{J_{2}} \frac{z+\frac{q_{0}^{2}}{\widetilde{z}_{i}}}{z-\widetilde{z}_{i}}\right)-\frac{1}{2 \pi i} \int_{\Sigma} \frac{\log \left(1-b(\xi) b^{*}\left(-\xi^{*}\right)\right)}{\xi-z} d \xi, \quad z \in D^{-},
\end{aligned}
$$

where $\mathfrak{R} \widetilde{z}_{i}=0, \mathfrak{R} z_{j} \neq 0$, and $2 J_{1}+J_{2}=J$.

\section{K. Reflectioness potentials and soliton solutions}

Reflectioness potentials and soliton solutions correspond to zero reflection coefficients, i.e., $\rho(\xi)$ $=0$ and $\bar{\rho}(\xi)=0$ for all $\xi \in \Sigma$. We also note that from the symmetry relation $\bar{b}(z)=-b^{*}\left(-z^{*}\right)$, it follows that the reflection coefficients $\rho(z)=b(z) / a(z), \bar{\rho}(z)=\bar{b}(z) / \bar{a}(z)$ will both vanish when $b(z)$ $=0$ for $z$ on $\Sigma$. By substituting $z=\bar{z}_{l}$ in (4.47), the system (4.47) reduces to algebraic systems of equations that determine the functional form of these special potentials. When time dependence is added, the reflectionless potentials correspond to soliton solutions. The reduced equation takes the form 


$$
\left(\begin{array}{c}
\bar{M}_{1}\left(x, \bar{z}_{l}\right) \\
\bar{M}_{2}\left(x, \bar{z}_{l}\right)
\end{array}\right)=\left(\begin{array}{c}
-\tilde{i} \widetilde{q} \\
\bar{z}_{l}
\end{array}\right)+\sum_{j=1}^{J} \frac{-\bar{z}_{l} \cdot \bar{b}\left(z_{j}\right) e^{-i\left(z_{j}+\frac{q_{0}^{2}}{z_{j}}\right) x}}{\left(\bar{z}_{l}-z_{j}\right) z_{j} a^{\prime}\left(z_{j}\right)}\left(\begin{array}{c}
z_{j}+\sum_{l=1}^{J} \frac{-z_{j} \cdot b\left(\bar{z}_{l}\right) e^{i\left(\bar{z}_{l}+\frac{q_{0}^{2}}{\bar{z}_{l}}\right) x}}{\left(z_{j}-\bar{z}_{l}\right) \bar{z}_{l} \bar{a}^{\prime}\left(\bar{z}_{l}\right)} \cdot \bar{M}_{1}\left(x, \bar{z}_{l}\right) \\
-i \widetilde{q}^{*}+\sum_{l=1}^{J} \frac{-z_{j} \cdot b\left(\bar{z}_{l}\right) e^{i\left(\bar{z}_{l}+\frac{q_{0}^{2}}{\bar{z}_{l}}\right) x}}{\left(z_{j}-\bar{z}_{l}\right) \bar{z}_{l} \bar{a}^{\prime}\left(\bar{z}_{l}\right)} \cdot \bar{M}_{2}\left(x, \bar{z}_{l}\right)
\end{array}\right) .
$$

The above equations are an algebraic system to solve for either $N\left(x, z_{l}\right)$ or $\bar{M}\left(x, \bar{z}_{l}\right), l=1,2, \ldots, J$. The potential is reconstructed from Eq. (4.46) with $\rho(\xi)=0, \bar{\rho}(\xi)=0$, i.e.,

$$
q(x)=\widetilde{q}+i \sum_{j=1}^{J} \frac{\bar{b}\left(\bar{z}_{j}\right) e^{-i\left(\bar{z}_{j}+\frac{q_{0}^{2}}{\bar{z}_{j}}\right) x} \cdot \bar{N}_{1}\left(x, \bar{z}_{j}\right)}{\bar{z}_{j} \bar{a}^{\prime}\left(\bar{z}_{j}\right)} .
$$

Since $a(z) \sim 1$ as $z \rightarrow 0$, from the trace formula when $b(\xi)=0$ in $\Sigma$, we have the following constraint for the reflectionless potentials:

$$
\prod_{j=1}^{J_{1}} \frac{\left|z_{j}\right|^{4}}{q_{0}^{4}} \cdot \prod_{i=1}^{J_{2}}\left(\frac{\left|\widetilde{z}_{i}\right|^{2}}{q_{0}^{2}}\right)=1,
$$

where $2 J_{1}+J_{2}=J$. We claim that $J \geq 2$. Otherwise, if $J=1$, then $J_{1}=0$ and $J_{2}=1$, which implies that $\left|\widetilde{z}_{1}\right|^{2}=q_{0}^{2}$; hence, the eigenvalue lies on the circle, which in this case is the continuous spectrum. Such eigenvalues are not proper; they are not considered here.

\section{Discrete scattering data and their symmetries}

In order to find reflectionless potentials and solitons, we need to be able to calculate the relevant discrete scattering data. The coefficients $b\left(z_{j}\right)$ and $\bar{b}\left(\bar{z}_{j}\right), j=1,2, \ldots, J$, can be calculated in the same way as in Sec. III L to find

$$
b\left(z_{j}\right) b^{*}\left(-z_{j}^{*}\right)=1, \quad \bar{b}\left(\bar{z}_{j}\right) \bar{b}^{*}\left(-\bar{z}_{j}^{*}\right)=1, \quad-b\left(z_{j}\right) \bar{b}\left(z_{j}\right)=1, \quad-b\left(\bar{z}_{j}\right) \bar{b}\left(\bar{z}_{j}\right)=1 .
$$

The functions $a^{\prime}\left(z_{j}\right), \bar{a}^{\prime}\left(z_{j}\right)$ can be calculated via the trace formulae.

\section{Reflectionless potential solution: 2-Eigenvalue}

In this subsection, we construct an explicit 2-eigenvalue reflectionless potential by setting $J=2$; this solution turns into a soliton solution when time dependence is added. The simplest reflectionless potential case obtains when $J_{1}=0$ and $J_{2}=2$; as mentioned above, when $J_{1}=1, J_{2}=0$ leads to eigenvalues on the continuous spectrum. Note that $\left|\widetilde{z}_{1}\right| \cdot\left|\widetilde{z}_{2}\right|=q_{0}^{2}$. Now let $\widetilde{z}_{1}=i v_{1}$, where $v_{1}>q_{0}$. Then $\widetilde{z}_{2}=-i \frac{q_{0}^{2}}{v_{1}}, a\left(i v_{1}\right)=a\left(-i \frac{q_{0}^{2}}{v_{1}}\right)=0$, and $\bar{a}\left(i \frac{q_{0}^{2}}{v_{1}}\right)=\bar{a}\left(-i v_{1}\right)=0$. Thus,

$$
a(z)=\frac{z-i v_{1}}{z-i \frac{q_{0}^{2}}{v_{1}}} \cdot \frac{z+i \frac{q_{0}^{2}}{v_{1}}}{z+i v_{1}}, \quad \bar{a}(z)=\frac{z-i \frac{q_{0}^{2}}{v_{1}}}{z-i v_{1}} \cdot \frac{z+i v_{1}}{z+i \frac{q_{0}^{2}}{v_{1}}},
$$

we can get

$$
\begin{aligned}
& a^{\prime}\left(i v_{1}\right)=\frac{i\left(v_{1}+\frac{q_{0}^{2}}{v_{1}}\right)}{2\left(q_{0}^{2}-v_{1}^{2}\right)}, \quad a^{\prime}\left(-i \frac{q_{0}^{2}}{v_{1}}\right)=\frac{i\left(\frac{q_{0}^{2}}{v_{1}}+v_{1}\right)}{2 q_{0}^{2}\left(\frac{q_{0}^{2}}{v_{1}^{2}}-1\right)}, \\
& \bar{a}^{\prime}\left(-i v_{1}\right)=\frac{i\left(v_{1}+\frac{q_{0}^{2}}{v_{1}}\right)}{2\left(v_{1}^{2}-q_{0}^{2}\right)}, \quad \bar{a}^{\prime}\left(i \frac{q_{0}^{2}}{v_{1}}\right)=\frac{-i\left(v_{1}+\frac{q_{0}^{2}}{v_{1}}\right)}{2 q_{0}^{2}\left(\frac{q_{0}^{2}}{v_{1}^{2}}-1\right)} .
\end{aligned}
$$


Similarly, from Sec. IV L, we can get $\left|b\left(i v_{1}\right)\right|=\left|\bar{b}\left(i v_{1}\right)\right|=\left|b\left(-i \frac{q_{0}^{2}}{v_{1}}\right)\right|=\left|\bar{b}\left(-i \frac{q_{0}^{2}}{v_{1}}\right)\right|=\left|b\left(-i v_{1}\right)\right|=\left|\bar{b}\left(-i v_{1}\right)\right|$ $=\left|b\left(i \frac{q_{0}^{2}}{v_{1}}\right)\right|=\left|\bar{b}\left(i \frac{q_{0}^{2}}{v_{1}}\right)\right|=1$. So we can write $b\left(i v_{1}\right)=e^{i \varphi_{1}}$ and $b\left(-i \frac{q_{0}^{2}}{v_{1}}\right)=e^{i \varphi_{2}}$. By the symmetry relations $b^{*}\left(-z^{*}\right)=-\bar{b}(z)$ and $b\left(-\frac{q_{0}^{2}}{z}\right)=\frac{\widetilde{q}^{*}}{\bar{q}} \bar{b}(z)$, we can obtain $\bar{b}\left(i \frac{q_{0}^{2}}{v_{1}}\right)=e^{i\left(2 \theta+\varphi_{1}\right)}, \bar{b}\left(-i v_{1}\right)$ $=e^{i\left(2 \theta+\varphi_{2}\right)}, \bar{b}\left(i v_{1}\right)=-e^{-i \varphi_{1}}, \bar{b}\left(-i \frac{q_{0}^{2}}{v_{1}}\right)=-e^{-i \varphi_{2}}, b\left(-i v_{1}\right)=-e^{-i\left(2 \theta+\varphi_{2}\right)}$, and $b\left(i \frac{q_{0}^{2}}{v_{1}}\right)=-e^{-i\left(2 \theta+\varphi_{1}\right)}$. Hence, we have

$$
\begin{aligned}
& \bar{M}_{2}^{*}\left(-x, i \frac{q_{0}^{2}}{v_{1}}\right)=\frac{i q_{0} e^{2 v_{1} x} \cdot\left(q_{0}^{2}+v_{1}^{2}\right) \cdot\left(q_{0}+v_{1} e^{\left(\frac{q_{0}^{2}}{v_{1}}-v_{1}\right) x} e^{i\left(\theta+\varphi_{2}\right)}\right)}{v_{1}\left[2 q_{0} v_{1} \cdot e^{\left(\frac{q_{0}^{2}}{v_{1}}+v_{1}\right) x} \cdot e^{i \theta}\left(e^{i \varphi_{1}}-e^{i \varphi_{2}}\right)-\left(q_{0}^{2}+v_{1}^{2}\right)\left(e^{2 v_{1} x}-e^{\frac{2 q_{0}^{2}}{v_{1}} x} \cdot e^{i\left(2 \theta+\varphi_{1}+\varphi_{2}\right)}\right)\right]}, \\
& \bar{M}_{2}^{*}\left(-x,-i v_{1}\right)=\frac{i e^{v_{1} x} \cdot\left(q_{0}^{2}+v_{1}^{2}\right) \cdot\left(-q_{0} e^{\frac{q_{0}^{2}}{v_{1}} x} e^{i\left(\theta+\varphi_{1}\right)}+v_{1} e^{v_{1} x}\right)}{2 q_{0} v_{1} \cdot e^{\left(\frac{q_{0}^{2}}{v_{1}}+v_{1}\right) x} \cdot e^{i \theta}\left(e^{i \varphi_{2}}-e^{i \varphi_{1}}\right)-\left(q_{0}^{2}+v_{1}^{2}\right)\left(e^{2 v_{1} x}-e^{\frac{2 q_{0}^{2}}{v_{1}} x} \cdot e^{i\left(2 \theta+\varphi_{1}+\varphi_{2}\right)}\right)}
\end{aligned}
$$

By (4.51), we have

$$
q(x)=\widetilde{q}-i \frac{2\left(\frac{q_{0}^{2}}{v_{1}^{2}}-1\right) e^{i\left(2 \theta+\varphi_{1}\right)} e^{\left(\frac{q_{0}^{2}}{v_{1}}-v_{1}\right) x}}{1+\frac{q_{0}^{2}}{v_{1}^{2}}} \bar{M}_{2}^{*}\left(-x, i \frac{q_{0}^{2}}{v_{1}}\right)-i \frac{2\left(v_{1}-\frac{q_{0}^{2}}{v_{1}}\right) e^{i\left(2 \theta+\varphi_{2}\right)} e^{\left(\frac{q_{0}^{2}}{v_{1}}-v_{1}\right) x}}{\frac{q_{0}^{2}}{v_{1}}+v_{1}} \bar{M}_{2}^{*}\left(-x,-i v_{1}\right) .
$$

\section{N. Time evolution}

As in Sec. III O, we find

$$
\frac{\partial a(t)}{\partial t}=0, \quad \frac{\partial \bar{a}(t)}{\partial t}=0, \quad \frac{\partial b(t)}{\partial t}=2 i\left(q_{0}^{2}-2 \lambda k\right) b(t) .
$$

Hence, $a(t)$ and $\bar{a}(t)$ are time independent. Moreover,

$$
b\left(i v_{1}, t\right)=e^{i \varphi_{1}} \cdot e^{2 i\left[q_{0}^{2}+\frac{1}{2}\left(v_{1}^{2}-\frac{q_{0}^{4}}{v_{1}^{2}}\right)\right] t}, \quad b\left(-i \frac{q_{0}^{2}}{v_{1}}, t\right)=e^{i \varphi_{2}} \cdot e^{2 i\left[q_{0}^{2}-\frac{1}{2}\left(v_{1}^{2}-\frac{q_{0}^{4}}{v_{1}^{2}}\right)\right] t} .
$$

Putting all the above into the formula we had for the reflectionless potential, we obtain the following 2-soliton solution, which is oscillating in time or "breathing" soliton solution:

$$
\begin{aligned}
& q(x, t)=\left(e ^ { - \frac { i } { 2 } ( \frac { 2 ( q _ { 0 } ^ { 2 } + v _ { 1 } ^ { 2 } ) ( t ( q _ { 0 } ^ { 2 } + v _ { 1 } ^ { 2 } ) - i v _ { 1 } x ) } { v _ { 1 } ^ { 2 } } + \varphi _ { 1 } + \varphi _ { 2 } ) } \cdot \left(2 q_{0}^{4} \cdot e^{2 i t v_{1}^{2}+\left(\frac{q_{0}^{2}}{v_{1}}+v_{1}\right) x+i\left(\theta+\varphi_{1}\right)}-2 v_{1}^{4} e^{\frac{2 i q_{0}^{4} t}{v_{1}^{2}}+\left(\frac{q_{0}^{2}}{v_{1}}+v_{1}\right) x+i\left(\theta+\varphi_{2}\right)}\right.\right. \\
& \left.\left.-q_{0} v_{1}\left(q_{0}^{2}+v_{1}^{2}\right) \cdot e^{\frac{i t\left(q_{0}^{4}+v_{1}^{4}\right)}{v_{1}^{2}}+2 v_{1} x}+q_{0} v_{1}\left(q_{0}^{2}+v_{1}^{2}\right) \cdot e^{i \cdot \frac{q_{0}^{4} t-2 i q_{0}^{2} v_{1} x+v_{1}^{2}\left(t v_{1}^{2}+2 \theta+\varphi_{1}+\varphi_{2}\right)}{v_{1}^{2}}}\right)\right) / \\
& \left(2 v_{1}\left(-2 i q_{0} v_{1} \sin \left[\frac{1}{2}\left(2 t\left(\frac{q_{0}^{4}}{v_{1}^{2}}-v_{1}^{2}\right)-\varphi_{1}+\varphi_{2}\right)\right]\right)+\left(q_{0}^{2}+v_{1}^{2}\right) \sinh \left[\frac{q_{0}^{2} x}{v_{1}}+\frac{-2 v_{1} x+i\left(2 \theta+\varphi_{1}+\varphi_{2}\right)}{2}\right]\right) \text {. }
\end{aligned}
$$

A typical breather solution is plotted in Fig. 7. 


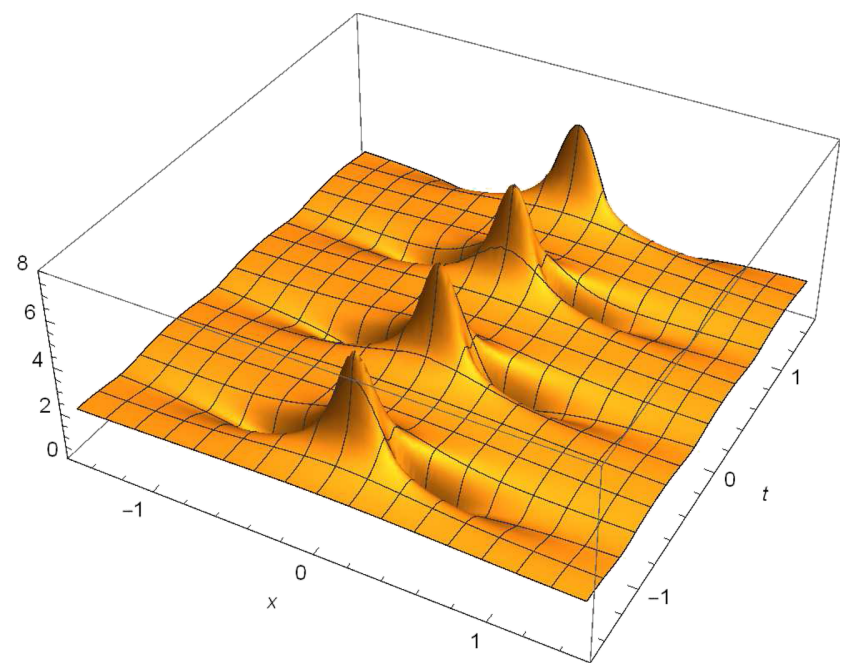

FIG. 7. The amplitude of $q(x, t)$ with $\theta=\frac{\pi}{2}, \varphi_{1}=0, \varphi_{2}=0, v_{1}=3$, and $q_{0}=2$.

\section{THE CASE OF $\sigma=1$ WITH $\Delta \theta=\theta_{+}-\theta_{-}=0$}

Next we will study the nonlocal nonlinear Schrödinger (NLS) equation (1.1) with $\sigma=1$,

$$
i q_{t}(x, t)=q_{x x}(x, t)-2 q^{2}(x, t) q^{*}(-x, t)
$$

with nonzero boundary conditions (NZBCs)

$$
q(x, t) \rightarrow \widehat{q}_{ \pm}(t):=\widehat{q}(t)=q_{0} e^{2 i q_{0}^{2} t+i \theta} \text {, as } x \rightarrow \pm \infty,
$$

where $q_{0}>0,0 \leq \theta<2 \pi$. Note we take $\theta_{+}=\theta_{-}=\theta$.

\section{A. Direct scattering}

With this condition, Eq. (2.13) conveniently reduces to

$$
\frac{\partial^{2} v_{j}}{\partial x^{2}}=-\left(k^{2}-q_{0}^{2}\right) v_{j}, \quad j=1,2
$$

Each of the two equations has two linearly independent solutions $e^{i \lambda x}$ and $e^{-i \lambda x}$ as $|x| \rightarrow \infty$, where $\lambda=\sqrt{k^{2}-q_{0}^{2}}$. The variable $k$ is then thought of as belonging to a Riemann surface $\mathbb{K}$ consisting of two sheets $\mathbb{C}_{1}$ and $\mathbb{C}_{2}$ with both coinciding with the complex plane cut along $\left(-\infty,-q_{0}\right] \cup\left[q_{0},+\infty\right)$ with its edges glued in such a way that $\lambda(k)$ is continuous through the cut. The Riemann surface and the definition of $\lambda$ are the same as discussed in Sec. III $(\sigma=-1, \Delta \theta=\pi$.)

\section{B. Eigenfunctions}

Following the approach discussed in Sec. III, we introduce the eigenfunctions defined by the following boundary conditions:

$$
\begin{aligned}
& \phi(x, k) \sim w e^{-i \lambda x}, \quad \bar{\phi}(x, k) \sim \bar{w} e^{i \lambda x} \text { as } x \rightarrow-\infty, \\
& \psi(x, k) \sim v e^{i \lambda x}, \quad \bar{\psi}(x, k) \sim \bar{v} e^{-i \lambda x} \text { as } \quad x \rightarrow+\infty,
\end{aligned}
$$

where

$$
w=\left(\begin{array}{c}
\lambda+k \\
i \widehat{q}^{*}
\end{array}\right), \quad \bar{w}=\left(\begin{array}{c}
-\hat{i} \widehat{q} \\
\lambda+k
\end{array}\right), \quad v=\left(\begin{array}{c}
-i \widehat{q} \\
\lambda+k
\end{array}\right), \quad \bar{v}=\left(\begin{array}{c}
\lambda+k \\
i \widehat{q}^{*}
\end{array}\right)
$$


satisfy the boundary conditions; they are not unique. As in Sec. III, we consider functions with constant boundary conditions and define the same bounded eigenfunctions $M(x, k), N(x, k), \bar{M}(x, k), \bar{N}(x, k)$ except we use the boundary conditions defined in (5.6). The bounded eigenfunctions can be represented by means of integral equations, the formulae are the same as in (3.9)-(3.12) except for the constant terms, which are defined in (5.6).

\section{Symmetry reductions}

Taking into account boundary conditions, we can obtain

$$
\psi(x, k)=\left(\begin{array}{cc}
0 & 1 \\
-1 & 0
\end{array}\right) \phi^{*}\left(-x,-k^{*}\right), \quad \bar{\psi}(x, k)=\left(\begin{array}{cc}
0 & -1 \\
1 & 0
\end{array}\right) \bar{\phi}^{*}\left(-x,-k^{*}\right) .
$$

The scattering relations are the same as in (3.41)-(3.45) except for the relation between $b$ and $\bar{b}$, i.e., $b^{*}\left(-k^{*}\right)=\bar{b}(k)$.

Using the notation $\lambda=\lambda^{+}=-\lambda^{-}$, we have the following symmetry:

$$
\phi^{\mp}(x, k)=\frac{\lambda^{\mp}+k}{-i \widehat{q}} \bar{\phi}^{ \pm}(x, k), \quad \psi^{\mp}(x, k)=\frac{\lambda^{\mp}+k}{i \widehat{q}^{*}} \bar{\psi}^{ \pm}(x, k)
$$

for $k \in\left(-\infty,-q_{0}\right] \cup\left[q_{0},+\infty\right)$. Moreover,

$$
a^{ \pm}(k)=\bar{a}^{\mp}(k), \quad b^{ \pm}(k)=-\frac{\widehat{q}^{*}}{\widehat{q}} \cdot \bar{b}^{\mp}(k)
$$

for $k \in\left(-\infty,-q_{0}\right] \cup\left[q_{0},+\infty\right)$

\section{Uniformization coordinates}

As in Sec. III, we introduce a uniformization variable $z$, defined by the conformal mapping: $z$ $=z(k)=k+\lambda(k)$, and the inverse mapping is given by $k=k(z)=\frac{1}{2}\left(z+\frac{q_{0}^{2}}{z}\right)$. Then $\lambda(z)=\frac{1}{2}\left(z-\frac{q_{0}^{2}}{z}\right)$.

\section{E. Symmetries via uniformization coordinates}

We have seen that (1) when $z \rightarrow-z^{*}$, then $(k, \lambda) \rightarrow\left(-k^{*},-\lambda^{*}\right)$ and (2) when $z \rightarrow \frac{q_{0}^{2}}{z}$, then $(k, \lambda)$ $\rightarrow(k,-\lambda)$. Hence,

$$
\begin{gathered}
\psi(x, z)=\left(\begin{array}{cc}
0 & 1 \\
-1 & 0
\end{array}\right) \phi^{*}\left(-x,-z^{*}\right), \quad \bar{\psi}(x, z)=\left(\begin{array}{cc}
0 & -1 \\
1 & 0
\end{array}\right) \bar{\phi}^{*}\left(-x,-z^{*}\right), \\
\phi\left(x, \frac{q_{0}^{2}}{z}\right)=\frac{\frac{q_{0}^{2}}{z}}{-i \widehat{q}} \bar{\phi}(x, z), \quad \psi\left(x, \frac{q_{0}^{2}}{z}\right)=\frac{-\hat{\imath} \widehat{q}}{z} \bar{\psi}(x, z), \quad \mathfrak{J} z<0 .
\end{gathered}
$$

Moreover,

$$
\begin{gathered}
a^{*}\left(-z^{*}\right)=a(z), \quad \bar{a}^{*}\left(-z^{*}\right)=\bar{a}(z), \quad b^{*}\left(-z^{*}\right)=\bar{b}(z), \\
a\left(\frac{q_{0}^{2}}{z}\right)=\bar{a}(z), \quad \mathfrak{J} z<0, \quad b\left(\frac{q_{0}^{2}}{z}\right)=-\frac{\widehat{q}^{*}}{\widehat{q}} \cdot \bar{b}(z) .
\end{gathered}
$$

\section{F. Asymptotic behavior of eigenfunctions and scattering data}

In order to solve the inverse problem, one has to determine the asymptotic behavior of eigenfunctions and scattering data both as $z \rightarrow \infty$ and as $z \rightarrow 0$. We have

$$
\begin{gathered}
\bar{N}(x, z) \sim\left(\begin{array}{c}
z \\
i q^{*}(-x)
\end{array}\right), \quad z \rightarrow \infty, \quad \bar{N}(x, z) \sim\left(\begin{array}{c}
z \cdot \frac{q(x)}{\widehat{q}^{*}} \\
i \widehat{q}^{*}
\end{array}\right), \quad z \rightarrow 0, \\
a(z)=\left\{\begin{array}{ll}
1, & z \rightarrow \infty, \\
1, & z \rightarrow 0,
\end{array} \quad \bar{a}(z)= \begin{cases}1, & z \rightarrow \infty, \\
1, & z \rightarrow 0,\end{cases} \right.
\end{gathered}
$$




$$
\lim _{z \rightarrow \infty} z b(z)=0, \quad \lim _{z \rightarrow 0} \frac{b(z)}{z^{2}}=0 .
$$

\section{G. Left and right scattering problems}

Using similar methods as in Sec. III, we find $\bar{N}(x, z), N(x, z), \bar{M}(x, z)$, and $M(x, z)$. They are almost the same as (3.64), (3.65), (3.75), and (3.76). The only differences are the constant terms, which are shown in (5.6).

\section{H. Recovery of the potentials}

Note that $\frac{\bar{N}_{1}(x, z)}{z} \sim \frac{q(x)}{\widehat{q}}$ as $z \rightarrow 0$ and

$$
\frac{\bar{N}_{1}(x, z)}{z} \rightarrow 1+\sum_{j=1}^{J} \frac{b\left(z_{j}\right) e^{i\left(z_{j}-\frac{q_{0}^{2}}{z_{j}}\right) x}}{-z_{j}^{2} a^{\prime}\left(z_{j}\right)} \cdot N_{1}\left(x, z_{j}\right)+\frac{1}{2 \pi i} \int_{-\infty}^{+\infty} \frac{\rho(\xi)}{\xi^{2}} \cdot e^{i\left(\xi-\frac{q_{0}^{2}}{\xi}\right) x} \cdot N_{1}(x, \xi) d \xi
$$

as $z \rightarrow 0$, therefore,

$$
q(z)=\widehat{q} \cdot\left[1+\sum_{j=1}^{J} \frac{b\left(z_{j}\right) e^{i\left(z_{j}-\frac{q_{0}^{2}}{z_{j}}\right) x}}{-z_{j}^{2} a^{\prime}\left(z_{j}\right)} \cdot N_{1}\left(x, z_{j}\right)+\frac{1}{2 \pi i} \int_{-\infty}^{+\infty} \frac{\rho(\xi)}{\xi^{2}} \cdot e^{i\left(\xi-\frac{q_{0}^{2}}{\xi}\right) x} \cdot N_{1}(x, \xi) d \xi\right] .
$$

\section{Closing the system}

We find $J=\bar{J}$ from $a\left(\frac{q_{0}^{2}}{z}\right)=\bar{a}(z)$. To close the system, by the symmetry relations between the eigenfunctions, we have

$$
\begin{aligned}
& \left(\begin{array}{l}
N_{1}(x, z) \\
N_{2}(x, z)
\end{array}\right)=\left(\begin{array}{c}
-\hat{q} \widehat{q} \\
z
\end{array}\right)+\sum_{j=1}^{J} \frac{z \cdot \bar{b}\left(\bar{z}_{j}\right) e^{-i\left(\bar{z}_{j}-\frac{q_{0}^{2}}{\bar{z}_{j}}\right) x}}{\left(z-\bar{z}_{j}\right) \bar{z}_{j} \bar{a}^{\prime}\left(\bar{z}_{j}\right)} \cdot \\
& \left(\begin{array}{l}
\bar{z}_{j}+\sum_{l=1}^{J} \frac{\bar{z}_{j} \cdot b\left(z_{l}\right) e^{i\left(z_{l}-\frac{q_{0}^{2}}{z_{l}}\right) x}}{\left(\bar{z}_{j}-z_{l}\right) z_{l} a^{\prime}\left(z_{l}\right)} \cdot N_{1}\left(x, z_{l}\right)+\frac{\bar{z}_{j}}{2 \pi i} \int_{-\infty}^{+\infty} \frac{\rho(\xi)}{\xi\left(\xi-\bar{z}_{j}\right)} \cdot e^{i\left(\xi-\frac{q_{0}^{2}}{\xi}\right) x} \cdot N_{1}(x, \xi) d \xi \\
\widehat{q}^{*}+\sum_{l=1}^{J} \frac{\bar{z}_{j} \cdot b\left(z_{l}\right) e^{i\left(z_{l}-\frac{q_{0}^{2}}{z_{l}}\right) x}}{\left(\bar{z}_{j}-z_{l}\right) z_{l} a^{\prime}\left(z_{l}\right)} \cdot N_{2}\left(x, z_{l}\right)+\frac{\bar{z}_{j}}{2 \pi i} \int_{-\infty}^{+\infty} \frac{\rho(\xi)}{\xi\left(\xi-\bar{z}_{j}\right)} \cdot e^{i\left(\xi-\frac{q_{0}^{2}}{\xi}\right) x} \cdot N_{2}(x, \xi) d \xi
\end{array}\right) \\
& -\frac{z}{2 \pi i} \int_{-\infty}^{+\infty} \frac{\bar{\rho}(\xi)}{\xi(\xi-z)} \cdot e^{-i\left(\xi-\frac{q_{0}^{2}}{\xi}\right) x} \cdot \\
& \left(\begin{array}{l}
\xi+\sum_{l=1}^{J} \frac{\xi \cdot b\left(z_{l}\right) e^{i\left(z_{l}-\frac{q_{0}^{2}}{z_{l}}\right) x}}{\left(\xi-z_{l}\right) z_{l} a^{\prime}\left(z_{l}\right)} \cdot N_{1}\left(x, z_{l}\right)+\frac{\xi}{2 \pi i} \int_{-\infty}^{+\infty} \frac{\rho(\eta)}{\eta(\eta-\xi)} \cdot e^{i\left(\eta-\frac{q_{0}^{2}}{\eta}\right) x} \cdot N_{1}(x, \eta) d \eta \\
\widehat{q}^{*}+\sum_{l=1}^{J} \frac{\xi \cdot b\left(z_{l}\right) e^{i\left(z_{l}-\frac{q_{0}^{2}}{z_{l}}\right) x}}{\left(\xi-z_{l}\right) z_{l} a^{\prime}\left(z_{l}\right)} \cdot N_{2}\left(x, z_{l}\right)+\frac{\xi}{2 \pi i} \int_{-\infty}^{+\infty} \frac{\rho(\eta)}{\eta(\eta-\xi)} \cdot e^{i\left(\eta-\frac{q_{0}^{2}}{\eta}\right) x} \cdot N_{2}(x, \eta) d \eta
\end{array}\right) d \xi .
\end{aligned}
$$

We note that from Eq. (4.46), $q(x)$ is given only in terms of the component $N_{1}$. We can use only the first component of Eq. (5.19) to find this function and hence $q(x)$. Hence to complete the inverse scattering, we reduce the problem to solving an integral equation in terms of the component $N_{1}$ only.

\section{J. Trace formula}

Using $b^{*}\left(-z^{*}\right)=\bar{b}(z)$ and following the analysis in Sec. III, we can get 


$$
\begin{aligned}
& \log a(z)=\log \left(\prod_{j=1}^{J_{1}} \frac{z-z_{j}}{z-\frac{q_{0}^{2}}{z_{j}}} \cdot \frac{z+z_{j}^{*}}{z+\frac{q_{0}^{2}}{z_{j}^{*}}} \cdot \prod_{i=1}^{J_{2}} \frac{z-\widetilde{z}_{i}}{z-\frac{q_{0}^{2}}{\widetilde{z}_{i}}}\right)+\frac{1}{2 \pi i} \int_{-\infty}^{+\infty} \frac{\log \left(1+b(\xi) b^{*}\left(-\xi^{*}\right)\right)}{\xi-z} d \xi, \quad \mathfrak{J} z>0 \\
& \log \bar{a}(z)=\log \left(\prod_{j=1}^{J_{1}} \frac{z-\frac{q_{0}^{2}}{z_{j}}}{z-z_{j}} \cdot \frac{z+\frac{q_{0}^{2}}{z_{j}^{*}}}{z+z_{j}^{*}} \cdot \prod_{i=1}^{J_{2}} \frac{z-\frac{q_{0}^{2}}{\widetilde{z}_{i}}}{z-\widetilde{z}_{i}}\right)-\frac{1}{2 \pi i} \int_{-\infty}^{+\infty} \frac{\log \left(1+b(\xi) b^{*}\left(-\xi^{*}\right)\right)}{\xi-z} d \xi, \quad \mathfrak{J} z<0,
\end{aligned}
$$

where $\mathfrak{R} \widetilde{z}_{i}=0, \mathfrak{R} z_{j} \neq 0$ and $2 J_{1}+J_{2}=J$.

\section{K. Discrete scattering data and their symmetries}

As discussed earlier, the data $a\left(z_{j}\right), \bar{a}\left(\bar{z}_{j}\right)$ are calculated from the trace formulae. The symmetries on the data $b\left(z_{j}\right), \bar{b}\left(\bar{z}_{j}\right), j=1,2, \ldots, J$, can be calculated from their associated eigenfunctions. Using the method in Sec. III L, we can deduce $b\left(z_{j}\right) b^{*}\left(-z_{j}^{*}\right)=-1$. If $\mathfrak{R}_{z_{j}}=0$, we are led to a contradiction. Thus we must have that $\mathfrak{R}_{z_{j}} \neq 0$; consequently, the eigenvalues come in complex conjugate pairs and $J$ must be even.

\section{Reflectioness potentials and soliton solutions}

Reflectioness potentials correspond to zero reflection coefficients, i.e., $\rho(\xi)=0$ and $\bar{\rho}(\xi)=0$ for all real $\xi$. We also note that from the symmetry relation $\bar{b}(z)=-b^{*}\left(-z^{*}\right)$, it follows that the reflection coefficients $\rho(z)=b(z) / a(z), \bar{\rho}(z)=\bar{b}(z) / \bar{a}(z)$ will both vanish when $b(z)=0$ for $z$ on the real axis. By substituting $z=z_{l}$ in (5.19), the system (5.19) reduces to an algebraic equation that determine the functional form of these special potentials. When time dependence is added, the reflectionless potentials correspond to soliton solutions. The reduced equation takes the form

$$
\left(\begin{array}{l}
N_{1}\left(x, z_{l}\right) \\
N_{2}\left(x, z_{l}\right)
\end{array}\right)=\left(\begin{array}{c}
-i \widehat{q} \\
z_{l}
\end{array}\right)+\sum_{j=1}^{J} \frac{z_{l} \cdot \bar{b}\left(\bar{z}_{j}\right) e^{-i\left(\bar{z}_{j}-\frac{q_{0}^{2}}{\bar{z}_{j}}\right) x}}{\left(z_{l}-\bar{z}_{j}\right) \bar{z}_{j} \bar{a}^{\prime}\left(\bar{z}_{j}\right)}\left(\begin{array}{c}
\bar{z}_{j}+\sum_{l=1}^{J} \frac{\bar{z}_{j} \cdot b\left(z_{l}\right) e^{i\left(z_{l}-\frac{q_{0}^{2}}{z_{l}}\right)_{x}}}{\left(\bar{z}_{j}-z_{l}\right) z_{l} a^{\prime}\left(z_{l}\right)} \cdot N_{1}\left(x, z_{l}\right) \\
\widehat{q}^{*}+\sum_{l=1}^{J} \frac{\bar{z}_{j} \cdot b\left(z_{l}\right) e^{i\left(z_{l}-\frac{q_{0}^{2}}{z_{l}}\right) x}}{\left(\bar{z}_{j}-z_{l}\right) z_{l} a^{\prime}\left(z_{l}\right)} \cdot N_{2}\left(x, z_{l}\right)
\end{array}\right) .
$$

The above equations are an algebraic system to solve for $N\left(x, z_{l}\right), l=1,2, \ldots, J$. The potentials are reconstructed from Eq. (4.46) with $\rho(\xi)=0$, i.e.,

$$
q(z)=\widehat{q} \cdot\left[1+\sum_{j=1}^{J} \frac{b\left(z_{j}\right) e^{i\left(z_{j}-\frac{q_{0}^{2}}{z_{j}}\right) x}}{-z_{j}^{2} a^{\prime}\left(z_{j}\right)} \cdot N_{1}\left(x, z_{j}\right)\right] .
$$

Since $a(z) \sim 1$ as $z \rightarrow 0$, by the trace formula when $b(\xi)=0$ in the real axis, we have the constraint $\prod_{j=1}^{J / 2} \frac{\left|z_{j}\right|^{4}}{q_{0}^{4}}=1$.

\section{Reflectionless potential solution: 2-Eigenvalue}

In this subsection, we construct an explicit form for the 2-reflectionless solution (this is the 2 -soliton solution when time dependence is added) by setting $J=2$ and $z_{1}=\xi_{1}+i \eta_{1}$, where $\xi_{1}, \eta_{1}$ $>0$, and $\xi_{1}^{2}+\eta_{1}^{2}=q_{0}^{2}$. Then $a\left(\xi_{1}+i \eta_{1}\right)=a\left(-\xi_{1}+i \eta_{1}\right)=0$ and $\bar{a}\left(\xi_{1}-i \eta_{1}\right)=\bar{a}\left(-\xi_{1}-i \eta_{1}\right)=0$. Thus,

$$
a(z)=\frac{\left(z-\left(\xi_{1}+i \eta_{1}\right)\right)\left(z-\left(-\xi_{1}+i \eta_{1}\right)\right)}{\left(z-\left(\xi_{1}-i \eta_{1}\right)\right)\left(z-\left(-\xi_{1}-i \eta_{1}\right)\right)}, \quad \bar{a}(z)=\frac{\left(z-\left(\xi_{1}-i \eta_{1}\right)\right)\left(z-\left(-\xi_{1}-i \eta_{1}\right)\right)}{\left(z-\left(\xi_{1}+i \eta_{1}\right)\right)\left(z-\left(-\xi_{1}+i \eta_{1}\right)\right)},
$$


and we then find

$$
\begin{aligned}
& a^{\prime}\left(\xi_{1}+i \eta_{1}\right)=\frac{-i \xi_{1}\left(\xi_{1}-i \eta_{1}\right)}{2 q_{0}^{2} \eta_{1}}, \quad a^{\prime}\left(-\xi_{1}+i \eta_{1}\right)=\frac{-i \xi_{1}\left(\xi_{1}+i \eta_{1}\right)}{2 q_{0}^{2} \eta_{1}}, \\
& \bar{a}^{\prime}\left(\xi_{1}-i \eta_{1}\right)=\frac{i \xi_{1}\left(\xi_{1}+i \eta_{1}\right)}{2 q_{0}^{2} \eta_{1}}, \quad \bar{a}^{\prime}\left(-\xi_{1}-i \eta_{1}\right)=\frac{i \xi_{1}\left(\xi_{1}-i \eta_{1}\right)}{2 q_{0}^{2} \eta_{1}} .
\end{aligned}
$$

Moreover, we write $b\left(\xi_{1}+i \eta_{1}\right)=c_{1} e^{i \theta_{1}}$, by $b\left(z_{j}\right) b^{*}\left(-z_{j}^{*}\right)=-1$, we have $b\left(-\xi_{1}+i \eta_{1}\right)=-\frac{1}{c_{1}} e^{i \theta_{1}}$. From $b\left(\frac{q_{0}^{2}}{z}\right)=-\frac{\widehat{q}^{*}}{\widehat{q}} \cdot \bar{b}(z)$, we get

$$
\bar{b}\left(\xi_{1}-i \eta_{1}\right)=-e^{2 i \theta} \cdot b\left(\xi_{1}+i \eta_{1}\right)=-c_{1} e^{i\left(2 \theta+\theta_{1}\right)}, \quad \bar{b}\left(-\xi_{1}-i \eta_{1}\right)=-e^{2 i \theta} \cdot b\left(-\xi_{1}+i \eta_{1}\right)=\frac{1}{c_{1}} e^{i\left(2 \theta+\theta_{1}\right)} .
$$

Hence, we have

$$
\begin{aligned}
& N_{1}\left(x, \xi_{1}+i \eta_{1}\right)=\left(e ^ { 2 \eta _ { 1 } x + i \theta } \cdot \xi _ { 1 } \cdot \left(e^{2 \eta_{1} x+2 i\left(\theta+\theta_{1}\right)} q_{0}\left(\eta_{1}+c_{1}^{2} \eta_{1}-i \xi_{1}\right)-i c_{1}^{2} q_{1} \xi_{1} e^{6 \eta_{1} x}+c_{1} \xi_{1}\left(\xi_{1}+i \eta_{1}\right) e^{3\left(\theta+\theta_{1}\right) i}\right.\right. \\
& \left.\left.+c_{1}\left(\eta_{1}-i \xi_{1}\right)\left(\eta_{1}+c_{1}^{2} \eta_{1}+i c_{1}^{2} \xi_{1}\right) e^{4 \eta_{1} x+i\left(\theta+\theta_{1}\right)}\right)\right) /\left(c_{1}^{2} \xi_{2}^{2} e^{8 \eta_{1} x}+c_{1}^{2} \xi_{1}^{2} e^{4\left(\theta+\theta_{1}\right) i}+e^{4 \eta_{1} x+2 i\left(\theta+\theta_{1}\right)}\right. \\
& \left.\cdot\left(\left(1+c_{1}^{2}\right)^{2} \eta_{1}^{2}+\left(1+c_{1}^{4}\right) \xi_{1}^{2}\right)\right), \\
& \quad N_{1}\left(x,-\xi_{1}+i \eta_{1}\right)=\left(c _ { 1 } \xi _ { 1 } e ^ { 2 \eta _ { 1 } x + i \theta } \cdot \left(e^{4 \eta_{1} x+i\left(\theta+\theta_{1}\right)}\left(\eta_{1}+c_{1}^{2} \eta_{1}-i \xi_{1}\right)\left(\eta_{1}+i \xi_{1}\right)-i c_{1} q_{0} \xi_{1} e^{6 \eta_{1} x}\right.\right. \\
& \left.\left.\quad+c_{1}^{2} \xi_{1}\left(\xi_{1}-i \eta_{1}\right) e^{3 i\left(\theta+\theta_{1}\right)}-c_{1} q_{0}\left(\eta_{1}+c_{1}^{2} \eta_{1}+i c_{1}^{2} \xi_{1}\right) e^{2 \eta_{1} x+2 i\left(\theta+\theta_{1}\right)}\right)\right) /\left(c_{1}^{2} \xi_{1}^{2} e^{8 \eta_{1} x}+c_{1}^{2} \xi_{1}^{2} e^{4 i\left(\theta+\theta_{1}\right)}\right. \\
& \left.\quad+e^{4 \eta_{1} x+2 i\left(\theta+\theta_{1}\right)}\left(\left(1+c_{1}^{2}\right)^{2} \eta_{1}^{2}+\left(1+c_{1}^{4}\right) \xi_{1}^{2}\right)\right) .
\end{aligned}
$$

By (4.51), we can obtain

$$
q(x)=\widehat{q} \cdot\left[1+\frac{c_{1} e^{i \theta_{1}} e^{-2 \eta_{1} x}}{\frac{i \xi_{1}\left(\xi_{1}+i \eta_{1}\right)}{2 \eta_{1}}} N_{1}\left(x, \xi_{1}+i \eta_{1}\right)+\frac{-\frac{1}{c_{1}} e^{i \theta_{1}} e^{-2 \eta_{1} x}}{\frac{i \xi_{1}\left(\xi_{1} i \eta_{1}\right)}{2 \eta_{1}}} N_{1}\left(x,-\xi_{1}+i \eta_{1}\right)\right] .
$$

\section{N. Time evolution}

As in Sec. III O, we find that the scattering data satisfies

$$
\frac{\partial a(t)}{\partial t}=0, \quad \frac{\partial \bar{a}(t)}{\partial t}=0, \quad \frac{\partial b(t)}{\partial t}=-2 i\left(q_{0}^{2}+2 \lambda k\right) b(t) .
$$

Hence, $a(t)$ and $\bar{a}(t)$ are time independent. Moreover,

$$
b\left(\xi_{1}+i \eta_{1}, t\right)=c_{1} e^{i \theta_{1}} \cdot e^{-2 i\left(q_{0}^{2}+2 \xi_{1} \eta_{1} i\right) t}, \quad b\left(-\xi_{1}+i \eta_{1}, t\right)=-\frac{1}{c_{1}} e^{i \theta_{1}} \cdot e^{-2 i\left(q_{0}^{2}-2 \xi_{1} \eta_{1} i\right) t} .
$$

Putting all the above into the formula we had for the reflectionless potential, we obtain the following 2-soliton solution:

$$
\begin{aligned}
& q(x, t)=\left(e ^ { i ( 2 q _ { 0 } ^ { 2 } t + \theta ) } \left(c _ { 1 } ^ { 2 } q _ { 0 } e ^ { 8 t \eta _ { 1 } \sqrt { q _ { 0 } ^ { 2 } - \eta _ { 1 } ^ { 2 } } } \left(-2 \eta_{1}^{2} e^{4 \eta_{1} x+2 i\left(\theta+\theta_{1}\right)}+\left(q_{0}^{2}-\eta_{1}^{2}\right)\left(e^{8 \eta_{1} x}+e^{4 i\left(\theta+\theta_{1}\right)}\right)\right.\right.\right. \\
& +c_{1}^{4} q_{0} e^{4 \eta_{1}\left(x+4 t \sqrt{q_{0}^{2}-\eta_{1}^{2}}\right)+2 i\left(\theta+\theta_{1}\right)} \cdot\left(q_{0}^{2}-2 \eta_{1}^{2}-2 i \eta_{1} \sqrt{q_{0}^{2}-\eta_{1}^{2}}\right)+2 c_{1} \eta_{1} e^{2 \eta_{1} x+4 t \eta_{1} \sqrt{q_{0}^{2}-\eta_{1}^{2}}+i\left(\theta+\theta_{1}\right)} \\
& \cdot\left(-e^{4 \eta_{1} x}+e^{2 i\left(\theta+\theta_{1}\right)}\right)\left(-q_{0}^{2}+\eta_{1}\left(\eta_{1}-i \sqrt{q_{0}^{2}-\eta_{1}^{2}}\right)\right)-2 c_{1}^{3} \eta_{1} e^{2 \eta_{1}\left(x+6 t \sqrt{q_{0}^{2}-\eta_{1}^{2}}\right)+i\left(\theta+\theta_{1}\right)} \cdot\left(-e^{4 \eta_{1} x}+e^{2 i\left(\theta+\theta_{1}\right)}\right) \\
& \left.\left.\cdot\left(-q_{0}^{2}+\eta_{1}\left(\eta_{1}-i \sqrt{q_{0}^{2}-\eta_{1}^{2}}\right)\right)+q_{0} e^{4 \eta_{1} x+2 i\left(\theta+\theta_{1}\right)} \cdot\left(q_{0}^{2}+2 i \eta_{1}\left(i \eta_{1}+\sqrt{q_{0}^{2}-\eta_{1}^{2}}\right)\right)\right)\right) / \\
& \left(q_{0}^{2} e^{4 \eta_{1} x+2 i\left(\theta+\theta_{1}\right)}+c_{1}^{4} q_{0}^{2} e^{4 \eta_{1}\left(x+4 t \sqrt{q_{0}^{2}-\eta_{1}^{2}}\right)+2 i\left(\theta+\theta_{1}\right)}-c_{1}^{2} e^{8 t \eta_{1} \sqrt{q_{0}^{2}-\eta_{1}^{2}}}\left(-2 \eta_{1}^{2} e^{4 \eta_{1} x+2 i\left(\theta+\theta_{1}\right)}\right.\right. \\
& \left.\left.+\left(\eta_{1}^{2}-q_{0}^{2}\right)\left(e^{8 \eta_{1} x+4 i\left(\theta+\theta_{1}\right)}\right)\right)\right) .
\end{aligned}
$$




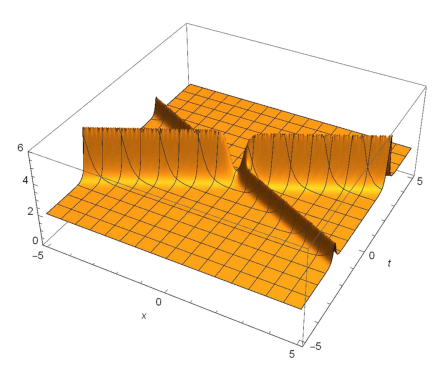

(a)

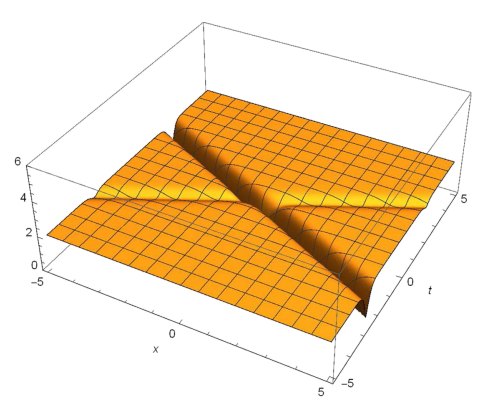

(b)

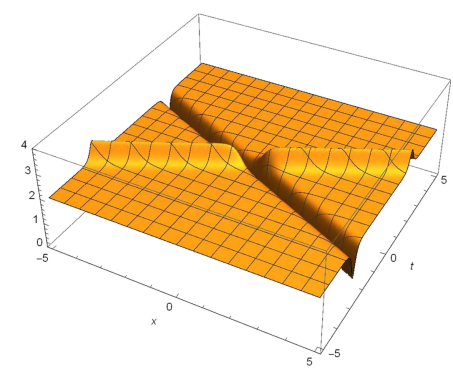

(c)

FIG. 8. (a) The amplitude of $q(x, t)$ with $\theta=0, \theta_{1}=-\frac{3 \pi}{4}, c_{1}=\sqrt{2}, \eta_{1}=\sqrt{3}$, and $q_{0}=2$. (b) The amplitude of $q(x, t)$ with $\theta$ $=0, \theta_{1}=\frac{3 \pi}{4}, c_{1}=\sqrt{2}, \eta_{1}=\sqrt{3}$, and $q_{0}=2$. (c) The amplitude of $q(x, t)$ with $\theta=0, \theta_{1}=\pi, c_{1}=\sqrt{2}, \eta_{1}=\sqrt{3}$, and $q_{0}=2$.

Typical 2-soliton solutions, which travel and interact, including the one in Ref. 15, are shown in Fig. 8. In terms of magnitude, Fig. 8(a) shows the interaction of two waves of elevation, Fig. 8(b) shows the interaction of two depressive waves (i.e., two "dips"), and Fig. 8(c) illustrates the interaction of one elevated wave and one depressive wave.

If we choose $q_{0}=2, \eta_{1}=\sqrt{3}, \theta=0, c_{1}=\sqrt{\frac{p_{2}-p_{1}}{p_{2}+p_{1}}}$, and $e^{2 i \theta}=\frac{-p_{3}-i p_{4}}{p_{3}-i p_{4}}$, we can get the general formula in Ref. 15.

\section{THE CASE OF $\sigma=1$ WITH $\theta_{+}-\theta_{-}=\pi$}

The final case we will study is the nonlocal nonlinear Schrödinger (NLS) equation (1.1) with $\sigma$ $=1$

$$
i q_{t}(x, t)=q_{x x}(x, t)-2 q^{2}(x, t) q^{*}(-x, t)
$$

with nonzero boundary conditions (NZBCs)

$$
q(x, t) \rightarrow q_{ \pm}(t)=q_{0} e^{-2 i q_{0}^{2} t+i \theta_{ \pm}} \text {, as } x \rightarrow \pm \infty,
$$

where $q_{0}>0,0 \leq \theta_{ \pm}<2 \pi, \theta_{+}-\theta_{-}=\pi$.

The analysis of this case is similar to the NZBCs studied in Sec. IV. As discussed in Sec. IV, it is natural to introduce the eigenfunctions defined by the following boundary conditions:

$$
\begin{aligned}
& \phi(x, k) \sim w e^{-i \lambda x}, \quad \bar{\phi}(x, k) \sim \bar{w} e^{i \lambda x} \text { as } x \rightarrow-\infty, \\
& \psi(x, k) \sim v e^{i \lambda x}, \quad \bar{\psi}(x, k) \sim \bar{v} e^{-i \lambda x} \text { as } x \rightarrow+\infty,
\end{aligned}
$$

where

$$
w=\left(\begin{array}{c}
\lambda+k \\
i q_{+}^{*}
\end{array}\right), \quad \bar{w}=\left(\begin{array}{c}
-i q_{-} \\
\lambda+k
\end{array}\right), \quad v=\left(\begin{array}{c}
-i q_{+} \\
\lambda+k
\end{array}\right), \quad \bar{v}=\left(\begin{array}{c}
\lambda+k \\
i q_{-}^{*}
\end{array}\right)
$$

satisfy the boundary conditions.

As in Sec. III, we consider functions with constant boundary conditions and define the same bounded eigenfunctions $M(x, k), N(x, k), \bar{M}(x, k), \bar{N}(x, k)$ except we use the boundary conditions defined in (6.5).

The bounded eigenfunctions can be represented by means of integral equations, the formulae are the same as in (3.9)-(3.12) except for the constant terms, which are defined above in (6.5).

\section{A. Symmetry reductions}

Taking into account boundary conditions, we can obtain (5.7). The scattering relations are the same as in Eqs. (3.41)-(3.45) except for the relation between $b$ and $\bar{b}$, i.e., $b^{*}\left(-k^{*}\right)=\bar{b}(k)$. 
Similarly, we can define the eigenfunctions and scattering data on the left/right edge of the cut for $k \in\left(-i q_{0}, i q_{0}\right)$. Then

$$
\phi^{\mp}(x, k)=\frac{\lambda^{\mp}+k}{-i q_{-}} \cdot \bar{\phi}^{ \pm}(x, k), \quad \psi^{\mp}(x, k)=\frac{\lambda^{\mp}+k}{\widetilde{i q}_{-}^{*}} \cdot \bar{\psi}^{ \pm}(x, k)
$$

for $k \in\left(-i q_{0}, i q_{0}\right)$. Moreover, we have

$$
a^{ \pm}(k)=-\bar{a}^{\mp}(k), \quad b^{ \pm}(k)=\frac{q_{0}^{2}}{q_{+} \cdot q_{-}} \cdot \bar{b}^{\mp}(k) \text { for } k \in\left(-i q_{0}, i q_{0}\right) .
$$

\section{B. Uniformization coordinates}

Before discussing the properties of scattering data and solving the inverse problem, we introduce a uniformization variable $z$, defined by the conformal mapping: $z=z(k)=k+\lambda(k)$, and the inverse mapping is given by $k=k(z)=\frac{1}{2}\left(z-\frac{q_{0}^{2}}{z}\right)$. Then $\lambda(z)=\frac{1}{2}\left(z+\frac{q_{0}^{2}}{z}\right)$.

\section{Symmetries via uniformization coordinates}

It is known that (1) when $z \rightarrow-z^{*}$, then $(k, \lambda) \rightarrow\left(-k^{*},-\lambda^{*}\right)$ and (2) when $z \rightarrow-\frac{q_{0}^{2}}{z}$, then $(k, \lambda) \rightarrow$ $(k,-\lambda)$. Hence, we can deduce the same formulae as in (5.10) and (5.12) although $z$ has a different representation here. Moreover,

$$
\begin{gathered}
\phi\left(x,-\frac{q_{0}^{2}}{z}\right)=\frac{\frac{q_{0}^{2}}{z}}{i q_{-}} \bar{\phi}(x, z), \quad \psi\left(x,-\frac{q_{0}^{2}}{z}\right)=\frac{-i q_{+}}{z} \bar{\psi}(x, z), \quad z \in D^{-}, \\
a\left(-\frac{q_{0}^{2}}{z}\right)=-\bar{a}(z), \quad z \in D^{-}, \quad b\left(-\frac{q_{0}^{2}}{z}\right)=\frac{q_{0}^{2}}{q_{+} \cdot q_{-}} \cdot \bar{b}(z),
\end{gathered}
$$

where $D^{-}$represents the white regions of Fig. 5 .

\section{Asymptotic behavior of eigenfunctions and scattering data}

In order to solve the inverse problem, one has to determine the asymptotic behavior of eigenfunctions and scattering data both as $z \rightarrow \infty$ in $\mathbb{K}_{1}$ and as $z \rightarrow 0$ in $\mathbb{K}_{2}$. We have

$$
\begin{gathered}
N(x, z) \sim\left(\begin{array}{c}
-i q(x) \\
z
\end{array}\right), \quad z \rightarrow \infty, \quad N(x, z) \sim\left(\begin{array}{c}
-i q_{+} \\
z \cdot \frac{q^{*}(-x)}{q_{-}^{*}}
\end{array}\right), \quad z \rightarrow 0, \\
a(z)=\left\{\begin{array}{ll}
1, \quad z \rightarrow \infty, \\
-1, \quad z \rightarrow 0,
\end{array} \quad \bar{a}(z)= \begin{cases}1, & z \rightarrow \infty, \\
-1, & z \rightarrow 0,\end{cases} \right. \\
\lim _{z \rightarrow \infty} z b(z)=0, \quad \lim _{z \rightarrow 0} \frac{b(z)}{z^{2}}=0 .
\end{gathered}
$$

\section{E. Left and right scattering problems}

Using the methods in the second case, we can get

$$
\begin{gathered}
\bar{N}(x, z)=\left(\begin{array}{c}
z \\
i q_{-}^{*}
\end{array}\right)+\sum_{j=1}^{J} \frac{z \cdot b\left(z_{j}\right) e^{i\left(z_{j}+\frac{q_{0}^{2}}{z_{j}}\right) x} \cdot N\left(x, z_{j}\right)}{\left(z-z_{j}\right) z_{j} a^{\prime}\left(z_{j}\right)}+\frac{z}{2 \pi i} \int_{\Sigma} \frac{\rho(\xi)}{\xi(\xi-z)} \cdot e^{i\left(\xi+\frac{q_{0}^{2}}{\xi}\right) x} \cdot N(x, \xi) d \xi, \\
N(x, z)=\left(\begin{array}{c}
-i q_{+} \\
z
\end{array}\right)+\sum_{j=1}^{\bar{J}} \frac{z \cdot \bar{b}\left(\bar{z}_{j}\right) e^{-i\left(\bar{z}_{j}+\frac{q_{0}^{2}}{\bar{z}_{j}}\right) x} \cdot \bar{N}\left(x, \bar{z}_{j}\right)}{\left(z-\bar{z}_{j}\right) \bar{z}_{j} \bar{a}^{\prime}\left(\bar{z}_{j}\right)}-\frac{z}{2 \pi i} \int_{\Sigma} \frac{\bar{\rho}(\xi)}{\xi(\xi-z)} \cdot e^{-i\left(\xi+\frac{q_{0}^{2}}{\xi}\right) x} \cdot \bar{N}(x, \xi) d \xi,
\end{gathered}
$$




$$
\begin{gathered}
\bar{M}(x, z)=\left(\begin{array}{c}
-i q_{-} \\
z
\end{array}\right)+\sum_{j=1}^{J} \frac{-z \cdot \bar{b}\left(z_{j}\right) M\left(x, z_{j}\right) e^{-i\left(z_{j}+\frac{q_{0}^{2}}{z_{j}}\right) x}}{\left(z-z_{j}\right) z_{j} a^{\prime}\left(z_{j}\right)}+\frac{z}{2 \pi i} \int_{\Sigma} \frac{\rho^{*}\left(-\xi^{*}\right)}{\xi(\xi-z)} \cdot e^{-i\left(\xi+\frac{q_{0}^{2}}{\xi}\right) x} \cdot M(x, \xi) d \xi, \\
M(x, z)=\left(\begin{array}{c}
z \\
i q_{+}^{*}
\end{array}\right)+\sum_{j=1}^{\bar{J}} \frac{-z \cdot b\left(\bar{z}_{j}\right) \bar{M}\left(x, \bar{z}_{j}\right) e^{i\left(\bar{z}_{j}+\frac{q_{0}^{2}}{\bar{z}_{j}}\right) x}}{\left(z-\bar{z}_{j}\right) \bar{z}_{j} \bar{a}^{\prime}\left(\bar{z}_{j}\right)}-\frac{z}{2 \pi i} \int_{\Sigma} \frac{\bar{\rho}^{*}\left(-\xi^{*}\right)}{\xi(\xi-z)} \cdot e^{i\left(\xi+\frac{q_{0}^{2}}{\xi}\right) x} \cdot \bar{M}(x, \xi) d \xi,
\end{gathered}
$$

where we recall from the second case $\Sigma:=\left(-\infty,-q_{0}\right) \cup\left(q_{0},+\infty\right) \cup \overrightarrow{\left(q_{0},-q_{0}\right)} \cup\left\{q_{0} e^{i \theta}, \pi \leq \theta \leq\right.$ $2 \pi\}_{\text {clockwise, upper circle }} \cup\left\{q_{0} e^{i \theta},-\pi \leq \theta \leq 0\right\}_{\text {anticlockwise, lower circle. }}$.

\section{F. Recovery of the potentials}

Note that $N_{1}(x, z) \sim-i q(x)$ as $z \rightarrow \infty$ and

$$
N_{1}(x, z) \sim-i q_{+}+\sum_{j=1}^{\bar{J}} \frac{\bar{b}\left(\bar{z}_{j}\right) e^{-i\left(\bar{z}_{j}+\frac{q_{0}^{2}}{\bar{z}_{j}}\right) x} \cdot \bar{N}_{1}\left(x, \bar{z}_{j}\right)}{\bar{z}_{j} \bar{a}^{\prime}\left(\bar{z}_{j}\right)}+\frac{1}{2 \pi i} \int_{\Sigma} \frac{\bar{\rho}(\xi)}{\xi} \cdot e^{-i\left(\xi+\frac{q_{0}^{2}}{\xi}\right) x} \cdot \bar{N}_{1}(x, \xi) d \xi,
$$

we have

$$
q(x)=q_{+}+i \sum_{j=1}^{\bar{J}} \frac{\bar{b}\left(\bar{z}_{j}\right) e^{-i\left(\bar{z}_{j}+\frac{q_{0}^{2}}{\bar{z}_{j}}\right) x} \cdot \bar{N}_{1}\left(x, \bar{z}_{j}\right)}{\bar{z}_{j} \bar{a}^{\prime}\left(\bar{z}_{j}\right)}+\frac{1}{2 \pi} \int_{\Sigma} \frac{\bar{\rho}(\xi)}{\xi} \cdot e^{-i\left(\xi+\frac{q_{0}^{2}}{\xi}\right) x} \cdot \bar{N}_{1}(x, \xi) d \xi .
$$

\section{G. Trace formula}

As in Sec. V K, we can show that $b\left(z_{j}\right) b^{*}\left(-z_{j}^{*}\right)=-1$, which implies that $\mathfrak{R}_{z_{j}} \neq 0$ and $J$ is even. Hence, we can get the trace formula as follows:

$$
\begin{aligned}
& \log a(z)=\log \left(\prod_{j=1}^{J / 2} \frac{z-z_{j}}{z+\frac{q_{0}^{2}}{z_{j}}} \cdot \frac{z+z_{j}^{*}}{z-\frac{q_{0}^{2}}{z_{j}^{*}}}\right)+\frac{1}{2 \pi i} \int_{\Sigma} \frac{\log \left(1+b(\xi) b^{*}\left(-\xi^{*}\right)\right)}{\xi-z} d \xi, \quad z \in D^{+}, \\
& \log \bar{a}(z)=\log \left(\prod_{j=1}^{J / 2} \frac{z+\frac{q_{0}^{2}}{z_{j}}}{z-z_{j}} \cdot \frac{z-\frac{q_{0}^{2}}{z_{j}^{*}}}{z+z_{j}^{*}}\right)-\frac{1}{2 \pi i} \int_{\Sigma} \frac{\log \left(1+b(\xi) b^{*}\left(-\xi^{*}\right)\right)}{\xi-z} d \xi, \quad z \in D^{-},
\end{aligned}
$$

where $\mathfrak{R}_{z_{j}} \neq 0$. We claim that this case does not admit soliton solutions. Indeed, by the asymptotic of $a(z)$, i.e., $a(z) \sim-1$ as $z \rightarrow 0$ and the fact $\mathfrak{R}_{z_{j}} \neq 0$. Moreover, the trace formula yields

$$
a(z)=\prod_{j=1}^{J / 2} \frac{z-z_{j}}{z+\frac{q_{0}^{2}}{z_{j}}} \cdot \frac{z+z_{j}^{*}}{z-\frac{q_{0}^{2}}{z_{j}^{*}}} \exp \left(\frac{1}{2 \pi i} \int_{\Sigma} \frac{\log \left(1+b(\xi) b^{*}\left(-\xi^{*}\right)\right)}{\xi-z} d \xi\right) .
$$

Since the exponential term is real for all $\xi \in \Sigma$, it implies that

$$
\prod_{j=1}^{J / 2} \frac{\left|z_{j}\right|^{4}}{q_{0}^{4}} \exp \left(\frac{1}{2 \pi i} \int_{\Sigma} \frac{\log \left(1+b(\xi) b^{*}\left(-\xi^{*}\right)\right)}{\xi} d \xi\right)=-1,
$$

which is a contradiction. Thus, in this case there are no solitons. 


\section{H. Closing the system}

This time the equation will not have a discrete spectrum contribution; to close the system, by the symmetry relations between the eigenfunctions, we have

$$
\begin{aligned}
\left(\begin{array}{c}
\bar{N}_{1}(x, z) \\
\bar{N}_{2}(x, z)
\end{array}\right)= & \left(\begin{array}{c}
z \\
i q_{-}^{*}
\end{array}\right)+\frac{z}{2 \pi i} \int_{\Sigma} \frac{\rho(\xi)}{\xi(\xi-z)} \cdot e^{i\left(\xi+\frac{q_{0}^{2}}{\xi}\right) x} . \\
& \left(\begin{array}{c}
-i q_{+}-\frac{\xi}{2 \pi i} \int_{\Sigma} \frac{\bar{\rho}(\eta)}{\eta(\eta-\xi)} \cdot e^{-i\left(\eta+\frac{q_{0}^{2}}{\eta}\right) x} \cdot \bar{N}_{1}(x, \eta) d \eta \\
\xi-\frac{\xi}{2 \pi i} \int_{\Sigma} \frac{\bar{\rho}(\eta)}{\eta(\eta-\xi)} \cdot e^{-i\left(\eta+\frac{q_{0}^{2}}{\eta}\right) x} \cdot \bar{N}_{2}(x, \eta) d \eta
\end{array}\right) d \xi
\end{aligned}
$$

\section{Time evolution}

Similar to the case in Sec. IV, we can get

$$
\frac{\partial a(t)}{\partial t}=0, \quad \frac{\partial \bar{a}(t)}{\partial t}=0, \quad \frac{\partial b(t)}{\partial t}=2 i\left(q_{0}^{2}-2 \lambda k\right) b(t), \quad \frac{\partial \bar{b}(t)}{\partial t}=-2 i\left(q_{0}^{2}-2 \lambda k\right) \bar{b}(t) .
$$

Then

$$
\rho(z, t)=\rho(z, 0) e^{2 i\left[q_{0}^{2}-\frac{1}{2}\left(\xi^{2}-\frac{q_{0}^{4}}{\xi^{2}}\right)\right] t}, \quad \bar{\rho}(z, t)=\bar{\rho}(z, 0) e^{-2 i\left[q_{0}^{2}-\frac{1}{2}\left(\xi^{2}-\frac{q_{0}^{4}}{\xi^{2}}\right)\right] t} .
$$

It yields

$$
q(x, t)=q_{0} e^{-2 i q_{0}^{2} t+i \theta_{+}}+\frac{1}{2 \pi} \int_{\Sigma} \frac{\bar{\rho}(\xi, 0) e^{-2 i\left[q_{0}^{2}-\frac{1}{2}\left(\xi^{2}-\frac{q_{0}^{4}}{\xi^{2}}\right)\right] t}}{\xi} e^{-i\left(\xi+\frac{q_{0}^{2}}{\xi}\right) x} \cdot \bar{N}_{1}(x, \xi, t) d \xi .
$$

In principle, we can formulate integral equations for $\bar{M}_{2}$ via the symmetry between $\bar{M}$ and $\bar{N}$ to obtain $q(x)$ as done in Sec. IV.

\section{MODULATIONAL INSTABILITY}

In this section, we study the modulation instability of a constant (in space) amplitude solution to the nonlocal NLS equation (1.1). More precisely, we study linear stability properties of the family of solutions defined by

$$
q(x, t)=A e^{i \omega t},
$$

where $A$ is a constant complex amplitude and $\omega$ is a real frequency. In the limit $x \rightarrow+\infty$, we define $A \equiv A_{+}$, and when $x \rightarrow-\infty$, we let $A \equiv A_{-}$. If we let $A_{ \pm}=q_{0} e^{i \theta_{ \pm}}$, then in order for Eq. (7.1) to be a solution to (1.1) we require that $\theta_{+}-\theta_{-}=0$ or $\pi$. Thus, we distinguish here between four different cases: $\theta_{+}-\theta_{-}=0, \sigma= \pm 1$ and $\theta_{+}-\theta_{-}=\pi, \sigma= \pm 1$.

\section{A. Modulational instability I: $\theta_{+}-\theta_{-}=0$}

In this case, we have $A_{+}=A_{-} \equiv A$ and $\omega=2 \sigma|A|^{2}$. To study modulational instability, we write solutions to (1.1) in the form

$$
q(x, t)=(A+\varepsilon \tilde{q}(x, t)) e^{2 i \sigma|A|^{2} t} .
$$

Substituting (7.2) into (1.1) and retaining terms up to order $\varepsilon$, we obtain

$$
i \tilde{q}_{t}(x, t)=\tilde{q}_{x x}(x, t)-2 \sigma|A|^{2} \tilde{q}(x, t)-2 \sigma A^{2} \tilde{q}^{*}(-x, t) .
$$

Equation (7.3) governs the dynamical evolution of the perturbation $\tilde{q}(x, t)$ subject to the boundary conditions; $\tilde{q}(x, t)$ decays very fast at plus and minus infinity. We next decompose the perturbation in terms of its Fourier integral representation

$$
\tilde{q}(x, t)=\int_{-\infty}^{+\infty}\left(\tilde{q}_{1}(k) e^{i \lambda t}+\tilde{q}_{2}(k) e^{-i \lambda^{*} t}\right) e^{i k x} d k .
$$


It is evident that the constant solution is modulationally unstable if the eigenvalue $\lambda$ is complex. After some algebra, we find

$$
\lambda^{2}=k^{2}\left(k^{2}+4 \sigma|A|^{2}\right) .
$$

Thus we conclude that when $\sigma=+1$, the solution (7.1) is modulationaly stable, whereas it is modulationaly unstable for $\sigma=-1$.

The unstable case corresponds to having two eigenvalues on the imaginary axis or two solitons that are stationary and oscillate in time, as discussed in case 4.

\section{B. Modulational instability II: $\theta_{+}-\theta_{-}=\pi$}

In this second case, we find $A_{+}=-A_{-} \equiv A$ thus $\omega=-2 \sigma|A|^{2}$. Under this situation, the linear stability analysis proceeds as before by writing a perturbative solution to (1.1) in the form (7.2) with the exception that $q^{*}(-x, t)=\left(-A^{*}+\varepsilon \tilde{q}^{*}(-x, t)\right) e^{-2 i \sigma|A|^{2} t}$. Substituting (7.2) into (1.1) and retaining terms up to order $\varepsilon$, we now obtain

$$
i \tilde{q}_{t}(x, t)=\tilde{q}_{x x}(x, t)+2 \sigma|A|^{2} \tilde{q}(x, t)-2 \sigma A^{2} \tilde{q}^{*}(-x, t) .
$$

Decomposing the perturbation in terms of its Fourier integral representation (7.4), we then find

$$
\lambda^{2}=\left(k^{2}-2 \sigma|A|^{2}\right)^{2}+4|A|^{2} .
$$

It is thus evident that the constant amplitude solution is modulationally stable for both signs of $\sigma$.

\section{CONCLUSION}

In this paper, the nonlocal NLS equation (1.1) with nonzero boundary values at infinity is considered. Depending on the signs of nonlinearity $\sigma= \pm 1$ and phase difference of the boundary values from plus infinity to minus infinity $\Delta \theta=\theta_{+}-\theta_{-}$, there are four distinct cases to consider when the amplitude at infinity is constant.

The direct scattering problem and corresponding analytic properties and symmetries of the eigenfunctions and scattering data are obtained. The inverse problem is constructed via a RiemannHilbert problem formulated from both the right and left in terms of a convenient uniformization variable. The simplest pure soliton solutions are obtained. When $\sigma=-1, \Delta \theta=\pi$, a pure one-soliton solution is found; when $\sigma=-1, \Delta \theta=0$, a pure stationary two-soliton solution is obtained; and with $\sigma=1, \Delta \theta=0$ a traveling bidirectional interacting two-soliton solution is found. In the final case $\sigma$ $=1, \Delta \theta=\pi$, there are no soliton solutions allowed. A number of box potentials are analyzed; their associated eigenvalues are found and in all cases shown to be consistent with the prior analytical results. The modulational instability of a plane wave is also discussed.

\section{ACKNOWLEDGMENTS}

The authors are pleased to acknowledge Justin T. Cole and Yi-Ping Ma for considerable assistance in the figures of this paper and in Mathematica, respectively. M.J.A. was partially supported by NSF under Grant No. DMS-1712793.

\section{APPENDIX: DETAILED ANALYSIS FOR SECTION III}

\section{Proof of Theorem 3.2}

For $k \in\left(-\infty,-q_{0}\right) \cup\left(q_{0},+\infty\right)$, the matrices $P_{-i \lambda}(k)^{ \pm}$and $P_{i \lambda}^{ \pm}$are defined as follows:

$$
P_{-i \lambda}^{ \pm}(k)=\frac{1}{2 \lambda}\left(\begin{array}{cc}
\lambda+k & i q_{ \pm} \\
-i q_{\mp}^{*} & \lambda-k
\end{array}\right), \quad P_{i \lambda}^{ \pm}(k)=\frac{1}{2 \lambda}\left(\begin{array}{cc}
\lambda-k & -i q_{ \pm} \\
i q_{\mp}^{*} & \lambda+k
\end{array}\right) .
$$


We have $\left(P_{i \lambda}^{ \pm}\right)^{2}=P_{i \lambda}^{ \pm},\left(P_{-i \lambda}^{ \pm}\right)^{2}=P_{-i \lambda}^{ \pm}, P_{i \lambda}^{ \pm}+P_{-i \lambda}^{ \pm}=I_{2}$, and $P_{i \lambda}^{ \pm} P_{-i \lambda}^{ \pm}=P_{-i \lambda}^{ \pm} P_{i \lambda}^{ \pm}=0$. Moreover,

$$
\left(i k J+Q_{ \pm}\right) P_{-i \lambda}^{ \pm}(k)=-i \lambda P_{-i \lambda}^{ \pm}(k)
$$

and

$$
\left(i k J+Q_{ \pm}\right) P_{i \lambda}^{ \pm}(k)=i \lambda P_{i \lambda}^{ \pm}(k) .
$$

Then, we can rewrite the Green's functions in terms of the projectors to find

$$
\begin{aligned}
& M(x, k)=\left(\begin{array}{c}
\lambda+k \\
-i q_{+}^{*}
\end{array}\right)+\int_{-\infty}^{x}\left[P_{-i \lambda}^{-}+e^{2 i \lambda\left(x-x^{\prime}\right)} P_{i \lambda}^{-}\right]\left(\left(Q-Q_{-}\right) M\right)\left(x^{\prime}, k\right) d x^{\prime}, \\
& \bar{M}(x, k)=\left(\begin{array}{c}
-i q_{-} \\
\lambda+k
\end{array}\right)+\int_{-\infty}^{x}\left[e^{2 i \lambda\left(x^{\prime}-x\right)} P_{-i \lambda}^{-}+P_{i \lambda}^{-}\right]\left(\left(Q-Q_{-}\right) M\right)\left(x^{\prime}, k\right) d x^{\prime}, \\
& N(x, k)=\left(\begin{array}{c}
-i q_{+} \\
\lambda+k
\end{array}\right)+\int_{x}^{+\infty}\left[e^{2 i \lambda\left(x^{\prime}-x\right)} P_{-i \lambda}^{+}+P_{i \lambda}^{+}\right]\left(\left(Q-Q_{+}\right) M\right)\left(x^{\prime}, k\right) d x^{\prime}, \\
& \bar{N}(x, k)=\left(\begin{array}{c}
\lambda+k \\
-i q_{-}^{*}
\end{array}\right)+\int_{x}^{+\infty}\left[P_{-i \lambda}^{+}+e^{2 i \lambda\left(x-x^{\prime}\right)} P_{i \lambda}^{+}\right]\left(\left(Q-Q_{+}\right) M\right)\left(x^{\prime}, k\right) d x^{\prime} .
\end{aligned}
$$

The projections $P_{-i \lambda}^{ \pm}(k)$ and $P_{i \lambda}^{ \pm}(k)$ admit a natural continuation to $k \in \mathbb{K} \backslash\left\{ \pm q_{0}\right\}$, i.e., $\lambda \in \mathbb{C} \backslash\{0\}$. Taking into account that these projections are singular matrices, we can show that their $l^{2}$-norm is given by

$$
\left\|P_{-i \lambda}^{ \pm}(k)\right\|_{2}=\left\|P_{i \lambda}^{ \pm}(k)\right\|_{2}=\sqrt{\frac{|\lambda-k|^{2}+|\lambda+k|^{2}+2 q_{0}^{2}}{4|\lambda|^{2}}}=\frac{\sqrt{2|\lambda|^{2}+2|k|^{2}+2 q_{0}^{2}}}{2|\lambda|} .
$$

In particular, if $k \in\left(-\infty,-q_{0}\right] \cup\left[q_{0},+\infty\right)$, then

$$
\left\|P_{-i \lambda}^{ \pm}(k)\right\|_{2}=\frac{|k|}{|\lambda|}
$$

We consider the Neumann series

$$
M(x, k)=\sum_{n=0}^{\infty} M^{(n)}(x, k)
$$

where

$$
\begin{gathered}
M^{(0)}(x, k)=\left(\begin{array}{c}
\lambda+k \\
-i q_{0} e^{-2 i q_{0}^{2} t-i \theta_{+}}
\end{array}\right), \\
M^{(n+1)}(x, k)=\int_{-\infty}^{x}\left[P_{-i \lambda}^{-}+e^{2 i \lambda\left(x-x^{\prime}\right)} P_{i \lambda}^{-}\right]\left(\left(Q-Q_{-}\right) M^{(n)}\right)\left(x^{\prime}, k\right) d x^{\prime} .
\end{gathered}
$$

Then

$$
\left\|M^{(n+1)}(x, k)\right\| \leq \sqrt{|\lambda+k|^{2}+q_{0}^{2}}+\frac{2|k|}{|\lambda|} \int_{-\infty}^{x}\left\|Q\left(x^{\prime}\right)-Q_{-}\right\| \cdot\left\|M^{(n)}\left(x^{\prime}, k\right)\right\| d x^{\prime}
$$

if $x^{\prime} \leq x$ and $\lambda \in U_{+} \cup(-\infty, 0) \cup(0,+\infty)$.

Since the entries of matrix $Q-Q_{-}$belong to $L^{1}(\mathbb{R})$, using the identities

$$
\begin{aligned}
& \frac{1}{j !} \int_{-\infty}^{x}|f(\xi)|\left[\int_{-\infty}^{\xi}\left|f\left(\xi^{\prime}\right)\right| d \xi^{\prime}\right]^{j} d \xi \\
& =\frac{1}{(j+1) !} \int_{-\infty}^{x} \frac{d}{d \xi}\left[\int_{-\infty}^{\xi}\left|f\left(\xi^{\prime}\right)\right| d \xi^{\prime}\right]^{j+1} d \xi \\
& =\frac{1}{(j+1) !}\left[\int_{-\infty}^{x}|f(\xi)| d \xi\right]^{j+1},
\end{aligned}
$$

we can get the Neumann series which is uniformly convergent for $k \in \overline{\mathbb{C}}_{1} \backslash\left\{ \pm q_{0}\right\}$. It follows that $M(x$, $k$ ) is analytic for $k \in \mathbb{C}_{1}$ because a uniformly convergent series of analytic functions converges to an analytic function. Similarly, $M(x, k)$ is continuous for $k \in \overline{\mathbb{C}}_{1} \backslash\left\{ \pm q_{0}\right\}$. 
To extend the continuity properties at $k= \pm q_{0}$, we rewrite $P_{-i \lambda}^{-}+e^{2 i \lambda\left(x-x^{\prime}\right)} P_{i \lambda}^{-}$as $I_{2}+\left[e^{2 i \lambda\left(x-x^{\prime}\right)}-1\right] P_{i \lambda}^{-}$ and use the estimation

$$
\begin{aligned}
\left\|I_{2}+\left[e^{2 i \lambda\left(x-x^{\prime}\right)}-1\right] P_{i \lambda}^{-}\right\| \leq 1+2\left|x-x^{\prime}\right| \cdot|k| & \leq \max \{1,2|k|\}(1+|x|)\left(1+\left|x^{\prime}\right|\right) \\
& \leq \max \{1,2|k|\}(1+|x|)^{2}
\end{aligned}
$$

and the fact that $f \in L^{1,2}(\mathbb{R}) \subseteq L^{1}(\mathbb{R})$, the proof then proceeds as before under the condition that the entries of $Q-Q_{-}$belong to $L^{1,2}(\mathbb{R})$. Similarly, the proof for $N(x, k), \bar{M}(x, k)$, and $\bar{N}(x, k)$ can be done by using the above method.

\section{Proof of Theorem 3.4}

The integral equation of $M(x, k)$ can be written in the alternative form

$$
M_{1}(x, k)=\lambda+k+\int_{-\infty}^{x} \int_{-\infty}^{y}\left(q(y)-q_{-}\right)\left(-q^{*}(-z)+q_{+}^{*}\right) e^{2 i \lambda(y-z)} M_{1}(z, k) d z d y
$$

and

$$
M_{2}(x, k)=-i q_{+}^{*}+\int_{-\infty}^{x} e^{2 i \lambda(x-y)}\left(-q^{*}(-y)+q_{+}^{*}\right) M_{1}(y, k) d y,
$$

where $M(x, k)=\left(\begin{array}{l}M_{1}(x, k) \\ M_{2}(x, k)\end{array}\right)$.

Since the entries of $Q-Q_{ \pm}$do not grow faster than $e^{-a x^{2}}$, it is easy to see that $M(x, k)$ is analytic in the Riemann surface $\mathbb{K}$. Similarly, we can prove $N(x, k), \bar{M}(x, k)$, and $\bar{N}(x, k)$ are also analytic in $\mathbb{K}$.

\section{Asymptotic behavior of eigenfunctions for the case of $\sigma=-1$ with $\theta_{+}-\theta_{-}=\pi$}

$$
\begin{aligned}
& M(x, z) \sim\left\{\begin{array}{cc}
\left(\begin{array}{c}
z \\
-i q^{*}(-x)
\end{array}\right), & z \rightarrow \infty, \\
\left(\begin{array}{c}
-z \cdot \frac{q(x)}{q_{+}} \\
i q_{-}^{*}
\end{array}\right), \quad z \rightarrow 0,
\end{array}\right. \\
& N(x, z) \sim \begin{cases}\left(\begin{array}{c}
-i q(x) \\
z
\end{array}\right), & z \rightarrow \infty, \\
\left(\begin{array}{c}
-i q_{+} \\
z \cdot \frac{q^{*}(-x)}{q_{-}^{*}}
\end{array}\right), & z \rightarrow 0,\end{cases} \\
& \bar{M}(x, z) \sim\left\{\begin{array}{l}
\left(\begin{array}{c}
-i q(x) \\
z
\end{array}\right), \quad z \rightarrow \infty, \\
\left(\begin{array}{c}
i q_{+} \\
-z \cdot \frac{q^{*}(-x)}{q_{-}^{*}}
\end{array}\right), \quad z \rightarrow 0 .
\end{array}\right.
\end{aligned}
$$

\section{Time evolution for the case of $\sigma=-1$ with $\theta_{+}-\theta_{-}=\pi$}

Since

$$
v_{t}=\left(\begin{array}{cc}
2 i k^{2}-i q(x, t) q^{*}(-x, t) & -2 k q(x, t)-i q_{x}(x, t) \\
2 k q^{*}(-x, t)+i q_{x}^{*}(-x, t) & -2 i k^{2}+i q(x, t) q^{*}(-x, t)
\end{array}\right) v,
$$

then

$$
\frac{\partial v_{1}}{\partial t}=\left(2 i k^{2}-i q(x, t) q^{*}(-x, t)\right) v_{1}+\left(-2 k q(x, t)-i q_{x}(x, t)\right) v_{2}
$$

and

$$
\frac{\partial v_{2}}{\partial t}=\left(2 k q^{*}(-x, t)+i q_{x}^{*}(-x, t)\right) v_{1}+\left(-2 i k^{2}+i q(x, t) q^{*}(-x, t)\right) v_{2} .
$$

Note that

$$
q(x, t) \rightarrow q_{ \pm}=q_{0} e^{2 i q_{0}^{2} t+i \theta_{ \pm}}, \text {as } x \rightarrow \pm \infty,
$$


where $q_{0}>0,0 \leq \theta_{ \pm}<2 \pi, \theta_{+}-\theta_{-}=\pi$. Thus,

$$
\frac{\partial v_{1}}{\partial t} \sim i\left(2 k^{2}+q_{0}^{2}\right) \cdot v_{1}-2 k q_{0} e^{2 i q_{0}^{2} t+i \theta_{+}} \cdot v_{2}
$$

and

$$
\frac{\partial v_{2}}{\partial t} \sim 2 k q_{0} e^{-2 i q_{0}^{2} t-i \theta_{-}} \cdot v_{1}-i\left(2 k^{2}+q_{0}^{2}\right) \cdot v_{2}
$$

as $x \rightarrow+\infty$, and

$$
\frac{\partial v_{1}}{\partial t} \sim i\left(2 k^{2}+q_{0}^{2}\right) \cdot v_{1}-2 k q_{0} e^{2 i q_{0}^{2} t+i \theta_{-}} \cdot v_{2}
$$

and

$$
\frac{\partial v_{2}}{\partial t} \sim 2 k q_{0} e^{-2 i q_{0}^{2} t-i \theta_{+}} \cdot v_{1}-i\left(2 k^{2}+q_{0}^{2}\right) \cdot v_{2}
$$

as $x \rightarrow-\infty$. As $x \rightarrow \pm \infty$, the eigenfunctions of the scattering problem asymptotically satisfy

$$
\left(\begin{array}{l}
v_{1} \\
v_{2}
\end{array}\right)_{x}=\left(\begin{array}{cc}
-i k & q_{0} e^{2 i q_{0}^{2} t+i \theta_{ \pm}} \\
-q_{0} e^{-2 i q_{0}^{2} t-i \theta_{\mp}} & i k
\end{array}\right)\left(\begin{array}{l}
v_{1} \\
v_{2}
\end{array}\right),
$$

we can get

$$
q_{0} e^{2 i q_{0}^{2} t+i \theta_{ \pm}} \cdot v_{2} \sim \frac{\partial v_{1}}{\partial x}+i k v_{1}
$$

and

$$
q_{0} e^{-2 i q_{0}^{2} t-i \theta_{\mp}} \cdot v_{1} \sim-\frac{\partial v_{2}}{\partial x}+i k v_{2}
$$

as $x \rightarrow \pm \infty$. Hence,

$$
\frac{\partial v_{1}}{\partial t} \sim i q_{0}^{2} \cdot v_{1}-2 k \frac{\partial v_{1}}{\partial x}
$$

and

as $x \rightarrow \pm \infty$.

$$
\frac{\partial v_{2}}{\partial t} \sim-i q_{0}^{2} \cdot v_{2}-2 k \frac{\partial v_{2}}{\partial x}
$$

Note that the eigenfunctions themselves, whose boundary values at space infinities, are not compatible with this time evolution. Therefore, one introduces time-dependent eigenfunctions

$$
\begin{aligned}
& \Phi(x, t)=e^{i A_{\infty} t} \cdot \phi(x, t), \quad \bar{\Phi}(x, t)=e^{i B_{\infty} t} \cdot \bar{\phi}(x, t), \\
& \Psi(x, t)=e^{i C_{\infty} t} \cdot \psi(x, t), \quad \bar{\Psi}(x, t)=e^{i D_{\infty} t} \cdot \bar{\psi}(x, t)
\end{aligned}
$$

to be solutions of (A21). We have

$$
\begin{aligned}
& \frac{\partial \Phi_{1}(x, t)}{\partial t}=i A_{\infty} \Phi_{1}(x, t)+e^{i A_{\infty} t} \frac{\partial \phi_{1}(x, t)}{\partial t}, \quad \frac{\partial \Phi_{2}(x, t)}{\partial t}=i A_{\infty} \Phi_{2}(x, t)+e^{i A_{\infty} t} \frac{\partial \phi_{2}(x, t)}{\partial t}, \\
& \frac{\partial \bar{\Phi}_{1}(x, t)}{\partial t}=i B_{\infty} \bar{\Phi}_{1}(x, t)+e^{i B_{\infty} t} \frac{\partial \bar{\phi}_{1}(x, t)}{\partial t}, \quad \frac{\partial \bar{\Phi}_{2}(x, t)}{\partial t}=i B_{\infty} \bar{\Phi}_{2}(x, t)+e^{i B_{\infty} t} \frac{\partial \bar{\phi}_{2}(x, t)}{\partial t}, \\
& \frac{\partial \Psi_{1}(x, t)}{\partial t}=i C_{\infty} \Psi_{1}(x, t)+e^{i C_{\infty} t} \frac{\partial \psi_{1}(x, t)}{\partial t}, \quad \frac{\partial \Psi_{2}(x, t)}{\partial t}=i C_{\infty} \Psi_{2}(x, t)+e^{i C_{\infty} t} \frac{\partial \psi_{2}(x, t)}{\partial t}, \\
& \frac{\partial \bar{\Psi}_{1}(x, t)}{\partial t}=i D_{\infty} \bar{\Psi}_{1}(x, t)+e^{i D_{\infty} t} \frac{\partial \bar{\psi}_{1}(x, t)}{\partial t}, \quad \frac{\partial \bar{\Psi}_{2}(x, t)}{\partial t}=i D_{\infty} \bar{\Psi}_{2}(x, t)+e^{i D_{\infty} t} \frac{\partial \bar{\psi}_{2}(x, t)}{\partial t} .
\end{aligned}
$$

Note that

$$
\phi(x, t) \sim\left(\begin{array}{c}
\lambda+k \\
-i q_{0} e^{-2 i q_{0}^{2} t-i \theta_{+}}
\end{array}\right) e^{-i \lambda x}, \quad \frac{\partial \phi(x, t)}{\partial t} \sim\left(\begin{array}{c}
0 \\
-2 q_{0}^{3} e^{-2 i q_{0}^{2} t-i \theta_{+}}
\end{array}\right) e^{-i \lambda x}
$$

as $x \rightarrow-\infty$. From

$$
\frac{\partial \Phi_{1}(x, t)}{\partial t} \sim i q_{0}^{2} \cdot \Phi_{1}(x, t)-2 k \frac{\partial \Phi_{1}(x, t)}{\partial x}=i A_{\infty} \Phi_{1}(x, t)+e^{i A_{\infty} t} \frac{\partial \phi_{1}(x, t)}{\partial t},
$$


as $x \rightarrow-\infty$, we can deduce

$$
A_{\infty}=q_{0}^{2}+2 \lambda k
$$

Similarly, we have

$$
\begin{gathered}
B_{\infty}=-A_{\infty}=-q_{0}^{2}-2 \lambda k, \\
C_{\infty}=-A_{\infty}=-q_{0}^{2}-2 \lambda k, \\
D_{\infty}=A_{\infty}=q_{0}^{2}+2 \lambda k .
\end{gathered}
$$

Then

$$
\begin{aligned}
& \frac{\partial \phi}{\partial t}=\left(\begin{array}{cc}
2 i k^{2}-i q(x, t) q^{*}(-x, t)-i\left(q_{0}^{2}+2 \lambda k\right) & -2 k q(x, t)-i q_{x}(x, t) \\
2 k q^{*}(-x, t)+i q_{x}^{*}(-x, t) & -2 i k^{2}+i q(x, t) q^{*}(-x, t)-i\left(q_{0}^{2}+2 \lambda k\right)
\end{array}\right) \phi \\
& \frac{\partial \bar{\phi}}{\partial t}=\left(\begin{array}{cc}
2 i k^{2}-i q(x, t) q^{*}(-x, t)+i\left(q_{0}^{2}+2 \lambda k\right) & -2 k q(x, t)-i q_{x}(x, t) \\
2 k q^{*}(-x, t)+i q_{x}^{*}(-x, t) & -2 i k^{2}+i q(x, t) q^{*}(-x, t)+i\left(q_{0}^{2}+2 \lambda k\right)
\end{array}\right) \bar{\phi} \\
& \frac{\partial \psi}{\partial t}=\left(\begin{array}{cc}
2 i k^{2}-i q(x, t) q^{*}(-x, t)+i\left(q_{0}^{2}+2 \lambda k\right) & -2 k q(x, t)-i q_{x}(x, t) \\
2 k q^{*}(-x, t)+i q_{x}^{*}(-x, t) & -2 i k^{2}+i q(x, t) q^{*}(-x, t)+i\left(q_{0}^{2}+2 \lambda k\right)
\end{array}\right) \psi, \\
& \frac{\partial \bar{\psi}}{\partial t}=\left(\begin{array}{cc}
2 i k^{2}-i q(x, t) q^{*}(-x, t)-i\left(q_{0}^{2}+2 \lambda k\right) & -2 k q(x, t)-i q_{x}(x, t) \\
2 k q^{*}(-x, t)+i q_{x}^{*}(-x, t) & -2 i k^{2}+i q(x, t) q^{*}(-x, t)-i\left(q_{0}^{2}+2 \lambda k\right)
\end{array}\right) \bar{\psi} .
\end{aligned}
$$

Note that

$$
\phi(x, t)=b(t) \psi(x, t)+a(t) \bar{\psi}(x, t)
$$

and

$$
\bar{\phi}(x, t)=\bar{a}(t) \psi(x, t)+\bar{b}(t) \bar{\psi}(x, t),
$$

then

$$
\begin{aligned}
& \left(\begin{array}{cc}
2 i k^{2}-i q(x, t) q^{*}(-x, t)-i\left(q_{0}^{2}+2 \lambda k\right) & -2 k q(x, t)-i q_{x}(x, t) \\
2 k q^{*}(-x, t)+i q_{x}^{*}(-x, t) & -2 i k^{2}+i q(x, t) q^{*}(-x, t)-i\left(q_{0}^{2}+2 \lambda k\right)
\end{array}\right) \\
& \cdot\left(\begin{array}{l}
b(t) \psi_{1}(x, t)+a(t) \bar{\psi}_{1}(x, t) \\
b(t) \psi_{2}(x, t)+a(t) \bar{\psi}_{2}(x, t)
\end{array}\right)=\frac{\partial b(t)}{\partial t} \cdot\left(\begin{array}{l}
\psi_{1}(x, t) \\
\psi_{2}(x, t)
\end{array}\right) \\
& +b(t)\left(\begin{array}{cc}
2 i k^{2}-i q(x, t) q^{*}(-x, t)+i\left(q_{0}^{2}+2 \lambda k\right) & -2 k q(x, t)-i q_{x}(x, t) \\
2 k q^{*}(-x, t)+i q_{x}^{*}(-x, t) & -2 i k^{2}+i q(x, t) q^{*}(-x, t)+i\left(q_{0}^{2}+2 \lambda k\right)
\end{array}\right)\left(\begin{array}{l}
\psi_{1}(x, t) \\
\psi_{2}(x, t)
\end{array}\right) \\
& +\frac{\partial a(t)}{\partial t} \cdot\left(\begin{array}{l}
\bar{\psi}_{1}(x, t) \\
\bar{\psi}_{2}(x, t)
\end{array}\right)+a(t) \\
& \cdot\left(\begin{array}{cc}
2 i k^{2}-i q(x, t) q^{*}(-x, t)-i\left(q_{0}^{2}+2 \lambda k\right) & -2 k q(x, t)-i q_{x}(x, t) \\
2 k q^{*}(-x, t)+i q_{x}^{*}(-x, t) & -2 i k^{2}+i q(x, t) q^{*}(-x, t)-i\left(q_{0}^{2}+2 \lambda k\right)
\end{array}\right)\left(\begin{array}{c}
\bar{\psi}_{1}(x, t) \\
\bar{\psi}_{2}(x, t)
\end{array}\right),
\end{aligned}
$$

i.e.,

$$
\frac{\partial b(t)}{\partial t} \cdot\left(\begin{array}{l}
\psi_{1}(x, t) \\
\psi_{2}(x, t)
\end{array}\right)+b(t)\left(\begin{array}{cc}
2 i A_{\infty} & 0 \\
0 & 2 i A_{\infty}
\end{array}\right)\left(\begin{array}{l}
\psi_{1}(x, t) \\
\psi_{2}(x, t)
\end{array}\right)+\frac{\partial a(t)}{\partial t}\left(\begin{array}{l}
\bar{\psi}_{1}(x, t) \\
\bar{\psi}_{2}(x, t)
\end{array}\right)=0 .
$$

Taking $x \rightarrow+\infty$, since $\psi(x, t)$ and $\bar{\psi}(x, t)$ are linearly independent, so

$$
\frac{\partial a(t)}{\partial t}=0, \quad \frac{\partial b(t)}{\partial t}=-2 i A_{\infty} b(t) .
$$

Similarly, we can get

$$
\frac{\partial \bar{a}(t)}{\partial t}=0, \quad \frac{\partial \bar{b}(t)}{\partial t}=2 i A_{\infty} \bar{b}(t)
$$

Therefore, both $a(t)$ and $\bar{a}(t)$ are time independent, and

$$
b\left(i q_{0}, t\right)=b\left(i q_{0}, 0\right) e^{-2 i\left(q_{0}^{2}+2 \lambda k\right) t}=e^{i \theta_{1}} \cdot e^{-2 i q_{0}^{2} t}
$$


and

$$
\bar{b}\left(-i q_{0}, t\right)=\bar{b}\left(-i q_{0}, 0\right) e^{2 i\left(q_{0}^{2}+2 \lambda k\right) t}=e^{i \bar{\theta}_{1}} \cdot e^{2 i q_{0}^{2} t}=e^{i\left(\theta_{1}+2 \theta_{+}\right)} \cdot e^{2 i q_{0}^{2} t} .
$$

${ }^{1}$ Ablowitz, M. J. and Musslimani, Z. H., “Integrable nonlocal nonlinear Schrödinger equation,” Phys. Rev. Lett. 110, 064105 (2013).

${ }^{2}$ Ablowitz, M. J. and Musslimani, Z. H., "Integrable discrete PT symmetric model,” Phys. Rev. E 90, 032912 (2014).

${ }^{3}$ Ablowitz, M. J. and Musslimani, Z. H., "Inverse scattering transform for the integrable nonlocal nonlinear Schrödinger equation," Nonlinearity 29(3), 915 (2016).

${ }^{4}$ Ablowitz, M. J. and Musslimani, Z. H., "Integrable nonlocal nonlinear equations," Stud. Appl. Math. 139(1), 7-59 (2017).

${ }^{5}$ Ablowitz, M. J. and Clarkson, P. A., Solitons, Nonlinear Evolution Equations and Inverse Scattering (Cambridge University Press, Cambridge, 1991).

${ }^{6}$ Ablowitz, M. J., Kaup, D. J., Newell, A. C., and Segur, H., "The inverse scattering transform-Fourier analysis for nonlinear problems," Stud. Appl. Math. 53, 249-315 (1974).

${ }^{7}$ Ablowitz, M. J. and Segur, H., Solitons and Inverse Scattering Transform, SIAM Studies in Applied Mathematics Vol. 4 (SIAM, Philadelphia, PA, 1981).

${ }^{8}$ Ablowitz, M. J., Nonlinear Dispersive Waves: Asymptotic Analysis and Solitons (Cambridge University Press, Cambridge, 2011).

${ }^{9}$ Ablowitz, M. J., Prinari, B., and Trubatch, A. D., Discrete and Continuous Nonlinear Schrödinger Systems (Cambridge University Press, Cambridge, 2004).

${ }^{10}$ Biondini, G. and Kovacic, G., "Inverse scattering transform for the focusing nonlinear Schrödinger equation with nonzero boundary conditions," J. Math. Phys. 55, 031506 (2014).

${ }^{11}$ Demontis, F., Prinari, B., van der Mee, C., and Vitale, F., "The inverse scattering transform for the defocusing nonlinear Schrödinger equations with nonzero boundary conditions," Stud. Appl. Math. 131, 1-40 (2012).

${ }^{12}$ Demontis, F., Prinari, B., van der Mee, C., and Vitale, F., "The inverse scattering transform for the focusing nonlinear Schrödinger equations with asymmetric boundary conditions," J. Math. Phys. 55, 101505 (2014).

${ }^{13}$ Faddeev, L. D. and Takhtajan, L. A., Hamiltonian Methods in the Theory of Solitons (Springer, Berlin, 1987).

${ }^{14}$ Gadzhimuradov, T. A. and Agalarov, A. M., "Towards a gauge-equivalent magnetic structure of the nonlocal nonlinear Schrödinger equation,” Phys. Rev. A 93, 062124 (2016).

${ }^{15} \mathrm{He}$, J. S. and Qiu, D. Q., "Mirror symmetrical nonlocality of a parity-time symmetry system," private communication (2016).

${ }^{16}$ Kivshar, Y. and Agrawal, G. P., Optical Solitons: From Fibers to Photonic Crystals (Academic Press, 2003).

${ }^{17}$ Konotop, V. V., Yang, J., and Zezyulin, D. A., "Nonlinear waves in PT-symmetric systems," Rev. Mod. Phys. 88, 035002 (2016).

${ }^{18}$ Korteweg, D. J. and de Vries, G., "On the change of form of long waves advancing in a rectangular canal, and on a new type of long stationary waves," Philos. Mag. Ser. 5 39, 422 (1895).

${ }^{19}$ Prinari, B., Ablowitz, M. J., and Biondini, G., "Inverse scattering transform for the vector nonlinear Schrödinger equation with nonvanishing boundary conditions," J. Math. Phys. 47, 063508 (2006).

${ }^{20}$ Ruter, C. E., Makris, K. G., El-Ganainy, R., Christodoulides, D. N., Segev, M., and Kip, D., "Observation of parity time symmetry in optics," Nat. Phys. 6, 192 (2010).

${ }^{21}$ Segev, M., "Optical spatial solitons," Opt. Quantum Electron. 30, 503-533 (1998).

${ }^{22}$ Zakharov, V. E. and Shabat, A. B., "Exact theory of two-dimensional self-focusing and one-dimensional self-modulation of waves in nonlinear media," Sov. Phys. JETP 34, 63-69 (1972).

${ }^{23}$ Zakharov, V. E. and Shabat, A. B., "Interaction between solitons in a stable medium," Sov. Phys. JETP 37, 823-828 (1973),

${ }^{24}$ Zverovich, E. I., "Boundary value problems in the theory of analytic functions in Hölder classes on Riemann surfaces," Russ. Math. Surv. 26(1), 117 (1971). 